\title{
Synopsis of the
}

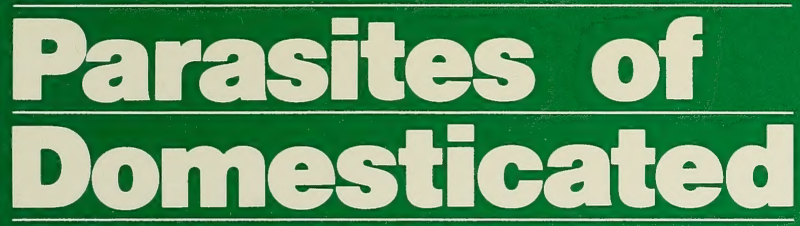

of Canada

\section{Murray J. Kennedy}


Other titles in this series -

Synopsis of the Parasites of Vertebrates of Canada - Helminths and Protozoa of Terrestrial Mammals Murray J. Kennedy, 1986

Synopsis of the Parasites of Vertebrates of Canada - Ectoparasites of Terrestrial Mammals

Murray J. Kennedy and Robert A. Newman, 1986.

Synopsis of the Digenea of Mammals of North America Murray J. Kennedy, 1988.

Copies of these publications are available from the Queen's Printer, 11510 Kingsway Avenue, Edmonton T5G 2 Y5 Each order must be accompanied by cheque for $\$ 5.00$ made out to The Provincial Treasurer 


\section{Synopsis of the}
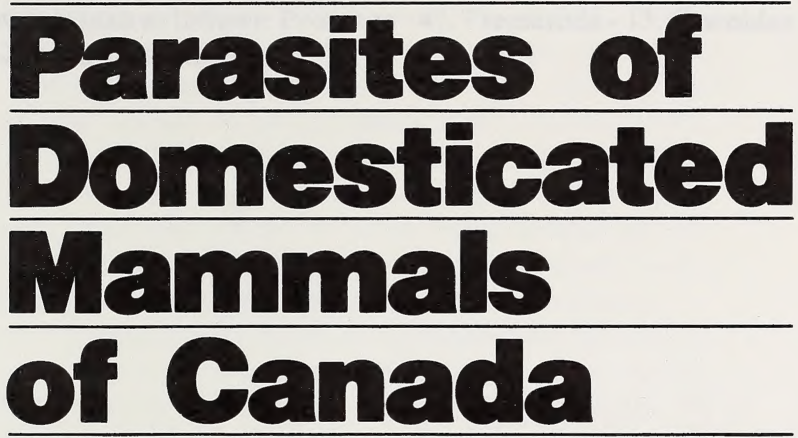

Murray J. Kennedy

Alberta Agriculture

Veterinary Laboratory

6909 - 116 Street,

Edmonton, Alberta

Canada

T6H 4P2 


\begin{abstract}
The revised edition of this synopsis contains Parasite-Host and Host-Parasite lists of 247 species of parasites from seven domesticated mammals. Four species, the goat (Capra hircus), ox (Bos taurus), pig (Sus scrofa), and sheep (Ovis aries) belong in the Artiodactyla; one species, the horse (Equus caballus) in the Perissodactyla; and two species, the cat (Felis catus) and dog (Canis familiaris) belong in the Carnivora. The 247 species of parasites are distributed among higher taxa as follows: Protozoa -47; Trematoda - 13; Cestoidea -22; Nematoda - 109; Acanthocephala - 2; Chelicerata -21 ; and Uniramia - 33 .
\end{abstract}

\title{
Preface to the Second Edition
}

About two years have elapsed since the publication of the first edition of this synopsis. During this interval I have tried to gather pertinent references and literature necessary for the publication of this revised edition. The major effort involved in this revision has been directed toward updating and improving the accuracy and coverage of the material presented. As a result, 76 titles in technical journals was gathered and added to the original 185 titles. Additional papers were reviewed, and many placed in the list for supplementary reading. Colleagues have been generous in sending me reprints of their work and pointing out ommissions in the first edition. The general organization and approach utilized in the first edition has been retained in the revised edition. New entries have been made for most groups, especially the Protozoa and Chelicerata. The recent trend to omit specific names for the cysticerci of the various Taenia spp. has been adopted. 


\section{Introduction}

The compiled information covers the period from 1877 to December 1987 and was extracted from numerous publications in the primary scientific literature. Journal series such as the Canadian Journal of Zoology, Canadian Journal of Research, Canadian Veterinary Journal, Journal of Protozoology, Proceeding of the Helminthological Society of Washington, and the Journal of Parasitology were searched in their entirety. Abstracting series such as Zoological Records, Helminthological Abstracts, Protozoology Abstracts, and Veterinary Bulletin were scanned for additional references. Theses and reports of federal and provincial governments and of other institutions were not searched extensively, hence, some existing records may have been omitted.

In providing references to the parasite-host-distribution data, I have included only reports based on original records, but screening all reports to ensure complete exclusion of repetitive records based on the same material was not possible. Publications that do not provide original parasite-hostdistribution records but deal with some aspect of Canadian veterinary parasitology are compiled in a supplementary list of references.

I conducted a least squares regression analysis on the number of papers published since 1877 (Figure 1) to estimate the number of publications we may expect to see in the 1980 's. The estimate was based on the multivariate general linear model: $\mathrm{y}=\mathrm{mx}+\mathrm{b}$ where $\mathrm{y}$ is a vector of dependent variables, $\mathrm{x}$ is a vector of independent variables, $\mathrm{b}$ is a vector of regression coefficients, and $\mathrm{m}$ is a vector of random errors. The analysis predicted 96 papers will be published in the 1980 's. In the present synopsis 64 papers up to December 1987 were reviewed.
The synopsis indicates that up to December, 1987, at least 247 species of parasites have been reported from seven species of domesticated mammals. Parasites not identified to the species level are not included in the total. The 247 species are divided among major taxa as follows: Protozoa - 47; Trematoda - 13; Cestoidea - 22; Nematoda - 109; Acanthocephala - 2, Chelicereta - 21; and Uniramia - 33.

The taxonomic arrangement adopted for each phylum or class of parasitic organism included in Canadian records, in most cases, is as follows:

Protozoa - Levine et al. (1980)

Trematoda - Brooks et al. (1985)

Cestoidea - Schmidt (1986)

Nematoda - Anderson et al. (1974-1983)

Acanthocephala - Amin (1982)

Acarina - Krantz (1978)

Diptera - Arnett et al. (1980)

Mallophaga - Emerson (1972)

Siphonaptera - Holland (1984)

Siphunculata - Strojanovich and Pratt (1965)

Host nomenclature and classification conforms to that of Nowak and Paradiso (1983). The entries in the synopsis are organized in accordance with the format used by Margolis and Arthur (1979) in their Synopsis of the parasites of fishes of Canada.

The Parasite-Host List is organized by higher taxa of parasites in the following order: Protozoa, Platyhelminthes (Trematoda, Cestoidea), Nematoda, Acanthocephala, Chelicerata and Uniramia. Species, genera, and family names are listed in alphabetical order within their respective next higher

Figure 1 Number of papers published on the parasites of domesticated mammals from 1877 to December, 1987.

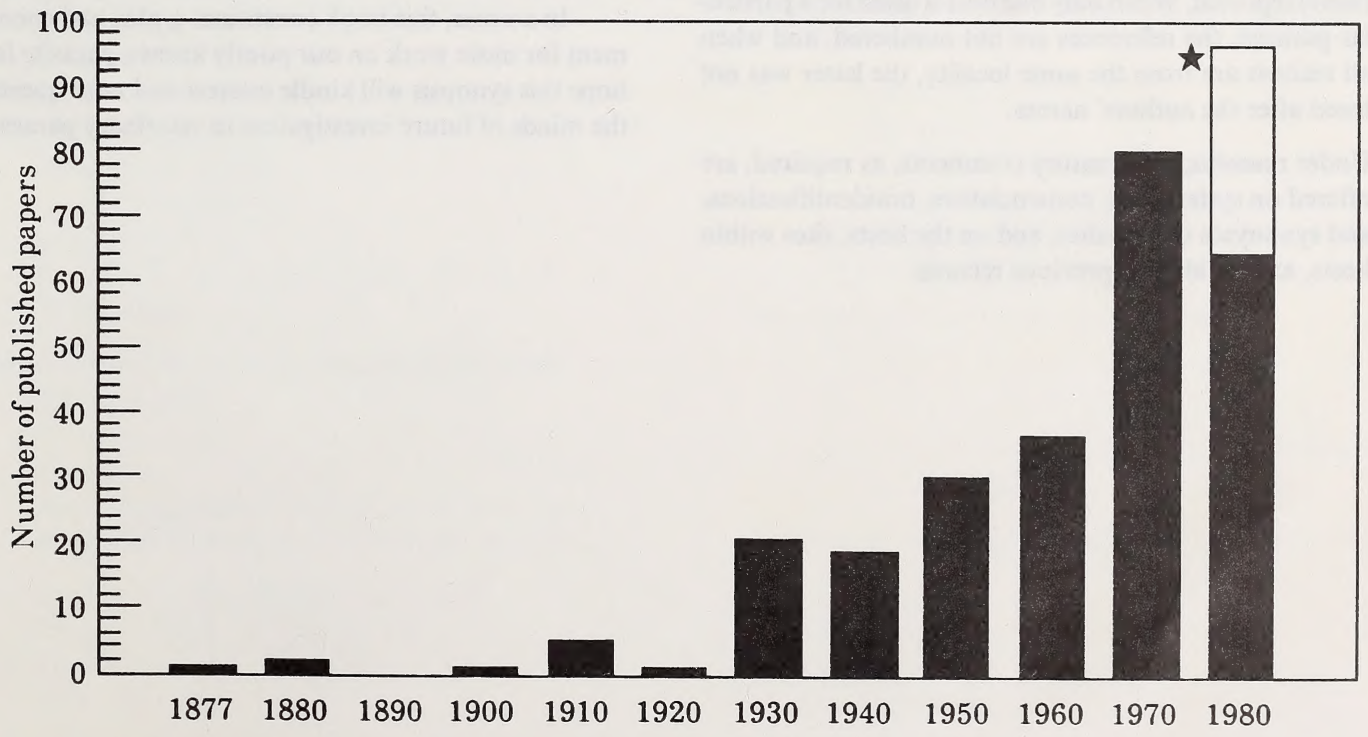

$\star$ Predicted number of papers published by the end of 1989 
taxon, which in the case of a family is either a superfamily or an order, depending on the group in question and the usefulness of including the superfamily or order category.

For each parasite species listed, the following information is provided:

1) The current scientific name, including author(s) and date(s), followed by any recognized synonyms that may have been used in establishing the Canadian record(s). No attempt has been made to evaluate systematically the validity of published records, but attention is drawn to obvious errors. Adult and larval stages are recorded separately for the Cestoidea and Nematoda.

2) The site of occurrence of the parasite in its host(s) (aberrant sites usually are not included). When the site was not given, the likely site was determined from other records, and is placed in square brackets.

3) The hosts. Numbers in parentheses after each host name correspond with the numbers assigned to the references establishing the particular parasite-host records.

4) Distribution (Dist.) Species distribution is indicated by Provincial or Territory boundaries listed in alphabetical order as follows: Alta (Alberta), BC (British Columbia), Man (Manitoba), NB (New Brunswick), Nfld (Newfoundland, includes Labrador), NS (Nova Scotia), NWT (Northwest Territories), Ont (Ontario), PEI (Prince Edward Island), Que (Quebec), Sask (Saskatchewan), and YT (Yukon Territory).

5) Authors responsible for the published records are listed in chronological order. To enable the reader to grasp at a glance the author(s) responsible for particular parasitehost-locality records the references are numbered and listed under "Records" and the corresponding number(s) placed after the host name. Each reference is followed by the locality or localities from which the parasite was (were) reported. When only one host is listed for a particular parasite, the references are not numbered, and when all records are from the same locality, the latter was not listed after the authors' names.

6) Under remarks, explanatory comments, as required, are offered on systematics, nomenclature, misidentifications, and synonyms of parasites; and on the hosts, sites within hosts, and validity of previous records.
In the Host-Parasite List, within each order, the families, genera, and species are listed alphabetically. After the name of each parasite species, its geographic distribution in the host in question is given in parentheses.

The REFERENCES are given in two parts, the first includes those directly referred to in the text, and the second, SUPPLEMENTARY REFERENCES, contains a list of publications dealing with various aspects of parasites of Canadian domestic mammals but which do not contain original parasite-host records.

To facilitate access to information in the parasite-host and host-parasite lists, the publication concludes with an INDEX to parasite and host scientific names.

There is no comprehensive summary of the parasites of dometicated mammals of Canada. Therefore, the need for the present volume was obvious. I hope this synopsis will prove useful to biologists and zoologists generally, and to practitioners and veterinary parasitologists particularly. My role as author was one of selection, arrangement and organization of a large mass of published information. I have tried to be complete, accurate, concise and consistent from one section to another. I tried to keep each section up to date in accordance with the latest classification schemes. For the most part, only parasitic organisms are included. Commensals, micropredators, pests, phoretic mites, etc. are not included.

The list of references at the end include older classical papers as well as recent contributions. Papers pertinent to parasites of domesticated mammals, but do not themselves constitute new records from Canada, are referenced in a section on supplementary reading.

Obviously a book of this sort is bound to have errors and ambiguities, and certainly such faults are my responsibility. I hope readers will bring them to my attention. I also hope that colleagues will send me reprints of the current work or papers I missed so that these additions or corrections can be included in future revisions of the synopsis.

In a sense, this book constitutes a plea and encouragement for more work on our poorly known parasite fauna. I hope this synopsis will kindle interest and raise questions in the minds of future investigators in veterinary parasitology. 


\section{PARASITE-HOST LIST}


Digitized by the Internet Archive in 2016

https://archive.org/details/synopsisofparasi00kenn_2 


\section{KINGDOM ANIMALIA \\ SUBKINGDOM PROTOZOA}

\section{PHYLUM APICOMPLEXA}

\section{CLASS SPOROZOEA}

ORDER EUCOCCIDIIDA

SUBORDER ADELEINA

Klossiella equi Baumann, 1946

Site: kidney

Host: Equus caballus

Dist.: NB

Record: Austin and Dies 1981

SUBORDER EIMERIINA

Cryptosporidium spp.

Site: intestine

Host: Bos taurus $(1,3,4)$

\section{Equus caballus (2)}

Sus scrofa (5)

Dist.: Man, Ont, Sask

Record: 1. Sanford and Josephson 1982 (Ont);

2. Gajadhar et al. 1985 (Sask); 3. Mann et al.

1986 (Man); 4. Mann et al. 1987 (Man); 5.

Sanford 1987 (Ont)

Eimeria ahsata Honess, 1942

Site: [intestine]

Host: Ovis aries

Dist.: Alta, Sask

Records: Mahrt 1969 (Alta); Horton and

Stockdale 1981 (Sask)

Eimeria alabamensis Christensen, 1941

Site: [intestine]

Host: Bos taurus

Dist.: Alta, Ont

Record: Slocombe 1973a (Ont); Kennedy and

Kralka 1987 (Alta)

Eimeria arloingi (Marotel, 1905) Martin, 1909

Site: [intestine]

Hosts: Bos taurus (2)

Ovis aries $(1,2)$

Dist.: Alta, Ont

Records: 1. Mahrt 1969 (Alta); 2. Slocombe 1973a (Ont)

Eimeria aubernensis Christensen and Porter, 1939

Site: [intestine]

Host: Bos taurus

Dist.: Alta, Ont

Records: Slocombe 1973a (Ont); Kennedy and

Kralka 1987 (Alta); Reid and Madill 1987 (Ont)

Eimeria bovis (Zublin, 1908) Fiebiger, 1912

Site: [intestine]

Host: Bos taurus

Dist.: Alta, Ont
Records: Slocombe 1973a (Ont); Kennedy and Kralka 1987 (Alta)

Eimeria bukidnonensis Tubangui, 1931

Site: [intestine]

Host: Bos taurus

Dist.: Ont

Record: Slocombe 1973a

Eimeria canadensis Bruce, 1921

Site: [intestine]

Host: Bos taurus

Dist.: Alta, Ont

Records: Slocombe 1973a (Ont); Kennedy and

Kralka 1987 (Alta)

Eimeria canis Wenyon, 1923

Site: [intestine]

Host:Canis familiaris

Dist.: Ont, Que

Records: Choquette and Gelinas 1950 (Que);

Slocombe 1973a (Ont)

Eimeria crandallis Honess, 1942

Site: [intestine]

Hosts: Equus caballus (2)

Ovis aries (1)

Dist.: Alta, Ont

Records: 1. Mahrt 1969 (Alta); 2. Slocombe 1973a (Ont)

Eimeria cylindrica Wilson, 1931

Site: [intestine]

Host: Bos taurus

Dist.: Alta, Ont

Records: Slocombe 1973a (Ont); Kennedy and

Kralka 1987 (Alta)

Eimeria debliecki Douwes, 1921

Site: [intestine]

Host: Sus scrofa

Dist.: Ont, Que

Records: Slocombe 1973a (Ont); Robinson and Morin 1982 (Que)

Eimeria ellipsoidalis Becker and Frye, 1929

Site: [intestine]

Host: Bos taurus

Dist.: Alta, Ont

Records: Slocombe 1973a (Ont); Kennedy and Kralka 1987 (Alta)

Eimeria faurei (Moussu and Marotel, 1902) Martin, 1909

Site: [intestine]

Host: Ovis aries

Dist.: Alta

Record: Mahrt 1969 
Eimeria granulosa Christensen, 1938

Site: [intestine]

Hosts: Equus caballus (2) Ovis aries (1)

Dist.: Alta, Ont

Records: 1. Mahrt 1969 (Alta); 2. Slocombe 1973a (Ont)

Eimeria illinoisensis Levine and Ivens, 1967

Site: [intestine]

Host: Bos taurus

Dist.: Alta

Record: Kennedy and Kralka 1987 (Alta)

Eimeria intricata Spiegl, 1925

Site: [intestine]

Hosts: Equus caballus (2)

Ovis aries (1)

Dist.: Alta, Ont

Records: 1. Mahrt 1969 (Alta); 2. Slocombe 1973a (Ont)

Eimeria leuckarti (Flesch, 1883) Reichenow, 1940

Site: [intestine]

Host: Equus caballus

Dist.: Ont

Record: Slocombe 1973a

Eimeria ninakohlyakimovae Yakimoff and

Site: [intestine]

Rastegaieff, 1930

Host: Ovis aries

Dist.: Alta, Ont, Sask

Records: Mahrt 1969 (Alta); Slocombe 1973a

(Ont); Horton and Stockdale 1981 (Sask)

Eimeria ovina Levine and Ivens, 1970

Site: [intestine]

Host: Ovis aries

Dist.: Sask

Record: Horton and Stockdale 1981

Eimeria pallida Christensen, 1938

Site: [intestine]

Host: Ovis aries

Dist.: Alta

Record: Mahrt 1969

Eimeria parva Kotlan, Mocsy, and Vajda, 1929

Site: [intestine]

Host: Ovis aries

Dist.: Alta, Ont, Sask

Records: Mahrt 1969 (Alta); Slocombe 1973a

(Ont); Horton and Stockdale 1981 (Sask)

Eimeria punctata Landers, 1955

Site: [intestine]

Host:Ovis aries

Dist.: Alta

Record: Mahrt 1969

Eimeria wyomingensis Huizinga and Winger, 1942

Site: [intestine]

Host: Bos taurus

Dist.: Alta, Ont

Records: Slocombe 1973a (Ont); Kennedy and

Kralka 1987 (Alta)
Eimeria zuernii (Rivolta, 1878) Martin, 1909

Site: [intestine]

Host: Bos taurus

Dist.: Alta, Ont

Records: Slocombe 1973a (Ont); Kennedy and

Kralka 1987 (Alta)

Eimeria spp.

Site: [intestine]

Hosts: Bos taurus (2)

Ovis aries (1)

Dist.: Alta, Que

Record: 1. Swales 1940b (Que); 2. Kennedy and Kralka 1987 (Alta)

Isospora bigemina (Stiles, 1891) Luhe, 1906

Site: intestine

Host: Canis familiaris

Dist.: Que, Sask

Records: Choquette and Gelinas 1950 (Que);

Olson 1985 (Sask)

Isospora canis Nemeseri, 1959

Site: [intestine]

Host: Canis familiaris

Dist.: Sask

Record: Olson 1985

Isospora felis Wenyon, 1926

Site: [intestine]

Hosts: Canis familiaris $(1,2)$

Felis catus (2)

Dist.: Ont, Que

Records: 1. Choquette and Gelinas 1950 (Que);

2. Slocombe 1973a (Ont)

Isospora ohioensis Dubey, 1975

Site: [intestine]

Host: Canis familiaris

Dist.: Sask

Record: Olson 1985

Isospora rivolta (Grassi, 1879) Wenyon, 1923

Site: [intestine]

Hosts: Canis familiaris $(1,2)$

Felis catus (2)

Dist.: Ont, Que

Records: 1. Choquette and Gelinas 1950 (Que);

2. Slocombe 1973a (Ont)

Isospora suis Biester in Becker, 1934

Site: [intestine]

Host: Sus scrofa

Dist.: Ont, Que

Records: Sanford and Josephson 1981 (Ont);

Robinson and Morin 1982 (Que); Morin et al.

1983 (Que)

Isospora wallacei Dubey, 1976

Site: [intestine]

Host: Canis familiaris

Dist.: Sask

Record: Olson 1985

Remarks: Levine and Ivens (1986) consider this species a synonym of Toxoplasma bahiensis (de Moura Costa, 1956) Levine, 1983. 
Isospora spp.

Site: [intestine]

Hosts: Canis familiaris $(1,2,3,4,5)$ Felis catus (5)

Dist.: Alta, NB, NWT, Que, Sask

Records: 1. Saunders 1949 (Alta); 2. Unruh et al. 1973 (Alta, NWT, Sask); 3. Anvik et al. 1974

(Sask); 4. Seah et al. 1975 (Que); 5. Malloy and Embil 1978 (NB)

Remarks: Saunders (1949) believed his species was probably I. bigemina.

Sarcocystis cruzi (Hasselmann, 1923) Wenyon, 1926

Site: [muscle]

Host: Bos taurus

Dist.: Sask

Record: Gajadhar et al. 1987

Sarcocystis hirsuta Moule, 1888

Site: [muscle]

Host: Bos taurus

Dist.: Sask

Record: Gajadhar et al. 1987

Sarcocystis spp.

Site: heart

Hosts: Bos taurus $(1,2,4)$

Equus caballus (1)

Ovis aries (3)

Dist.: Ont, Sask

Records: 1. Slocombe 1973a (Ont); 2. Meads 1976 (Ont.); 3. Moteane et al. 1979 (Sask); 4.

Gajadhar et al. 1987 (Sask)

Toxoplasma gondii Nicolle and Manceaux, 1908

Site: unspecified

Hosts: Bos taurus (5)

Canis familiaris $(1,2,5)$

Capra hircus (4)

Equus caballus (5)

Felis catus $(1,3,5)$

Ovis aries (5)

Sus scrofa (5)

Dist.: Ont, Que, Sask

Record: 1. Hulland 1956(Ont); 2. Martin and Letarte 1977 (Que); 3. Nation and Allen 1976 (Sask); 4. Tizard et al. 1977 (Ont); 5. Tizard et al. 1978 (Ont)

Toxoplasma spp.

Site: brain, spinal cord, blood

Hosts: Bos taurus (3)

Canis familiaris (2)

Equus caballus $(2,4)$

Felis catus $(1,2,3)$

Ovis aries $(2,3)$

Sus scrofa (2)

Dist.: Ont, Que, Sask

Records: 1. Beauregard et al. 1965 (Que.); 2.

Slocombe 1973a (Ont); 3. Pechere et al. 1977

(Que); 4. Clark et al. 1981 (Sask)

Remarks: Pechere et al. 1977 (Que) found antitoxoplasmic antibodies by passive haemagglutination testing.
Unidentified Eucoccidiida

Site: [intestine]

Hosts: Bos taurus (2) Ovis aries $(1,3)$

Sus scrofa (4)

Dist.: Ont, Que, Sask

Records: 1. Griffiths 1937 (Que); 2. Slocombe 1973a (Ont); 3. Monteane et al. 1979 (Sask); 4. Morin et al. 1980 (Que)

PHYLUM CILIOPHORA

CLASS KINETOFRAGMINOPHOREA

ORDER TRICHOSTOMATIDA

Balantidium spp.

Site: [intestine]

Host: Sus scrofa

Dist.: Ont

Record: Slocombe 1973a

\section{PHYLUM SARCOMASTIGOPHORA}

SUBPHYLUM MASTIGOPHORA

CLASS ZOOMASTIGOPHOREA

ORDER DIPLOMONADIDA

\section{FAMILY HEXAMITIDAE}

Giardia canis Hegner, 1922

Site: [intestine]

Host: Canis familiaris

Dist.: Que, Sask

Records: Choquette and Gelinas 1950 (Que);

Olson 1985 (Sask)

Giardia spp.

Site: [intestine]

Hosts: Canis familiaris $(1,3,4)$ Bos taurus (2)

Dist.: Alta, NS, Ont, Sask

Records: 1. Slocombe 1973a (Ont); 2. Willson 1982 (Sask); 3. Cribb and Spracklin 1986 (NS);

4. Wallis et al. 1986 (Alta)

\section{ORDER KINETOPLASTIDA}

Trypanosoma equiperdum Doflein, 1901

Site: blood

Host: Equus caballus

Dist.: Alta

Record: Watson and Hadwen 1912

Trypanosoma rutherfordi Watson and Hadwen, 1912

Site: blood

Host: Bos taurus

Dist.: BC

Record: Watson and Hadwen 1912

Trypanosoma theileri Laveran, 1902

Site: blood

Host: Bos taurus

Dist.: Ont

Record: Woo et al. 1970; Julian 1971

Remarks: Julian (1971) recovered T. theileri-

like flagellates 
Trypanosoma uniforme Bruce et al., 1911

Site: blood

Host: Bos taurus

Dist.: Ont

Record: Woo et al. 1970

Remarks: Woo et al. (1970) stated only that the

Trypanosome resembled T. uniforme.

Trypanosoma spp.

Site: blood

Host: Bos taurus

Dist.: BC

Record: Watson and Hadwen 1912

\section{ORDER TRICHOMONADIDA}

\section{Trichomonas spp.}

Site: intestine

Hosts: Bos taurus (2)

Canis familiaris (1)

Equus caballus (2)

Sus scrofa (2)

Dist.: Ont

Records: 1. Comfort and Axelson 1962; 2.

Slocombe 1973a

\section{SUBPHYLUM SARCODINA}

\section{CLASS LOBOSEA}

ORDER AMOEBIDA

Endolimax nana (Wenyon and O'Conner, 1917)

Site: [intestine]

$$
\text { Brug, } 1918
$$

Host: Canis familiaris
Dist.: Sask

Record: Unruh et al. 1973

Entamoeba blattae

Site: [intestine]

Host: Canis familiaris

Dist.: Que

Record: Choquette and Gelinas 1950

Remarks: Choquette and Gelinas (1950)

considered $C$. familiaris an unnatural host for $E$. blattae. This protozoan is normally a parasite of cockroaches and termites.

Entamoeba coli (Grassi, 1879) Casagrandi and Barbagallo, 1895

Site: [intestine]

Host: Canis familiaris

Dist.: Que, Sask

Records: Unruh et al. 1973 (Sask); Seah et al. 1975 (Que)

Entamoeba histolytica Schaudinn, 1903

Site: [intestine]

Host: Canis familiaris

Dist.: Que, Sask

Records: Unruh et al. 1973 (Sask); Wittnich 1976 (Que) 


\section{SUBKINGDOM EUMETAZOA}

\section{PHYLUM PLATYHELMINTHES}

\section{CLASS TREMATODA}

SUBCLASS DIGENEA

FAMILY DICROCOELIIDAE

Dicrocoelium dendriticum (Rudolphi, 1819)

Looss, 1899

Syn.: Dicrocoelium lanceolatum Stiles and

Hassal, 1898

Site: bile ducts

Hosts: Bos taurus $(5,6)$

Ovis aries $(1,2,3,4)$

Dist.: BC, Nfld, NS, PEI, Que

Records: 1. Conklin and Baker 1930 (NS, PEI, Que); 2. Swales 1933a (Que), 3. 1940a (NS); 4.

Smith and Archibald 1965 (NS); 5. Smith and

Threlfall 1973 (Nfld); 6. Lewis 1974 (BC)

\section{FAMILY DIPLOSTOMIDAE}

Alaria arisaemoides Augustine and Uribe, 1927

Site: intestine

Host: Canis familiaris

Dist.: Sask

Record: Allen and Mills 1971

Alaria canis LaRue and Fallis, 1934

Site: [intestine]

Host: Canis familiaris

Dist.: Ont

Record: LaRue and Fallis 1934

Alaria spp.

Site: [intestine]

Host: Canis familiaris

Dist.: Alta, NWT, Ont, Que, Sask

Records: 1. Cameron et al. 1940 (NWT, Ont, Que, Sask); 2. Allen and Mills 1971 (Sask); 3. Unruh et al. 1973 (Alta, NWT, Sask); 4. Anvik et al. 1974 (Sask)

\section{FAMILY FASCIOLIDAE}

Fasciola hepatica Linnaeus, 1758

Site: [liver]

Hosts: Bos taurus (3) Ovis aries $(1,2)$

Dist.: BC, Canada, NB, Que

Record: 1. Griffiths 1939 (BC, NB, Que); 2.

Swales 1940a (Canada); 3. Bouvry and Rau 1986 (Que)

Fascioloides magna (Bassi, 1875) Ward, 1917

Site: liver

Host: Bos taurus

Dist.: Alta, Ont

Records: Swales 1933b (Alta); Slocombe 1973a (Ont)

\section{FAMILY HETEROPHYIDAE}

Cryptocotyle lingua (Creplin, 1825) Luhe, 1899

Site: [intestine]

Host: Canis familiaris

Dist.: Nfld, Que

Record: Cameron et al. 1940

\section{FAMILY NANOPHYETIDAE}

Nanophyetus salmincola Chapin, 1927

Site: [intestine]

Host: Canis familiaris

Dist.: BC

Record: Booth et al. 1984

Remarks: Infection was diagnosed by finding $N$. salmincola eggs in the feces.

\section{FAMILY OPISTHORCHIIDAE}

Metorchis conjunctus (Cobbold, 1860) Looss, 1899

Site: bile ducts, pancreatic duct

Hosts: Canis familiaris $(1,2,4,5,6,7,8)$

Felis catus $(3,5,8)$

Dist.: Alta, Man, NWT, Ont, Que, Sask

Records: 1. Cameron et al. 1940 (Man, Ont,

Que, Sask); 2. Mongeau 1961 (Man); 3.

Axelson 1962 (Ont); 4. McLeod 1967 (Man); 5.

Mills and Hirth 1968 (Ont); 6. Unruh et al. 1973

(Alta, NWT, Sask); 7. Anvik et al. 1974 (Sask);

8. Watson 1981 (Que)

Metorchis spp.

Site: [bile ducts]

Host: Canis familiaris

Dist.: Sask

Record: Allen and Mills 1971

Parametorchis complexus (Stiles and Hassall, 1894) Skrjabin, 1913

Syn.: Parametorchis manitobensis (Allen and Wardle, 1934) Kennedy, 1983

Site: bile ducts, gallbladder

Hosts: Canis familiaris (1) Felis catus (2)

Dist.: Alta, Man

Records: 1. Allen and Wardle 1934 (Man); 2.

Kennedy 1983 (Alta)

\section{FAMILY PARAMPHISTOMIDAE}

Calicophoron microbothrioides (Price and McIntosh, 1944) Eduardo, 1983

Syn.: Paramphistomum microbothrioides Price and McIntosh, 1944

Site: rumen

Host: Bos taurus

Dist.: Que

Record: Bouvry and Rau 1984 
Paramphistomum liorchis Fischoeder, 1901

Site: rumen

Host: Bos taurus

Dist.: Que

Record: Bouvry and Rau 1984

Paramphistomum spp.

Site: [intestine]

Host: Bos taurus

Dist.: Alta, Ont

Record: Swales 1933b

\section{FAMILY PLAGIORCHIIDAE}

Plagiorchis massino Petrov and Tikhonov, 1927

Site: [intestine]

Host: Felis catus

Dist.: Nfld

Record: Smith and Threlfall 1973

\section{FAMILY TROGLOTREMATIDAE}

Paragonimus kellicotti Ward, 1908

Site: lungs

Hosts: Canis familiaris $(1,2,3)$

Dist.: Ont

Felis catus (4)

Records: 1. Nielsen 1955; 2. Comfort and

Axelson 1962; 3. Gillick 1972; 4. Rendano 1974

Remarks: Nielsen (1955) presumed his

specimen was $P$. kellicotti.

Paragonimus spp.

Site: lungs

Hosts: Canis familiaris

Dist.: Ont Sus scrofa

Record: Slocombe 1973a

Unidentified Digenea gen. spp.

Site: [intestine]

Host: Canis familiaris

Dist.: Man, NWT, Que

Records: Cameron et al. 1940 (Man, NWT, Que); Seah et al. 1975 (Que); Ghadirian et al. 1976 (Que)

\section{CLASS CESTOIDEA}

\section{SUBCLASS EUCESTODA}

\section{ORDER CYCLOPHYLLIDEA}

\section{FAMILY ANOPLOCEPHALIDAE}

Anoplocephala magna (Abildgaard, 1789)

Site: [intestine]

$$
\text { Spengel, } 1905
$$

Host: Equus caballus

Dist.: Ont, Que

Records: Swales 1933b (Que); Slocombe 1973a

(Ont)
Anoplocephala perfoliata (Goeze, 1782)

Site: [intestine]

Blanchard, 1848

Host: Equus caballus

Dist.: Nfld, Ont

Records: Swales 1933b (Ont); Slocombe 1973a

(Ont); Smith and Threlfall 1973 (Nfld);

Slocombe 1979 (Ont); Slocombe and Cote 1984

(Ont); Slocombe et al. 1987 (Ont)

Anoplocephala spp.

Site: [intestine]

Host: Equus caballus

Dist.: Ont

Records: Swales 1933a; Slocombe 1973a

Moniezia benedeni (Moniez, 1879) Blanchard, 1891

Site: intestine

Host: Bos taurus

Dist.: NB, Que

Records: Swales 1933b (Que); Smith 1970 (NB)

Moniezia expansa (Rudolphi, 1810) Blanchard, 1891

Site: intestine

Hosts: Bos taurus (2)

Ovis aries $(1,2,3,4,5,6,7,8,9,10,11)$

Dist.: Canada, Nfld, NS, Ont, Que

Records: 1. Swales 1933a (unspecified), 2.

1933b (Ont, Que); 3. Griffiths 1937 (Que); 4.

Fallis 1938 (Ont); 5. Swales 1939 (Que), 6.

1940a (Canada), 7. 1940b (Que); 8. Swales and

Choquette 1940 (Que); 9. Swales et al. 1942

(Ont); 10. Smith and Archibald 1965 (NS); 11.

Smith and Threlfall 1973 (Nfld)

Moniezia spp.

Site: [intestine]

Hosts: Bos taurus $(1,2,3)$

Ovis aries $(4,5)$

Dist.: Nfld, Ont, Que, Sask

Records: 1. McGregor and Kingscote 1957

(Ont); 2. Frechette and Gibbs 1971 (Que); 3.

Slocombe 1973a (Ont); 4. Smith and Threlfall

1973 (Nfld); 5. Moteane et al. 1979 (Sask)

Paranoplocephala mamillana (Mehlis, 1831)

Baer, 1927

Syn.: Anoplocephala mamillana (Mehlis, 1831)

Blanchard, 1891

Site: [intestine]

Host: Equus caballus

Dist.: BC, Que, Sask

Record: Swales 1933b

Thysanosoma actinioides Diesing, 1835

Site: [intestine]

Host: Ovis aries

Dist.: Alta, BC, Canada, Sask

Records: Swales 1933a (BC), 1933b (Alta,

Sask), 1940a (Canada)

Thysanosoma spp.

Site: bile duct

Host: Ovis aries

Dist.: Ont, Sask

Records: Slocombe 1973a (Ont); Moteane et al. 1979 (Sask) 


\section{FAMILY DILEPIDIDAE}

Dipylidium caninum (Linnaeus, 1758) Railliet, 1892

Site: [intestine]

Hosts: Canis familiaris $(1,2,3,5,6,7)$

Felis catus $(1,4)$

Dist.: NB, Nfld, NWT, Ont, Que

Records: 1. Swales 1933b (Ont, Que); 2.

Cameron et al. 1940 (NWT, Ont); 3. Freeman

1962 (Ont); 4. Threlfall 1969 (Nfld); 5. Smith

and Threlfall 1973 (Nfld); 6. Unruh et al. 1973

(NWT); 7. Malloy and Embil 1978 (NB)

Dipylidium spp.

Site: [intestine]

Hosts: Canis familiaris $(1,2)$

Felis catus (2)

Dist.: Ont, Sask

Records: 1. Allen and Mills 1971 (Sask); 2.

Slocombe 1973a (Ont)

\section{FAMILY MESOCESTOIDIDAE}

Mesocestoides spp.

Site: intestine

Host: Canis familiaris

Dist.: Ont

Record: Speckmann and Webster 1975

\section{FAMILY TAENIIDAE}

Echinococcus granulosus (Batsch, 1786) Rudolphi, 1801

Site: intestine

Host: Canis familiaris

Dist.: Ont, Que

Records: Choquette 1956 (Que); Slocombe 1973a (Ont)

Echinococcus granulosus (Batsch, 1786) Rudolphi, 1801 (hydatid cyst)

Site: liver

Host: Sus scrofa

Dist.: Ont

Record: Pallister and Plummer 1941 (Ont)

Echinococcus multilocularis Leuckart, 1863

Site: [intestine]

Host: Felis catus

Dist.: Sask

Record: Wobeser 1971

Echinococcus spp. (hydatid)

Site: liver

Host: Sus scrofa

Dist.: Ont

Record: Osler and Clement 1883

Multiceps spp.

Site: unspecified

Host: Sus scrofa

Dist.: Ont

Record: Slocombe 1973a
Taenia crassiceps (Zeder, 1800) Rudolphi, 1810

Site: [intestine]

Hosts: Canis familiaris (2)

Dist.: Ont

Canis familiaris $\mathrm{x}$ Canis latrans (1)

Records: 1. Freeman et al. 1961; 2. Freeman 1962.

Taenia elliptica

Site: intestine

Host: Canis familiaris

Dist.: Que

Record: Osler 1877

Taenia hydatigena (Pallas, 1766)

Syn: Cysticercus tenuicollis Pallas, 1766

Site: mesenteries, peritoneum, intestine

Hosts: Bos taurus (1)

Canis familiaris $(1,3,4)$

Ovis aries $(1,2,3,5,6)$

Sus scrofa (3)

Dist.: Alta, Canada, Nfld, Ont, Que, Sask

Records: 1. Swales 1933b (Alta, Ont, Que); 2.

Swales 1940a (Canada); 3. Sweatman and

Plummer 1957 (Que); 4. Threlfall 1969 (Nfld);

5. Slocombe 1973a (Ont); 6. Moteane et al. 1979 (Sask)

Taenia ovis (Cobbold, 1869), Ransom, 1913

Syn: Cysticercus ovis

Site: unspecified

Host: Ovis aries

Dist.: Canada, Man, NS

Record: Swales 1933b (Man), 1940a (Canada);

Soehl 1984a (NS), 1984b (NS)

Taenia pisiformis (Bloch, 1780) Gmelin, 1790

Site: [intestine]

Hosts: Canis familiaris $(1,3,4,5)$

C. familiaris $\mathrm{x} C$. latrans (2)

Dist.: Alta, Nfld, NWT, Ont, Que

Records: 1. Swales 1933b (Que); 2. Freeman et al. 1961 (Ont); 3. Threlfall 1969 (Nfld); 4. Smith and Threlfall 1973 (Nfld); 5. Unruh et al. 1973 (Alta, NWT)

Taenia serialis (Gervais, 1847) Baillet, 1863

Site: [intestine]

Host: Canis familiaris

Dist.: Alta, NWT

Record: Unruh et al. 1973

Taenia solium Linnaeus, 1758

Syn: Cysticercus cellulosae Linnaeus, 1758

Site: [liver]

Host: Sus scrofa

Dist.: Man, Ont, Sask

Records: Osler and Clement 1883 (Ont); Swales 1933b (Man, Sask)

Taenia taeniaeformis (Batsch, 1786) Wolffgel, 1863

Site: [intestine]

Host: Felis catus

Dist.: Nfld, Que

Records: Swales 1933b (Que); Smith and

Threlfall 1973 (Nfld) 
Taenia spp.

Site: [intestine]

Hosts: Canis familiaris $(1,2,3)$

Felis catus $(2,4)$

Dist.: BC, Man, NWT, Ont, Que, Sask

Records: 1. Cameron 1940 (BC, Man, NWT,

Ont, Que); 2. Slocombe 1973a (Ont); 3. Anvik

et al. 1974 (Sask); 4. Rendano 1974 (Ont)

Taeniarhyncus saginatus (Goeze, 1782)

Weinland, 1858

Syn: Cysticercus bovis Cobbold, 1866

Site: unspecified

Host: Bos taurus

Dist.: Ont, Sask

Records: Swales 1933b (Ont, Sask); Slocombe 1973a (Ont)

Taenia/ Echinococcus spp. (egg)

Site: [intestine]

Host: Canis familiaris

Dist.: Alta, NWT, Sask

Records: Allen and Mills 1971 (Sask); Unruh et al. 1973 (Alta, NWT, Sask)

\section{ORDER PSEUDOPHYLLIDEA}

\section{FAMILY DIPHYLLOBOTHRIIDAE}

Diphyllobothrium dendriticum Nitsch, 1824

Site: [intestine]

Host: Felis catus

Dist.: Nfld

Records: Threlfall 1969; Smith and Threlfall

1973

Diphyllobothrium latum Luhe, 1910

Site: [intestine]

Host: Canis familiaris

Dist.: Alta, Nfld, NWT, Que, Sask

Records: Saunders 1949 (Alta); Threlfall 1969

(Nfld); Smith and Threlfall 1973 (Nfld); Unruh

et al. 1973 (NWT); Anvik et al. 1974 (Sask);

Seah et al. 1975 (Que)

Diphyllobothrium spp.

Site: [intestine]

Hosts: Canis familiaris $(1,2,3,4,6)$

Felis catus (5)

Dist.: Alta, Man, Nfld, NWT, Ont, Que, Sask

Records: 1. Wardle and McColl 1937 (Ont); 2.

Cameron et al. 1940 (Alta, Man, Nfld, NWT,

Ont, Que, Sask); 3. Threlfall 1969 (Nfld); 4.

Allen and Mills 1971 (Sask); 5. Slocombe 1973a

(Ont); 6. Unruh et al. 1973 (Alta, NWT, Sask)

Unidentified Cestoidea gen. spp.

Site: Intestine

Host: Canis familiaris

Dist.: Man, Que

Records: Mongeau 1961 (Man); Seah et al.

1975 (Que)
PHYLUM NEMATODA

SUBCLASS ADENOPHOREA

ORDER ENOPLIDA

SUPERFAMILY DIOCTOPHYMATOIDEA

FAMILY DIOCTOPHYMATIDAE

Dioctophyma renale (Goeze, 1782) Collet-Meygret, 1802

Site: kidney

Host: Canis familiaris

Dist.: Man, NWT, Ont, Que

Records: Swales 1933b (Ont, Que); Cameron et al. 1940 (Man); McLeod 1967 (Man); Unruh et al. 1973 (NWT)

\section{SUPERFAMILY TRICHINELLOIDEA}

\section{FAMILY TRICHINELLIDAE}

Trichinella spiralis (Owen, 1835) Railliet, 1894 (cyst/larva)

Site: diaphragm

Host: Sus scrofa

Dist.: BC, Man, NB, NS, Ont, PEI, Que

Records: Osler and Clement 1883 (Ont);

Cameron 1938 (Man, Ont, PEI, Que), 1939

(Man, Ont, PEI, Que), 1940a (Que); Moynihan and Musfeldt 1949 (BC); Frank 1952 (NB, NS, PEI); Tailyour and Steele 1960 (BC); Smith 1975a (NB); Faubert et al. 1985 (Que)

Remarks: Faubert et al. (1985) used the ELISA technique to detect $T$. spiralis.

Trichinella spiralis spiralis (Owen, 1835)

Site: unspecified

Host: Sus scrofa

Dist.: NS

Record: Smith and Kay 1987 (NS)

Trichinella spp.

Site: tail musculature

Host: Sus scrofa

Dist.: NB

Record: Smith 1975b

\section{FAMILY TRICHURIDAE}

Capillaria aerophila Creplin, 1839

Site: [lungs]

Hosts: Canis familiaris (2)

Dist.: Que Felis catus (1)

Record: 1. Rendano 1974 (Ont); 2. Seah et al. 1975 (Que)

Capillaria hepatica Bancroft, 1896

Site: liver

Hosts: Felis catus (1)

Dist.: Alta, NB

Equus caballus (2)

Records: 1. Malloy and Embil 1978 (NB); 2.

Nation and Dies 1978 (Alta) 
Capillaria longipes Ransom, 1911

Site: [intestine]

Host: Ovis aries

Dist.: Canada, Que

Records: Swales 1939 (Que), 1940a (Canada);

Ayalew and Gibbs 1973 (Que)

Capillaria spp.

Site: unspecified

Hosts: Bos taurus (3,5)

Canis familiaris (7)

Felis catus (7)

Ovis aries $(1,2,4,6)$

Dist.: NS, Ont, Que

Records: 1. Griffiths 1937 (Que); 2. Fallis 1938

(Ont); 3. McGregor and Kingscote 1957 (Ont);

4. Smith and Archibald 1965 (NS); 5. Frechette and Gibbs 1971 (Que); 6. Ayalew et al. 1973a

(Que); 7. Slocombe 1973a (Ont)

Trichuris discolor Linstou, 1906

Site: [intestine]

Host: Bos taurus

Dist.: NB, Ont, Que

Records: Smith and Stevenson 1970 (NB);

Frechette et al. 1973 (Que); Slocombe 1974b

(Ont)

Trichuris ovis (Abildgaard, 1795) Smith, 1908

Site: [intestine]

Hosts: Bos taurus $(1,7)$

Ovis aries $(1,2,3,4,5,6,7,8,9,10)$

Dist.: Canada, NB, Nfld, NS, Ont, Que

Records: 1. Swales 1933b (NS, Ont, Que); 2.

Fallis 1938 (Ont); 3. Swales 1940a (Canada), 4.

1940b (Que); 5. Swales and Choquette 1940

(Que); 6. Smith and Archibald 1965 (NS), 7.

1968 (NB); 8. Ayalew and Gibbs 1973 (Que); 9.

Ayalew et al. 1973a (Que); 10. Smith and

Threlfall 1973 (Nfld)

Trichuris suis (Shrank, 1788)

Site: [intestine]

Host: Sus scrofa

Dist.: NB, Ont, Que

Records: Swales 1933b (Ont, Que); Smith 1972

(NB); Martin et al. 1974 (Que)

Trichuris vulpis (Frohlich, 1789)

Site: [intestine]

Host: Canis familiaris

Dist.: Nfld, Ont, Que

Records: Osler 1877 (Que); Swales 1933b (Ont,

Que); Cameron et al. 1940 (Ont); Choquette

and Gelinas 1950 (Que); Smith and Threlfall

1973 (Nfld); Seah et al. 1975 (Que)

Trichuris spp.

Site: [intestine]

Hosts: Bos taurus $(3,4,5,6)$

Canis familiaris $(2,4)$

Ovis aries $(1,4)$

Sus scrofa (2)

Dist.: Alta, NWT, Ont, Sask
Records: 1. Swales et al. 1942 (Ont); 2.

Slocombe 1973a (Ont), 3. 1973b (Ont); 4.

Unruh et al. 1973 (Alta, NWT, Sask); 5.

Moteane et al. 1979 (Sask); 6. Reid and Madill

1987 (Ont)

Trichuris spp. (egg)

Site: [intestine]

Hosts: Bos taurus $(1,2)$

Canis familiaris $(3,4,5)$

Dist.: NB, Ont, Que, Sask

Records: 1. McGregor and Kingscote 1957

(Ont); 2. Smith 1970 (NB); 3. Allen and Mills

1971 (Sask); 4. Ghadirian et al. 1976 (Que); 5.

Malloy and Embil 1978 (NB)

Unidentified Trichuridae

Site: [intestine]

Host: Bos taurus

Dist.: Ont

Record: Campbell et al. 1960

SUBCLASS SECERNENTEA

ORDER ASCARIDIDA

\section{SUPERFAMILY ASCARIDOIDEA}

\section{FAMILY ASCARIDIDAE}

Ascaris lumbricoides Linnaeus, 1758 (larva)

Site: lungs

Hosts: Bos taurus (3)

Sus scrofa $(1,2)$

Dist.: All Provinces, Ont

Records: 1. Swales 1933a (Ont), 2. 1933b (All

Provinces); 3. Allen 1962 (Ont)

Ascaris suum Goeze, 1782

Site: [intestine]

Hosts: Bos taurus (1)

Ovis aries (6)

Sus scrofa $(2,3,4,5)$

Dist.: Ont, NB, Nfld, Que, Sask

Records: 1. McCraw and Lautenslager 1971

(Ont); 2. Smith 1972 (NB); 3. Smith and

Threlfall 1973 (Nfld); 4. Martin et al. 1974

(Que); 5. Polley and Mostert 1980 (Sask); 6.

Sauvageau and Frechette 1980 (Que)

Ascaris spp.

Site: [intestine]

Hosts: Canis familiaris (4)

Ovis aries $(1,2)$

Sus scrofa (3)

Dist.: Canada, Ont, Que

Records: 1. Fallis 1938 (Ont); 2. Swales 1940a

(Canada); 3. Slocombe 1973a (Ont); 4. Seah et al. 1975 (Que)

Parascaris equorum (Goeze, 1782) York and

Site: [intestine]
Maplestone, 1926 
Host: Equus caballus

Dist.: NB, Nfld, Ont, Que

Records: Swales 1933b (Ont, Que); Smith and

Threlfall 1973 (Nfld); Smith 1976a (NB);

Slocombe and Cote 1984 (Ont); Slocombe et al. 1987 (Ont)

Parascaris spp.

Site: [intestine]

Host: Equus caballus

Dist.: Ont

Records: Slocombe 1973a; Slocombe and McCraw 1973

Toxascaris leonina (Linstow, 1902) Leiper, 1907

Syn.: Toxascaris limbata Railliet and Henry,

1911

Site: intestine

Hosts: Canis familiaris $(1,2,3,4,5,6,7,8,9,10)$

Felis catus $(5,6,10)$

Dist.: Alta, Man, NB, Nfld, NWT, Ont, Que,

Sask

Records: 1. Osler 1877 (Que); 2. Swales 1933b

(Que); 3. Cameron et al. 1940 (Alta, Man, NB,

Nfld, Ont, Que, Sask); 4. Choquette and

Gelinas 1950 (Que); 5. Threlfall 1969 (Nfld); 6.

Smith and Threlfall 1973 (Nfld); 7. Unruh et al.

1973 (Alta, NWT, Sask); 8. Anvik et al. 1974

(Sask); 9. Ghadirian et al. 1976 (Que); 10.

Malloy and Embil 1978 (NB)

Toxascaris spp.

Site: [intestine]

Hosts: Canis familiaris $(1,2)$ Felis catus (2)

Dist.: Ont, Sask

Records: 1. Allen and Mills 1971 (Sask); 2.

Slocombe 1973a (Ont)

Toxocara canis (Werner, 1782) Stiles, 1905

Site: intestine

Host: Canis familiaris

Dist.: Alta, Man, NB, Nfld, NS, NWT, Ont,

Que, Sask

Records: Cameron et al. 1940 (Alta, Man, Nfld,

NWT, Ont, Que, Sask); Choquette and Gelinas

1950 (Que); Webster 1958 (Que); Threlfall 1969

(Nfld); Malloy and Embil 1973 (NS); Smith and Threlfall 1973 (Nfld); Unruh et al. 1973

(Alta, NWT, Sask); Anvik et al. 1974 (Sask);

Seah et al. 1975 (Que); Ghadirian et al. 1976

(Que); Malloy and Embil 1978 (NB)

Toxocara cati (Schrank, 1788) Brumpt, 1927

Syn.: Belascaris mystax Zeder, 1800

Syn.: Toxocara mystax (Zeder, 1800)

Site: [intestine]

Host: Felis catus

Dist.: NB, Nfld, NS, Ont, Que

Records: Swales 1933b (Que); Threlfall 1969

(Nfld); Malloy and Embil 1973 (NS); Smith

and Threlfall 1973 (Nfld); Rendano 1974 (Ont);

Malloy and Embil 1978 (NB)
Toxocara spp.

Site: [intestine]

Hosts: Canis familiaris $(1,2)$

Felis catus (2)

Dist.: Ont, Sask

Records: 1. Allen and Mills 1971 (Sask); 2.

Slocombe 1973a (Ont)

\section{SUPERFAMILY COSMOCERCOIDEA}

\section{FAMILY ATRACTIDAE}

Probstmayria vivipara (Probstmayr, 1865) Ransom 1907

Site: caecum, colon

Host: Equus caballus

Dist.: NB, Que

Records: Swales 1933a (unspecified), 1933b

(Que); Smith 1976a (NB), 1976b (NB), 1979b

(NB)

\section{ORDER OXYURIDA}

\section{SUPERFAMILY OXYUROIDEA}

\section{FAMILY OXYURIDAE}

Oxyuris equi (Schrank, 1788) Rudolphi, 1803

Site: [intestine]

Host: Equus caballus

Dist.: NB, Nfld, Ont, Que

Records: Swales 1933b (Ont, Que); Threlfall

1969 (Nfld); Smith 1976a (NB)

Oxyuris spp.

Site: [intestine]

Host: Equus caballus

Dist.: Ont

Records: Slocombe 1973a; Slocombe and

McCraw 1973

Skrjabinema spp.

Site: [intestine]

Host: Ovis aries

Dist.: Sask

Record: Moteane et al. 1979

\section{ORDER RHABDITIDA}

\section{SUPERFAMILY RHABDITOIDEA}

\section{FAMILY RHABDITIDAE}

Pelodera strongyloides (Schneider, 1860) Schneider, 1866

Syn.: Rhabditis strongyloides (Schneider, 1860)

Oerley, 1880

Site: unspecified

Host: Canis familiaris

Dist.: Ont

Record: Lord and McGregor 1960 
FAMILY STRONGYLOIDIDAE

Strongyloides papillosus (Wedl, 1856) Ransom, 1911

Site: intestine

Hosts: Bos taurus (7)

Ovis aries $(1,2,3,4,5,6,8,9)$

Dist.: Canada, NS, Ont, Que

Records: 1. Griffiths 1937 (Que); 2. Fallis 1938

(Ont); 3. Swales 1940a (Canada), 4. 1940b

(Que); 5. Basir 1950 (Que); 6. Smith and

Archibald 1965 (NS); 7. Frechette and Gibbs

1971 (Que); 8. Ayalew and Gibbs 1973 (Que); 9.

Ayalew et al. 1973a (Que)

Strongyloides ransomi Schwartz and Alicata, 1930

Site: intestine

Host: Sus scrofa

Dist.: NB

Record: Smith 1972

Strongyloides stercoralis (Bavay, 1876) Stiles and Hassall, 1902

Site: unspecified

Host: Canis familiaris

Dist.: Que

Records: Choquette and Gelinas 1950; Seah et al. 1975

Strongyloides spp.

Site: unspecified

Hosts: Bos taurus $(3,4)$

Canis familiaris (5)

Equus caballus $(5,6)$

Felis catus (5)

Ovis aries $(1,2,7)$

Sus scrofa (5)

Dist.: Ont, Que, Sask

Records: 1. Swales 1939 (Que); 2. Swales et al.

1942 (Ont); 3. McGregor and Kingscote 1957

(Ont); 4. Slocombe 1973a (Ont), 5. 1973b (Ont);

6. Slocombe and McCraw 1973 (Ont); 7.

Moteane et al. 1979 (Sask)

Unidentified Rhabditoidea

Site: unspecified

Hosts: Bos taurus (1)

Canis familiaris (2)

Dist.: Ont

Felis catus (2)

Records: 1. McGregor and Kingscote 1957; 2.

Slocombe 1973a

\section{ORDER SPIRURIDA}

\section{SUPERFAMILY DRACUNCULOIDEA}

\section{FAMILY DRACUNCULIDAE}

Dracunculus insignis (Leidy, 1858) Chandler, 1942

Site: unspecified

Host: Canis familiaris

Dist.: Que

Record: Deroth and Frechette 1975

\section{SUPERFAMILY FILARIOIDEA}

FAMILY FILARIIDAE

Parafilaria bovicola Tubangui, 1934

Site: subcutaneous tissue

Host: Bos taurus

Dist.: Alta, Que

Records: Niilo 1968 (Alta); Webster and

Wilkins 1970 (Que)

Remarks: Both records were from imported cattle. Niilo's (1968) record was a presumptive identification.

Stephanofilaria stilesi Chitwood, 1934

Site: subcutaneous

Host: Bos taurus

Dist.: Alta

Record: Maddy 1955; Dies and Pritchard 1985

Remarks: The infection was diagnosed in the

United States in cattle imported from Alberta

(Maddy 1955).

\section{FAMILY ONCHOCERCIDAE}

\section{Dipetalonema spp.}

Site: unspecified

Host: Canis familiaris

Dist.: Ont

Record: Slocombe 1973a

Dirofilaria immitis (Leidy, 1856) Railliet and Henry, 1911

Site: pulmonary artery, right ventricle, blood

Hosts: Canis familiaris $(1,3,4,5,6)$ Felis catus (2)

Dist.: Alta, Ont, Sask

Records: 1. Fletch 1969 (Ont); 2. Bernard 1970

(Ont), 3. 1971 (Ont); 4. Bourns et al. 1975 (Ont);

5. Frimeth and Arai 1983 (Alta); 6. Stogdale 1984 (Sask)

Remarks: The dog in Bernard's (1971) report was originally diagnosed in the United States. The dog in Stogdale's (1984) report was born and raised until four months of age in Ontario. From there it had been brought directly to Saskatchewan and was diagnosed positive at fourteen years of age. Diagnosis was based on an indirect immunofluorescent antibody test.

\section{Dirofilaria spp.}

Site: unspecified

Host: Canis familiaris

Dist.: Ont

Record: Slocombe 1973a

Onchocerca cervicalis Railliet and Henry, 1910

Site: unspecified

Host: Equus caballus

Dist.: BC, Que

Records: Marcoux et al. 1977 (Que); Lees et al. 1983 (BC) 
Onchocerca gutturosa Neumann, 1910

Site: ligamentum nuchae

Host: Bos taurus

Dist.: Ont, Que

Record: Webster and Dukes 1979

Onchocerca lienalis Stiles, 1892

Site: ligamentum gastrolienale

Host: Bos taurus

Dist.: Ont, Que

Record: Webster and Dukes 1979

Onchocerca spp.

Site: subcutaneous nodules in area of knee joint

Hosts: Bos taurus $(1,2)$

$$
\text { Equus caballus (3) }
$$

Dist.: Alta, BC, Man, Sask

Records: 1. Webster et al. 1977 (Man, Sask); 2.

Dukes et al. 1981 (Man, Sask); 3. Polley 1984

(Alta, BC, Man, Sask)

Remarks: Polley 1984 did not record the

infection rate by province.

Setaria equina (Abildgaard, 1789) Railliet and Henry, 1911

Site: unspecified

Host: Equus caballus

Dist.: Ont, Que

Record: Swales 1933b

Setaria labiatopapillosa (Perroncito, 1882) Railliet and Henry, 1911

Site: unspecified

Host: Bos taurus

Dist.: Alta, Man, Ont, Que

Record: Swales 1933b

Setaria spp.

Site: peritoneum

Host: Equus caballus

Dist.: Ont

Record: Slocombe 1973a

\section{SUPERFAMILY HABRONEMATOIDEA}

\section{FAMILY HABRONEMATIDAE}

Habronema majus (Creplin, 1849) Railliet, 1923

Syn.: Habronema microstoma (Schnider, 1866)

Site: unspecified

Host: Equus caballus

Dist.: Ont

Record: Swales 1933b

Habronema muscae (Carter, 1861) Diesing, 1861

Site: unspecified

Host: Equus caballus

Dist.: Ont

Record: Swales 1933b

Habronema spp.

Site: unspecified

Host: Equus caballus

Dist.: Ont

Record: Slocombe 1973a
SUPERFAMILY PHYSALOPTEROIDEA

FAMILY PHYSALOPTERIDAE

Physaloptera rara Hall and Wigdor, 1918

Site: [intestine]

Host: Canis familiaris

Dist.: Que

Record: Seah et al. 1975

SUPERFAMILY SPIRUROIDEA

FAMILY GONGYLONEMATIDAE

Gongylonema pulchrum Molin, 1857

Site: unspecified

Host: Ovis aries

Dist.: Canada, Ont

Records: Swales 1933b (Ont), 1940a (Canada)

\section{FAMILY SPIROCERCIDAE}

Ascarops spp.

Site: stomach

Host: Sus scrofa

Dist.: Ont

Record: Stockdale and Lautenslager 1973

Physocephalus spp.

Site: stomach

Host: Sus scrofa

Dist.: Ont

Record: Stockdale and Lautenslager 1973

Spirocerca spp.

Site: unspecified

Host: Canis familiaris

Dist.: Ont

Record: Slocombe 1973a

SUPERFAMILY THELAZIOIDEA

FAMILY THELAZIIDAE

Thelazia gulosa Railliet and Henry, 1910

Site: unspecified

Host: Bos taurus

Dist.: Ont

Record: Moolenbeek and Surgeoner 1980

Thelazia lacrymalis (Gurlt, 1831) Railliet and Henry, 1910

Site: conjunctiva, nasolacrimal ducts, orbit of eye

Hosts: Bos taurus (3)

Equus caballus $(1,2)$

Dist.: Ont, Que

Records: 1. Barker 1970 (Ont); 2. Frechette et al. 1976 (Que); 3. Moolenbeek and Surgeoner 1980

Thelazia skrjabini Erschow, 1928

Site: lacrimal ducts, under the nictitating membrane

Host: Bos taurus

Dist.: Alta

Record: Kennedy and Moraiko 1987 
Thelazia spp.

Site: unspecified

Host: Bos taurus (2) Equus caballus (1)

Dist.: Ont, Que

Record: 1. Slocombe 1973a (Ont) 2. Frechette et al. 1976 (Que)

\section{ORDER STRONGYLIDA}

\section{SUPERFAMILY ANCYLOSTOMATOIDEA}

\section{FAMILY ANCYLOSTOMATIDAE}

Ancylostomum caninum Ercolani, 1854

Site: [intestine]

Host: Canis familiaris

Dist.: Alta, Ont, Que

Records: Osler 1877 (Que); Swales 1933b (Ont); Cameron et al. 1940 (Ont, Que); Saunders 1949 (Alta)

Ancylostomum spp.

Site: [intestine]

Hosts: Canis familiaris $(1,2)$

$$
\text { Felis catus (1) }
$$

Dist.: Ont, Sask

Records: 1. Slocombe 1973a (Ont); 2. Anvik et al. 1974 (Sask)

Bunostomum phlebotomum (Railliet, 1900) Lane, 1917

Syn.: Monodontus phlebotomus Railliet, 1900

Site: unspecified

Host: Bos taurus

Dist.: Que

Record: Swales 1933b

Bunostomum trigonocephalum (Rudolphi, 1808)

Railliet, 1902

Syn.: Monodontus trigonocephalus Rudolphi,

1808

Site: unspecified

Hosts: Bos taurus (1)

Ovis aries $(1,2,3,4,5,6,7,8,9,10)$

Dist.: Canada, Nfld, NS, Ont, Que

Records: 1. Swales 1933b (Ont, Que), 2.

Griffiths 1937 (Que); 3. Swales 1940a (Canada);

4. Fallis 1938 (Ont); 5 . Swales and Choquette 1940 (Que); 6. Swales et al. 1942 (Ont); 7. Smith and Archibald 1965 (NS); 8. Ayalew and Gibbs 1973 (Que); 9. Ayalew et al. 1973a (Que); 10.

Smith and Threlfall 1973 (Nfld)

\section{Bunostomum spp.}

Syn.: Monodontus spp.

Site: unspecified

Hosts: Bos taurus $(2,3)$

Ovis aries $(1,4)$

Dist.: Ont, Que, Sask

Records: 1. Swales 1939 (Que); 2. Frechette and Gibbs 1971 (Que); 3. Slocombe 1973b (Ont); 4.

Moteane et al. 1979 (Sask)
Uncinaria stenocephala (Railliet, 1884) Railliet, 1885

Site: [intestine]

Host: Canis familiaris

Dist.: Alta, BC, Man, Nfld, NWT, Ont, Que

Records: Cameron et al. 1940 (Alta, BC, Man, Nfld, NWT, Ont, Que); Threlfall 1969 (Nfld); Smith and Threlfall 1973 (Nfld); Unruh et al. 1973 (Alta, NWT)

Uncinaria spp.

Site: [intestine]

Host: Canis familiaris

Dist.: Ont, Sask

Records: Slocombe 1973a (Ont); Anvik et al. 1974 (Sask)

Unidentified Ancylostomatidae

Site: unspecified

Host: Canis familiaris

Dist.: Alta, NB, NWT, Que, Sask

Records: Allen and Mills 1971 (Sask); Unruh et al. 1973 (Alta, NWT); Seah et al. 1975 (Que);

Ghadirian et al. 1976 (Que); Malloy and Embil 1978 (NB); Olson 1985 (Sask)

\section{SUPERFAMILY METASTRONGYLOIDEA}

\section{FAMILY ANGIOSTRONGYLIDAE}

Aelurostrongylus spp.

Site: unspecified

Host: Felis catus

Dist.: Ont

Record: Slocombe 1973a

\section{FAMILY CRENOSOMATIDAE}

Crenosoma vulpis (Dujardin, 1844) Railliet, 1915

Site: [lungs]

Host: Canis familiaris

Dist.: Nfld

Record: Smith and Threlfall 1973

\section{FAMILY FILAROIDIDAE}

Filaroides milksi Whitlock, 1956

Site: terminal and respiratory bronchioles

Host: Canis familiaris

Dist.: BC

Record: Greenway and Stockdale 1970

Remarks: This was a tentative identification

(Greenway and Stockdale 1970)

Filaroides spp.

Site: unspecified

Host: Canis familiaris

Dist.: Ont

Record: Slocombe 1973a

Oslerus osleri (Cobbold, 1879) Anderson, Chabaud, and Willmott, 1978

Site: bronchi

Host: Canis familiaris

Dist.: Que

Record: Osler 1877 


\section{FAMILY METASTRONGYLIDAE}

Metastrongylus apri (Gmelin, 1790) Vostokov, 1905

Site: bronchii

Host: Sus scrofa

Dist.: NB

Record: Smith 1972

Metastrongylus pudendotectus Vostokov, 1905

Site: unspecified

Host: Sus scrofa

Dist.: Ont

Record: Swales 1933b

Metastrongylus salmi Gedoelst, 1923

Syn.: Metastrongylus elongatus Salm, 1918

Site: unspecified

Host: Sus scrofa

Dist.: NS, Ont, PEI, Que

Record: Swales 1933b

Metastrongylus spp.

Site: unspecified

Host: Sus scrofa

Dist.: Ont

Record: Slocombe 1973a

\section{FAMILY PROTOSTRONGYLIDAE}

Muellerius capillaris (Mueller, 1889) Cameron, 1927

Site: lungs

Hosts: Capra hircus $(3,4)$

Ovis aries $(1,2)$

Dist.: Canada, Ont

Records: 1. Fallis 1938 (Ont); 2. Swales 1940a

(Canada); 3. McCraw et al. 1981 (Ont); 4.

McCraw and Menzies 1986 (Ont)

Muellerius spp.

Site: unspecified

Hosts: Capra hircus (2)

Dist.: Ont

Ovis aries (1)

Record: 1. Slocombe 1973a; 2. Nimmo 1979

Parelaphostrongylus tenuis (Dougherty, 1945)

Pryadko and Boev, 1971

Syn.: Pneumostrongylus tenuis Dougherty,

1945

Site: central nervous system

Host: Ovis aries

Dist.: Ont

Record: Anderson and Strelive 1966.

Protostrongylus spp.

Site: unspecified

Host: Ovis aries

Dist.: Ont

Record: Slocombe 1973a

\section{SUPERFAMILY STRONGYLOIDEA}

\section{FAMILY CHABERTIIDAE}

Chabertia ovina (Fabricius, 1794) Railliet and Henry, 1909

Site: [intestine]

\section{Host: Ovis aries}

Dist.: Canada, NS, Ont, Que

Records: Swales 1933b (Ont); Griffiths 1937

(Que); Fallis 1938 (Ont); Swales 1939 (Que);

Swales 1940a (Canada), 1940b (Que); Swales

and Choquette 1940 (Que); Smith and

Archibald 1965 (NS); Ayalew and Gibbs 1973

(Que); Ayalew et al. 1973a (Que)

Chabertia spp.

Site: [intestine]

Host: Ovis aries

Dist.: Ont, Sask

Records: Swales et al. 1942 (Ont); Slocombe

1973a (Ont); Moteane et al. 1979 (Sask)

Oesophagostomum columbianum (Curtice, 1890)

Site: [intestine]

Stossich, 1899

Host: Ovis aries

Dist.: Canada, NS, Ont, Que

Records: Swales 1933b(NS, Ont, Que); Fallis

1938 (Ont); Swales 1939 (Que), 1940a

(Canada), 1940b (Que); Swales and Choquette 1940 (Que); Swales et al. 1942 (Ont); Smith and Archibald 1965 (NS); Ayalew and Gibbs 1973

(Que); Ayalew et al. 1973a (Que)

Oesophagostomum dentatum (Rudolphi, 1803)

Site: [intestine]

Molin, 1861

Host: Sus scrofa

Dist.: NB, Ont, Que

Records: Swales 1933b (Ont, Que); Smith 1972

(NB), 1979a (NB)

Oesophagostomum quadrispinulatum (Marcone, 1901) Alicata, 1935

Site: [intestine]

Host: Sus scrofa

Dist.: NB

Records: Smith 1972, 1979a

Oesophagostomum radiatum (Rudolphi, 1803)

Site: [intestine]

Railliet, 1898

Host: Bos taurus

Dist.: NB, Nfld, Ont, Que

Records: Swales 1933b (Ont, Que); Smith and

Perreault 1972 (NB); Smith and Threlfall 1973

(Nfld)

Oesophagostomum venulosum (Rudolphi, 1809)

Railliet and Henry,

Site: [intestine]

1913

Host: Ovis aries

Dist.: Canada, Nfld, NS, Ont, Que

Records: Swales 1933b (Ont, Que), 1940a

(Canada); Smith and Archibald 1965 (NS);

Ayalew and Gibbs 1973 (Que); Smith and

Threlfall 1973 (Nfld)

Oesophagostomum spp.

Site: intestine

Hosts: Bos taurus $(3,4)$ 
Ovis aries $(1,4,5)$

Sus scrofa $(2,4,6,7)$

Dist.: NB, Nfld, Ont, Que

Records: 1. Swales 1939 (Que); 2. Davenport and Stockdale 1967 (Ont); 3. Frechette and Gibbs 1971 (Que); 4. Slocombe 1973b (Ont); 5. Smith and Threlfall 1973 (Nfld); 6. Martin et al. 1974 (Que); 7. Smith 1979a (NB)

\section{FAMILY STRONGYLIDAE}

Cyathostomum catinatum Looss, 1900

Syn.: Cylicostomum catinatum (Looss, 1900)

Gedoelst, 1924

Syn.: Trichonema catinatum (Looss, 1900)

LeRoux, 1924

Site: [intestine]

Host: Equus caballus

Dist.: Alta, Nfld, Sask

Records: Ransom and Hadwen 1918 (Alta,

Sask); Smith and Threlfall 1973 (Nfld)

Cyathostomum coronatum Looss, 1900

Syn.: Cylicostomum coronatum (Looss, 1900)

Gedoelst, 1903

Site: [intestine]

Host: Equus caballus

Dist.: Sask

Record: Ransom and Hadwen 1918

Cyathostomum labiatum (Looss, 1902) McIntosh, 1933

Syn.: Cylicostomum labiatum (Looss, 1902)

Gedoelst, 1903

Site: [intestine]

Host: Equus caballus

Dist.: Alta, Sask

Record: Ransom and Hadwen 1918

Cyathostomum labratum Looss, 1900

Syn.: Cylicostomum labratum (Looss, 1900)

Gedoelst, 1903

Site: [intestine]

Host: Equus caballus

Dist.: Alta, Sask

Record: Ransom and Hadwen 1918

Cylicocyclus elongatus (Looss, 1900) Chaves, 1930

Syn.: Cylicostomum elongatum (Looss, 1900)

Gedoelst, 1903

Site: [intestine]

Host: Equus caballus

Dist.: Nfld, Sask

Records: Ransom and Hadwen 1918 (Sask);

Smith and Threlfall 1973 (Nfld)

Cylicocyclus insigne (Boulenger, 1917) Chaves, 1930

Syn.: Cylicostomum insigne (Boulenger, 1917)

Ransom and Hadwen, 1918

Site: [intestine]

Host: Equus caballus

Dist.: Alta

Record: Ransom and Hadwen 1918
Cylicocyclus nassatus (Looss, 1900) Chaves, 1930

Syn.: Cylicostomum nassatum (Looss, 1900)

Gedoelst, 1903

Site: [intestine]

Host: Equus caballus

Dist.: Alta, Nfld, Sask

Records: Ransom and Hadwen 1918 (Alta,

Sask); Smith and Threlfall 1973 (Nfld)

Cylicocyclus radiatus (Looss, 1900) Chaves, 1930

Syn.: Cylicostomum radiatum (Looss, 1900)

Gedoelst, 1903

Site: [intestine]

Host: Equus caballus

Dist.: Alta, Sask

Record: Ransom and Hadwen 1918

Cylicodontophorus bicornatus (Looss, 1900) Cram, 1924

Syn.: Cylicostomum bicornatum (Looss, 1900)

Gedoelst, 1903

Site: [intestine]

Host: Equus caballus

Dist.: Alta, Sask

Record: Ransom and Hadwen 1918

Cylicostephanus calicatus (Looss, 1900) Cram, 1924

Syn.: Cylicostomum calicatum (Looss, 1900)

Gedoelst, 1903

Site: [intestine]

Host: Equus caballus

Dist.: Alta, Sask

Record: Ransom and Hadwen 1918

Cylicostephanus goldi (Boulenger, 1917) Lichtenfels, 1975

Syn.: Cylicostomum goldi (Boulenger, 1917)

Ransom and Hadwen, 1918

Site: [intestine]

Host: Equus caballus

Dist.: Alta, Sask

Record: Ransom and Hadwen 1918

Cylicostephanus poculatus (Looss, 1900) Cram, 1924

Syn.: Cylicostomum poculatum Looss, 1900

Syn.: Petrovinema poculatum (Looss, 1900)

Ershov, 1943

Site: [intestine]

Host: Equus caballus

Dist.: Alta, Nfld, Sask

Records: Ransom and Hadwen 1918 (Alta,

Sask); Smith and Threlfall 1973 (Nfld)

Gyalocephalus capitatus Looss, 1900

Site: unspecified

Host: Equus caballus

Dist.: Alta, NB, Ont, Sask

Records: Ransom and Hadwen 1918 (Alta,

Sask); Swales 1933b (Ont); Smith 1976a (NB)

Gyalocephalus spp.

Site: unspecified

Host: Equus caballus

Dist.: NB

Record: Smith 1976a 
Oesophagodontus robustus (Giles, 1892) Railliet and Henry, 1902

Site: unspecified

Host: Equus caballus

Dist.: Alta, Nfld, Ont, Que, Sask

Records: Ransom and Hadwen 1918 (Alta,

Sask); Swales 1933b (Ont, Que); Threlfall 1969

(Nfld)

Oesophagodontus spp

Site: unspecified

Host: Equus caballus

Dist.: NB

Record: Smith 1976a

Poteriostomum spp.

Site: unspecified

Host: Equus caballus

Dist.: NB

Record: Smith 1976a

Strongylus edentatus (Looss, 1900) Railliet and Henry, 1909

Syn.: Alfortia edentatus (Looss, 1900) Skrjabin, 1933

Site: aorta

Host: Equus caballus

Dist.: Alta, NB, Nfld, Ont, Que, Sask

Records: Ransom and Hadwen 1918 (Alta);

Swales 1933a (Sask), 1933b (Que); Slocombe 1973a (Ont); Slocombe and McCraw 1973 (Ont); Smith and Threlfall 1973 (Nfld); Smith 1976a (NB), 1976b (NB)

Strongylus equinus Mueller, 1780

Site: unspecified

Host: Equus caballus

Dist.: Alta, NB, Ont, Que, Sask

Records: Ransom and Hadwen 1918 (Alta);

Swales 1933a (Sask), 1933b (Ont, Que);

Slocombe and McCraw 1973 (Ont); Smith

1976a (NB), 1976b exp. (NB)

Strongylus vulgaris (Looss, 1900) Railliet and Henry, 1909

Syn.: Delafondia vulgaris (Looss, 1900)

Skrjabin, 1933

Site: cranial mesenteric arteries, central nervous system

Host: Equus caballus

Dist.: Alta, NB, Nfld, Ont, Que, Sask

Records: Ransom and Hadwen 1918 (Alta, Sask); Swales 1933a (Sask); Little 1972 (Ont);

Slocombe 1973a (Ont); Smith and Threlfall

1973 (Nfld); Smith 1976a (NB), 1976b (NB);

Slocombe et al. 1987 (Ont)

Remarks: Little (1972) presumed the larva he recovered from the central nervous system of the horse was $S$. vulgaris.

Strongylus spp.

Syn.: Alfortia spp.

Site: unspecified

Host: Equus caballus

Dist.: NB, Ont

Records: Slocombe 1973a (Ont); Smith 1976a

(NB)
Triodontophorus brevicauda Boulenger, 1916

Site: unspecified

Host: Equus caballus

Dist.: Alta, Que, Sask

Records: Ransom and Hadwen 1918 (Alta,

Sask); Swales 1933b (Que)

Triodontophorus minor (Looss, 1900) Looss, 1902

Site: unspecified

Host: Equus caballus

Dist.: Nfld

Record: Smith and Threlfall 1973

Triodontophorus serratus (Looss, 1900) Looss, 1902 Syn.: Triodontophorus intermedius Sweet, 1909

Site: unspecified

Host: Equus caballus

Dist.: Alta, Nfld, Ont, Que, Sask

Records: Ransom and Hadwen 1918 (Alta, Sask); Swales 1933a (Sask), 1933b (Ont, Que); Smith and Threlfall 1973 (Nfld)

Triodontophorus tenuicollis Boulenger, 1916

Site: unspecified

Host: Equus caballus

Dist.: Alta, Sask

Record: Ransom and Hadwen 1918

Triodontophorus spp.

Site: unspecified

Host: Equus caballus

Dist.: NB, Ont

Records: Slocombe 1973a (Ont); Smith 1976a

(NB), 1976b (NB)

Unidentified Strongylidae

Site: unspecified

Host: Equus caballus

Dist.: NB, Ont, Que

Records: Swales 1933b (Ont, Que); Slocombe and McCraw 1973 (Ont); Smith 1976a (NB), 1976b (NB); Slocombe and Cote 1984 (Ont); Slocombe et al. 1987 (Ont)

\section{FAMILY SYNGAMIDAE}

Cyathostoma spp.

Site: [intestine]

Host: Equus caballus

Dist.: Nfld

Record: Threlfall 1969

Stephanurus dentatus Diesing, 1839

Site: kidney

Host: Sus scrofa

Dist.: Man, NS

Records: Swales 1933b (Man); Smith and

Hawkes 1978 (NS) 
SUPERFAMILY TRICHOSTRONGYLOIDEA

\section{FAMILY DICTYOCAULIDAE}

Dictyocaulus arnfieldi (Cobbold, 1884) Railliet and

Site: [lungs]

Henry, 1907

Host: Equus caballus

Dist.: Ont

Records: Swales 1933b

Dictyocaulus filaria (Rudolphi, 1809) Railliet and

Site: [lungs] Henry, 1907

Host: Ovis aries

Dist.: Alta, Canada, Nfld, Ont, PEI, Que

Records: Swales 1933b (Ont, PEI, Que); Fallis

1938 (Ont); Swales 1940a (Canada);

O'Donoghue 1958 (Alta); Smith and Threlfall

1973 (Nfld); Ayalew et al. 1973b (Que); Ayalew et al. 1974 (Que)

Dictyocaulus viviparus (Bloch, 1782) Railliet and

Site: lungs

$$
\text { Henry, } 1907
$$

Host: Bos taurus

Dist.: Alta, Nfld, Ont, Que

Records: Swales 1933b (Ont, Que); Campbell and Wetherill 1957 (Ont); Graesser 1957 (Alta);

O'Donoghue 1958 (Alta); Gupta and Gibbs

1969 (Que); Smith and Threlfall 1973 (Nfld);

Gupta and Gibbs 1975 (Que); Bateman et al.

1986 (Ont)

Dictyocaulus spp.

Site: [lungs]

Hosts: Bos taurus $(1,2)$

Dist.: Ont

Equus caballus (2)

Ovis aries (2)

Records: 1. McGregor and Kingscote 1957; 2.

Slocombe 1973a

\section{FAMILY HELIGMOSOMIDAE}

\section{Citellinema bifurcatum Hall, 1916}

Syn.: Warrenius bifurcatus (Hall, 1916) Sleggs, 1925

Site: unspecified

Host: Equus caballus

Dist.: Sask

Record: Swales 1933a

\section{FAMILY MOLINEIDAE}

Nematodirus abnormalis May, 1920

Site: unspecified

Host: Ovis aries

Dist.: Nfld

Record: Smith and Threlfall 1973

Nematodirus battus Crofton and Thomas, 1951

Site: [intestine]

Host: Ovis aries

Dist.: NS. PEI

Record: Smith and Hines 1987
Nematodirus filicollis Rudolphi, 1802

Site: [intestine]

Host: Ovis aries

Dist.: Canada, NS, Ont, Que

Records: Swales 1933b (Ont); Griffiths 1937

(Que); Fallis 1938 (Ont); Swales 1940a

(Canada), 1940b (Que); Swales and Choquette 1940 (Que); Smith and Archibald 1965 (NS);

Ayalew and Gibbs 1973 (Que)

Nematodirus helvetianus May, 1920

Site: [intestine]

Hosts: Bos taurus $(1,3,4,5,6,7)$

$$
\text { Ovis aries (2) }
$$

Dist.: NB, Ont

Records: 1. Campbell et al. 1960 (Ont); 2.

Smith and Archibald 1968 (NB); 3. Smith and Archibald 1969 (NB); 4. Smith 1970 (NB), 5.

1973 (NB); 6. Slocombe 1974b (Ont); 7 Smith 1974 (NB)

Nematodirus spathiger (Railliet, 1896) Railliet and Henry, 1909

Site: intestine

Hosts: Equus caballus (6) Ovis aries $(1,2,3,4,5,7,8,9)$

Dist.: Canada, Nfld, NS, Ont, Que

Records: 1. Swales 1933b (Ont); 2. Fallis 1938

(Ont); 3. Swales 1940a (Canada); 4. Swales and Choquette 1940 (Que); 5. Smith and Archibald 1965 (NS); 6. Webster and MacKay 1969 (Que); 7. Ayalew and Gibbs 1973 (Que); 8. Ayalew et al. 1973a (Que); 9. Smith and Threlfall 1973 (Nfld)

Nematodirus spp.

Site: unspecified

Hosts: Bos taurus $(3,4,6,8)$

Ovis aries $(1,2,5,7)$

Dist.: Ont, Que, Sask

Records: 1. Swales 1939 (Que); 2. Swales et al. 1942 (Ont); 3. McGregor and Kingscote 1957

(Ont); 4. Frechette and Gibbs 1971 (Que); 5.

Ayalew et al. 1973 a (Que); 6. Slocombe 1973b

(Ont); 7. Moteane et al. 1979 (Sask); 8. Polley

and Bickis 1987 (Sask)

Ollulanus tricuspis Leuckart, 1865

Site: stomach

Hosts: Felis catus $(1,3,4)$

Sus scrofa (2)

Dist.: Alta, Ont, Que

Records: 1. Cameron 1940b (Que); 2. Stockdale and Lautenslager 1973 (Ont); 3. Caldwell 1984

(Ont); 4. Kennedy et al. 1984 (Alta)

\section{FAMILY TRICHOSTRONGYLIDAE}

Cooperia curticei (Railliet, 1893) Ransom, 1907

Site: [intestine]

Host: Ovis aries

Dist.: Canada, NS, Ont, Que 
Records: Swales 1933b (Ont); Fallis 1938 (Ont); Swales 1940a (Canada), 1940b (Que); Swales and Choquette 1940 (Que); Smith and Archibald 1965 (NS); Ayalew and Gibbs 1973 (Que)

Cooperia mcmasteri

Site: [intestine]

Host: Bos taurus

Dist.: Ont

Record: Slocombe 1974b

Cooperia oncophora (Railliet, 1898) Ransom, 1907

Site: [intestine]

Hosts: Bos taurus $(5,6,7,9,10,11,12)$

Ovis aries $(1,2,3,4,8)$

Dist.: Canada, NB, Nfld, NS, Ont

Records: 1. Swales 1933b (Ont); 2. Fallis 1938 (Ont); 3. Swales 1940a (Canada); 4. Smith and Archibald 1965 (NS), 5. 1968 (NB); 6. Smith and Archibald 1969 (NB); 7. Smith 1970 (NB); 8. Ayalew and Gibbs 1973 (Que); 9. Smith 1973 (NB); 10. Smith and Threlfall 1973 (Nfld); 11. Slocombe 1974b (Ont); 12. Smith 1974 (NB)

Cooperia punctata (von Linstow, 1907) Ransom, 1907

Site: [intestine]

Host: Bos taurus

Dist.: Ont

Record: Swales 1933b

Cooperia spp.

Site: [intestine]

Hosts: Bos taurus $(3,5,7)$

Ovis aries $(1,2,4,6)$

Dist.: Alta, Ont, Que, Sask

Records: 1. Swales 1939 (Que); 2. Swales et al.

1942 (Ont); 3. Frechette and Gibbs 1971 (Que);

4. Ayalew et al. 1973a (Que); 5. Slocombe

1973a (Ont); 6. Moteane et al. 1979 (Sask); 7.

Stockdale and Harries 1979 (Alta)

Haemonchus contortus (Rudolphi, 1803) Cobb, 1898

Site: intestine

Hosts: Bos taurus $(1,7,8)$

Ovis aries $(1,2,3,4,5,6,9,10,11,12,13,14)$

Dist.: Canada, NS, Ont, Prairie Provinces, Que

Records: 1. Swales 1933b (Ont, Que); 2.

Griffiths 1937 (Que); 3. Fallis 1938 (Ont); 4.

Swales 1940a (Canada), 5. 1940b (Que); 6.

Swales and Choquette 1940 (Que); 7.

Choquette 1945 (Ont, Prairie Provinces, Que);

8. Johnston and MacPherson 1956 (Ont); 9.

Gibbs 1964 (Que); 10. Smith and Archibald

1965 (NS); 11. Blitz and Gibbs 1971 (Que); 12.

Ayalew and Gibbs 1973 (Que); 13. Ayalew et al.

1973a (Que); 14. Slocombe 1973c (Ont)

\section{Haemonchus spp.}

Site: [intestine]

Hosts: Bos taurus $(4,5,7)$

Ovis aries $(1,2,3,4,6)$

Dist.: Alta, Ont, Que, Sask
Records: 1. Swales 1933b (Ont), 2. 1939 (Que); 3. Swales et al. 1942 (Ont); 4. Slocombe 1973b (Ont), 5. 1974 (Ont); 6. Moteane et al. 1979

(Sask); 7. Stockdale and Harries 1979 (Alta)

Hyostrongylus rubidus (Hassall and Stiles, 1892) Hall, 1921

Site: stomach

Host: Sus scrofa

Dist.: Ont, Que

Records: 1. Swales 1933b (Ont); 2. Davenport and Stockdale 1967 (Ont); 3. Stockdale,

Ashton et al. 1973 (Ont); 4. Stockdale and

Lautenslager 1973 (Ont); 5. Martin et al. 1974

(Que)

Hyostrongylus spp.

Site: [stomach]

Host: Sus scrofa

Dist.: Ont

Record: Slocombe 1973a

Ostertagia circumcincta (Stadelmann, 1894) Ransom, 1907

Site: [intestine]

Hosts: Bos taurus (14)

Ovis aries $(1,2,3,4,5,6,7,8,9,10,11,12,13)$

Dist.: Canada, Maritimes, Nfld, NS, Ont, Que

Records: 1. Swales 1933b (Ont); 2. Griffiths

1937 (Ont); 3. Fallis 1938 (Ont); 4. Swales 1939

(Que), 5. 1940a (Canada), 6. 1940b (Que); 7.

Swales and Choquette 1940 (Que); 8. Swales et al. 1942 (Ont); 9. Smith and Archibald 1962 (Maritimes); 10. Smith and Archibald 1965 (NS); 11. Ayalew and Gibbs 1973 (Que); 12. Ayalew et al. 1973a (Que); 13. Swales and Threlfall 1973 (Nfld); 14. Slocombe 1974 (Ont)

Ostertagia lyrata Sjberg, 1926

Site: [intestine]

Host: Bos taurus

Dist.: Ont

Record: Slocombe 1974a, 1974b

Ostertagia ostertagi (Stiles, 1892) Ransom, 1907

Site: [intestine]

Host: Bos taurus

Dist.: NB, Ont, Que

Records: Swales 1933b (Que); Johnston and MacPherson 1956 (Ont); Smith and Archibald 1968 (NB); 1969 (NB); Smith 1970 (NB); Smith and Perreault 1972 (NB); Smith 1973 (NB); Slocombe 1974a (Ont), 1974b (Ont); Smith 1974 (NB)

Ostertagia trifurcata Ransom, 1907

Site: [intestine]

Hosts: Bos taurus $(3,5)$ Ovis aries $(1,2,4)$

Dist.: Alta, Maritimes, NS, Ont, Que

Records: 1. Smith and Archibald 1965 (NS); 2. Smith and Archibald 1962 (Maritimes); 3. Slocombe 1970 (Alta); 4. Ayalew and Gibbs 1973 (Que); 5. Slocombe 1974b (Ont)

Remarks: In Slocombe (1970), the ox originated in Alberta and was slaughtered in Ontario. 
Ostertagia spp.

Site: [intestine]

Hosts: Bos taurus $(3,6,8)$

Ovis aries $(1,2,4,5,6,7)$

Dist.: Alta, Ont, Que, Sask

Records: 1. Swales 1940b (Que); 2. Gibbs 1964 (Que); 3. Frechette and Gibbs 1971 (Que); 4.

Ayalew and Gibbs 1973 (Que); 5. Ayalew et al. 1973a (Que); 6. Slocombe 1973b (Ont); 7.

Moteane et al. 1979 (Sask); 8. Stockdale and

Harries 1979 (Alta)

Teladorsagia davtiani Andreeva and Satubaldin, 1954

Site: [intestine]

Host: Ovis aries

Dist.: Maritimes, NS

Records: Smith and Archibald 1962

(Maritimes), 1965 (NS)

Trichostrongylus axei (Cobbold, 1879) Railliet and Henry, 1909

Syn.: Trichostrongylus extenuatus (Railliet, 1898) Ransom, 1907

Site: [intestine]

Hosts: Bos taurus $(10,11,14,15)$ Ovis aries $(1,2,3,4,5,6,7,8,9,12,13,14)$

Dist.: Canada, NB, NS, Ont, Que

Records: 1. Swales 1933b (Ont), 2. 1939 (Que);

3. Fallis 1938 (Ont); 4. Swales 1940a (Canada),

5. 1940b (Que); 6. Swales and Choquette 1940

(Que); 7. Swales et al. 1942 (Ont); 8. Gibbs 1964

(Que); 9. Smith and Archibald 1965 (NS); 10.

Smith 1970 (NB); 11. Frechette and Gibbs 1971 (Que); 12. Ayalew and Gibbs 1973 (Que); 13.

Ayalew et al. 1973a (Que); 14. Slocombe 1973a

(Ont), 15. 1974 (Ont)

Trichostrongylus colubriformis (Giles, 1892)

Site: [intestine]

Ransom, 1911

Host: Ovis aries

Dist.: Canada, NS, Ont, Que

Records: Swales 1933a (Ont); Griffiths 1937

(Que); Fallis 1938 (Ont); Swales 1939 (Que),

1940a (Canada), 1940b (Que); Swales and

Choquette 1940 (Que); Smith and Archibald

1965 (NS); Ayalew and Gibbs 1973 (Que)

Trichostrongylus vitrinus Looss, 1905

Site: [intestine]

Host: Ovis aries

Dist.: Canada, NS, Ont, Que

Records: Swales 1933a (Ont); Fallis 1938 (Ont);

Swales 1939 (Que), 1940a (Canada); Swales

and Choquette 1940 (Que); Smith and

Archibald 1965 (NS); Ayalew and Gibbs 1973

(Que)

Trichostrongylus spp.

Site: [intestine]

Hosts: Bos taurus $(6,8)$

Ovis aries $(1,2,3,4,5,6,7)$

Dist.: Alta, Ont, Que, Sask
Records: 1. Griffiths 1937 (Que); 2. Swales 1939 (Que); 3. Swales et al. 1942 (Ont); 4. Ayalew and Gibbs 1973 (Que); 5. Ayalew et al. 1973a (Que); 6. Slocombe 1973b (Ont); 7. Moteane et al. 1979 (Sask); 8. Stockdale and Harries 1979 (Alta)

Unidentified Trichostrongyloidea (egg)

Site: [intestine]

Host: Bos taurus

Dist.: Ont

Record: McGregor and Kingscote 1957

Unidentified Nematoda

Site: unspecified

Hosts: Bos taurus $(2,4)$

Canis familiaris (3)

Equus caballus (1)

Dist.: Nfld, Que, Sask

Records: 1. Threlfall 1969 (Nfld); 2. Frechette and Gibbs 1971 (Que); 3. Olson 1985 (Sask); 4.

Polley and Bickis 1987 (Sask)

\section{PHYLUM ACANTHOCEPHALA}

CLASS ARCHIACANTHOCEPHALA

\section{ORDER OLIGACANTHOR HYNCHIDA}

\section{FAMILY OLIGACANTHOR HYNCHIDAE}

Macracanthorhynchus hirudinaceus (Pallas, 1781)

Site: [intestine]

Host: Sus scrofa

Dist.: Ont

Record: Swales 1933b

CLASS PALAEACANTHOCEPHALA

ORDER POLYMORPHIDA

FAMILY POLYMORPHIDAE

Corynosoma semerme (Forssell, 1904)

Site: [intestine]

Host: Canis familiaris

Dist.: NWT

Record: Cameron et al. 1940

PHYLUM CHELICERATA

CLASS ARACHNIDA

SURCLASS ACARI

ORDER ACARIFORMES

SUBORDER ACARIDIDA

FAMILY PSOROPTIDAE

Chorioptes bovis (Hering, 1845) Gervais and Van

Beneden, 1859

Site: unspecified

Hosts: Bos taurus $(1,2,3)$

Equus caballus (1)

Dist.: Alta, BC, Ont 
Record: 1. Slocombe 1973a (Ont); 2. Lavigne and Smith 1983 (Alta, BC); 3. Kennedy and Kralka 1986 (Alta)

Remarks: Slocombe (1973a) recorded the mite as Chorioptes spp.

Otodectes cynotis (Hering, 1838) Canestrini, 1894

Site: ear

Hosts: Canis familiaris (2)

Felis catus $(1,2,3)$

Dist.: BC, NB, Que

Records: 1. Spencer 1940 (BC); 2. Sweatman 1958 (Que); 3. Malloy and Embil 1978 (NB)

Otodectes cynotis canis Sewell

Host: Canis familiaris

Dist.: BC

Record: Spencer 1939

Otodectes cynotis felis Huber

Host: Felis catus

Dist: BC

Record: Spencer 1939

Psoroptes spp.

Site: unspecified

Host: Equus caballus

Dist.: Ont

Record: Slocombe 1973a

FAMILY SARCOPTIDAE

Notoedres spp.

Site: unspecified

Host: Canis familiaris

Dist.: Ont

Record: Slocombe 1973a

Sarcoptes scabiei (Linnaeus, 1758)

Site: unspecified

Hosts: Bos taurus $(2,3,5)$

Canis familiaris (2)

Felis catus (1)

Sus scrofa $(2,4,6)$

Dist.: Alta, BC, NB, Ont, Que

Record: 1. Tyrrell 1883 (unspecified); 2.

Slocombe 1973a (Ont); 3. Lavigne and Smith 1983 (Alta, BC); 4. Martineau et al. 1984 (Que); 5. Kennedy and Kralka 1986 (Alta); 6. Smith 1986 (NB)

\section{SUBORDER ACTINEDIDA}

\section{FAMILY CHEYLETIDAE}

Cheyletiella spp.

Site: unspecified

Host: Canis familiaris

Dist.: Ont, Sask

Record: Slocombe 1973a (Ont); Olson 1985

(Sask)

\section{FAMILY DEMODICIDAE}

Demodex bovis Stiles, 1892

Site: [skin]

Host: Bos taurus

Dist.: Alta, Ont

Record: Smith 1961 (Ont); Kennedy and

Kralka 1986 (Alta)

Demodex phylloides Croker

Host: Sus scrofa

Dist.: Ont

Records: Jarvis 1910; MacNay 1952

Remarks: MacNay (1952) felt his specimens were probably $D$. phylloides.

Demodex spp.

Site: unspecified

Hosts: Bos taurus

Dist.: Ont Canis familiaris

Record: Slocombe 1973a

\section{FAMILY PSORERGATIDAE}

Psorergates bos Johnston, 1964

Site: [skin]

Host: Bos taurus

Dist.: Alta, unspecified

Records: Lavigne and Smith 1983

(unspecified); Kennedy and Kralka 1986 (Alta)

\section{FAMILY TROMBICULIDAE}

Unidentified Trombiculidae

Site: unspecified

Host: Canis familiaris

Dist.: Ont

Record: Slocombe 1973a

ORDER PARASITIFORMES

SUBORDER GAMASIDA

FAMILY DERMANYSSIDAE

Pneumonyssus spp.

Site: Unspecified

Host: Canis familiaris

Dist.: Ont

Record: Slocombe 1973a

\section{SUBORDER IXODIDA}

\section{FAMILY ARGASIDAE}

Otobius lagophilus Cooley and Kohls, 1940

Site: unspecified

Host: Felis catus

Dist.: Alta

Record: Brown 1944b 
Otobius megnini (Duges, 1883) Banks, 1912

Site: unspecified

Hosts: Bos taurus $(2,3)$

Felis catus $(1,3)$

Ovis aries (2)

Dist.: BC

Record: 1. Gregson 1953; 2. MacNay 1957; 3.

Rich 1957

Otobius spp.

Site: unspecified

Host: Canis familiaris

Dist.: Ont

Record: Slocombe 1973a

\section{FAMILY IXODIDAE}

Dermacentor albipictus (Packard, 1869) Banks, 1908

Syn.: Dermacentor erraticus var. albipictus

(Packard)

Site: skin

Hosts: Bos taurus $(1,2,4,5,9)$

Canis familiaris (8)

Equus caballus $(1,2,3,4,5,6,7,8)$

Dist.: Alta, BC, NB, NS, Sask

Records: 1. Hewitt 1915 (BC, NB); 2. Cameron and Fulton 1927 (Sask); 3. Hearle 1933 (BC), 4. 1938 (BC); 5. Bequaert 1945 (NB, NS); 6.

Brown and Kohls 1950 (Alta); 7. MacNay 1952 (BC), 8. 1957 (Sask); 9. Wilkinson and Lawson 1965 (unspecified)

Dermacentor andersoni Stiles, 1905

Syn.: Dermacentor venustus Banks, 1908

Site: skin

Hosts: Bos taurus $(3,7,10,11,12,14,15,17,19,20)$

Canis familiaris $(6,13,18)$

Equus caballus $(2,6,7,13)$

Felis catus (13)

Ovis aries $(1,4,5,6,7,8,9,11,13)$

Sus scrofa (13)

unspecified (12)

Dist.: Alta, BC, Sask

Records: 1. Hadwen 1913 (BC); 2. Hewitt 1915

(Alta, BC); 3. Hearle 1933 (BC); 4. Gregson 1935 (BC), 5. 1937 (BC); 6. Hearle 1938 (BC); 7. Holland 1940 (Alta); 8. Gregson 1947 (BC), 9. 1952 (BC); 10. MacNay 1952 (BC, Sask); 11.

Weintraub 1952 (BC); 12. MacNay 1957 (BC);

13. Gregson 1958a (BC); 14. Gregson 1958c

(BC); 15. Wilkinson and Lawson 1965 (Alta,

BC); 16. Wilkinson 1968 (BC), 17. 1972 (Alta,

BC, Sask); 18. Burt et al. 1979 (BC); 19.

Wilkinson 1981 (Alta, BC), 20. 1982 (BC)

Dermacentor variabilis (Say, 1821) Banks, 1908

Site: unspecified

Host: Canis familiaris $(1,2,3)$

collected by flagging (4)

Dist.: Man, NS, Ont

Records: 1. Bequaert 1945 (Ont); 2. MacNay

1952 (Man); 3. Judd 1953 (Ont); 4. Hall and

McKiel 1961 (NS)
Dermacentor spp.

Site: unspecified

Hosts: Felis catus

Dist.: Ont Equus caballus

Record: Slocombe 1973a

Haemaphysalis cinnabarina Koch

Host: Bos taurus

Dist.: Man

Record: Hewitt 1915

Haemaphysalis leporis-palustris (Packard)

Host: Felis catus

Dist.: BC

Record: Hearle 1938

Ixodes ccokei Packard, 1869

Syn.: Ixodes hexagonus cookei Packard, 1869

Hosts: Bos taurus (3)

Canis familiaris $(1,2,3,4)$

Felis catus $(3,5)$

Dist.: BC, NB, Ont, Que

Records: 1. Hewitt 1915 (Ont); 2. Hearle 1938

(BC); 3. Bequaert 1945 (NB, Ont, Que); 4.

Cooley and Kohls 1945 (Ont); 5. Judd 1954

(Ont)

Ixodes ricinus Linnaeus

Host: Canis familiaris

Dist.: BC

Records: Hewitt 1915; Hearle 1938

Ixodes ricinus scapularis Say

Host: Canis familiaris

Dist.: $\mathrm{BC}$

Record: Hearle 1938

Ixodes spp.

Site: unspecified

Hosts: Canis familiaris

Felis catus

Dist.: Ont

Record: Slocombe 1973a

Rhipicephalus sanguineus (Latreille, 1806) Koch, 1844

Site: unspecified

Host: Canis familiaris

Dist.: BC, NS, Ont

Record: Bequaert 1945 (NS, Ont); MacNay

1957 (Ont); Spencer 1960 (BC); Wright 1979

(NS)

\section{Rhipicephalus spp.}

Site: unspecified

Host: Canis familiaris

Dist.: Ont

Record: Slocombe 1973a 


\section{PHYLUM UNIRAMIA}

CLASS INSECTA

ORDER DIPTERA

FAMILY CALLIPHORIDAE

Calliphora spp.

Host: unspecified

Dist.: BC

Record: MacNay 1952

Lucilia illustris Meigen, 1826

Site: unspecified

Host: Ovis aries

Dist.: Ont

Record: Teskey 1960

Lucilia sericata (Meigen, 1826)

Syn.: Phaenicia sericata (Meigen, 1826)

Host: Ovis aries

Dist.: Nfld

Record: MacNay 1957

Phormia regina Meigen, 1826

Site: unspecified

Hosts: Bos taurus (1)

Ovis aries (2)

Dist.: BC, Ont

Records 1. Hearle 1933 (BC); 2. Teskey 1960 (Ont)

Phormia terranovae Robineau-Desvoidy, 1830

Syn.: Protophormia terranovae (Robineau-

Desvoidy, 1830) Townsend, 1908

Site: unspecified

Host: Ovis aries

Dist.: Ont

Record: Teskey 1960

Wohlfahrtia opaca (Coquillet)

Site: unspecified

Host: unspecified

Dist.: BC

Record: MacNay 1952

Wohlfahrtia vigil (Walker, 1949)

Site: unspecified

Host: unspecified

Dist.: BC

Record: MacNay 1952

Unidentified Calliphoridae

Site: unspecified

Host: Felis catus

Dist.: Ont

Record: Slocombe 1973a

\section{FAMILY CUTEREBRIDAE}

\section{Cuterebra spp.}

Site: unspecified

Hosts: Canis familiaris (2)

Dist.: Ont

$$
\text { Felis catus }(1,2)
$$

Records: 1. Scholten et al. 1962; 2. Slocombe 1973a

\section{FAMILY GASTEROPHILIDAE}

Gasterophilus intestinalis (De Geer, 1776) Leach, 1817

Site: mouth

Host: Equus caballus $(2,3,4,5)$ unspecified (1)

Dist.: Alta, Ont, Sask

Records: 1. MacNay 1952 (Sask); 2. Nelson 1952 (Alta); 3. Teskey 1960 (Ont); 4. Slocombe 1973a (Ont); 5. Slocombe et al. 1987 (Ont)

Gasterophilus nasalis (Linnaeus, 1758) Leach, 1817

Site: mouth

Host: Equus caballus

Dist.: Alta, Ont

Records: Nelson 1952 (Alta); Teskey 1960

(Ont); Slocombe 1973a (Ont); Slocombe et al. 1987 (Ont)

Gasterophilus spp.

Host: Equus caballus

Dist.: Man

Record: MacNay 1952

\section{FAMILY HIPPOBOSCIDAE}

Melophagus ovinus (Linnaeus, 1758) Latreille, 1802

Site: skin

Host: Ovis aries

Dist.: BC, Ont, Sask

Records: Spencer 1938 (BC), 1939 (BC);

MacNay 1952 (BC); Teskey 1960 (Ont);

Slocombe 1973a (Ont); Moteane et al. 1979 (Sask)

\section{FAMILY OESTRIDAE}

Hypoderma bovis (Linnaeus, 1758) Latreille, 1818

Site: subcutaneous tissues

Host: Bos taurus

Dist.: Alta, BC, Man, Ont, Que, Sask

Records: Gregson 1958b (Alta, BC); Teskey

1960 (Ont); Murray 1967 (Man); Clermont and

Ruppanner 1975 (Que); Khan 1981 (Alta);

Galloway and Harlos 1982 (Man, Sask, Alta)

Hypoderma lineatum (Villers, 1789) Latreille, 1818

Site: subcutaneous tissues

Host: Bos taurus

Dist.: Alta, BC, Man

Records: Gregson 1958b (Alta, BC); Murray

1967 (Man); Nelson and Weintraub 1972

(Alta); Khan 1981 (Alta)

Hypoderma spp.

Site: oesophagus, subcutaneous tissues

Hosts: Bos taurus $(2,3)$

Equus caballus (2)

unspecified (1)

Dist.: Alta, BC, Man, Ont, Sask

Records: 1. MacNay 1957 (Alta, BC, Man,

Ont); 2. Slocombe 1973a (Ont); 3. Blakley 1979 (Sask) 
Oestrus ovis Linnaeus, 1761

Site: unspecified

Host: Ovis aries

Dist.: Ont, Western Canada

Records: Fallis 1940 (Ont, Western Canada);

Teskey 1960 (Ont); Slocombe 1973a (Ont)

Unidentified Diptera

Site: lung

Host: Ovis aries

Dist.: BC

Record: MacNay 1952

Remarks: One rat-tailed maggot found in the lung of a sheep (MacNay 1952).

\section{ORDER MALLOPHAGA}

\section{SUBORDER ISCHNOCERA}

Damalinia bovis (Linnaeus, 1758) Mjoberg, 1910

Syn.: Bovicola bovis

Site: unspecified

Host: Bos taurus

Dist.: Alta, BC, Man, NS, Ont, Que

Record: Thompson 1934 (NS, Que);

Whitehead 1934 (Que); Spencer 1939 (BC);

MacNay 1952 (Man); Hopkins 1960 (BC);

Teskey 1960 (Ont); Lavigne and Smith 1983

(Alta, BC); Kennedy and Kralka 1986 (Alta)

Remarks: Lyal (1987) classified this louse as

Bovicola bovis.

Damalinia caprae (Gurlt, 1843)

Syn.: Bovicola caprae (Gurlt, 1843)

Host: Capra hircus

Dist.: BC

Record: Spencer 1939

Remarks: According to Lyal (1987) this is

Bovicola caprae.

Damalinia equi (Denny, 1842)

Syn: Trichodectes equi (Linnaeus)

Site: [skin]

Host: Equus cabalus

Dist.: BC

Record: Spencer 1939; Hopkins 1960

Remarks: According to Lyal (1987) this is

Werneckiella equi.

Damalinia limbata (Gervais, 1844) Mjoberg, 1910

Site: unspecified

Host: Capra hircus

Dist.: Ont

Record: Teskey 1960

Remarks: According to Lyal (1987) this is

Bovicola limbatus.

Damalinia spp.

Site: unspecified

Hosts: Bos taurus

Dist.: Ont Equus caballus

Record: Slocombe 1973a
Felicola subrostratus (Nitzsch, 1838)

Site: [skin]

Host: Felis catus

Dist.: BC, Que

Records: Whitehead 1934 (Que); Hopkins 1960 (BC)

Trichodectes canis (de Geer, 1778)

Site: [skin]

Host: Canis familiaris

Dist.: BC

Records: Spencer 1939; Hopkins 1960

Trichodectes spp.

Site: unspecified

Host: Canis familiaris

Dist.: Ont

Record: Slocombe 1973a

ORDER SIPHONAPTERA

FAMILY CERATOPHYLLIDAE

Monopsyllus vison (Baker, 1904)

Site: [skin]

Host: Felis catus

Dist.: Sask

Record: Holland 1984

\section{FAMILY HYSTRICHOPSYLLIDAE}

Tamiophila grandis (Rothschild, 1902)

Site: [skin]

Host: Canis familiaris

Dist.: NB

Record: Brown 1968

\section{FAMILY PULICIDAE}

Ctenocephalides canis (Curtis, 1826)

Hosts: Canis familiaris $(1,2,3,4,5,6)$

Felis catus $(3,4)$

Dist.: Alta, BC, NB, Ont

Records: 1. Harvey 1907 (BC); 2. Spencer 1936

(BC), 3. 1939 (BC); 4. Brown 1944a (Alta); 5.

Brown 1955 (NB); 6. Holland 1984 (BC, Ont)

Ctenocephalides felis (Bouche, 1835)

Hosts: Canis familiarus $(2,3)$

Felis catus $(1,2,3)$

Dist.: BC, NS

Records: 1. Spencer 1936 (BC), 2. 1939 (BC); 3.

Wright 1979 (NS)

Ctenocephalides felis felis (Bouche, 1835)

Site: unspecified

Hosts: Canis familiaris $(2,3,4)$

Felis catus $(1,2,3,4)$

Dist.: Alta, BC, Man, Nfld, NB, NS, Ont, Que, Sask

Records: 1. Brown 1944a (Alta); 2. Brown 1955 (NB, NS); 3. Buckner 1964 (Man); 4. Holland 1984 (BC, Man, Nfld, NS, Ont, Que, Sask) 
Ctenocephalides spp.

Site: unspecified

Hosts: Felis catus (3) unspecified $(1,2)$

Dist.: Canada, Ont, PEI

Records: 1. MacNay 1952 (Ont, PEI), 2. 1957

(Canada); 3. Slocombe 1973a (Ont)

Remarks: MacNay (1957) refered to

Ctenocephalides spp. as infesting household pets from coast to coast.

\section{ORDER SIPHUNCULATA}

\section{FAMILY HAEMATOPINIDAE}

Haematopinus asini (Linnaeus, 1775)

Host: Equus caballus

Dist.: BC, Que

Records: Whitehead 1934 (Que); Spencer 1939

(BC), 1966 (BC)

Haematopinus eurysternus (Nitzsch, 1818)

Site: unspecified

Host: Bos taurus

Dist.: Alta, BC, NS, Ont, Que

Records: Thompson 1934 (NS, Que);

Whitehead 1934 (Que); Spencer 1939 (BC);

Teskey 1960 (Ont); Spencer 1966 (BC); Lavigne and Smith 1983 (Alta, BC); Khan and Schaalje 1985 (Alta)

Haematopinus suis (Linnaeus, 1758) Leach, 1815

Site: unspecified

Host: Sus scrofa

Dist.: BC, Ont, Que

Records: Whitehead 1934 (Que); Spencer 1939

(BC); Rich 1952 (BC); Teskey 1960 (Ont);

Spencer 1966 (BC); Wright 1979 (Que)

Haematopinus spp.

Site: unspecified

Hosts: Bos taurus

Equus caballus

Dist.: Ont

Sus scrofa

Record: Slocombe 1973a

\section{FAMILY LINOGNATHIDAE}

Linognathus pedalis (Osborn, 1896)

Site: [skin]

Host: Ovis aries

Dist.: BC

Record: Spencer 1939

Linognathus setosus (Olfers, 1816)

Syn.: Linognathus piliferus (Burmeister)

Site: [skin]

Host: Canis familiaris

Dist.: BC, Man, Ont, Que

Records: Whitehead 1934 (Que); Spencer 1939

(BC); MacNay 1952 (Man, Ont); Judd 1953

(Ont)
Linognathus stenopsis (Burmeister, 1839) Enderlein, 1905

Site: unspecified

Host: Capra hircus

Dist.: Ont

Record: Teskey 1960

Linognathus vituli (Linnaeus, 1758) Enderlein, 1905

Site: unspecified

Host: Bos taurus

Dist.: Alta, BC, Ont, Que

Records: Whitehead 1934 (Que); Teskey 1960

(Ont); Lavigne and Smith 1983 (Alta, BC);

Kennedy and Kralka 1986 (Alta)

Linognathus spp.

Site: unspecified

Hosts: Bos taurus

Dist.: Ont Canis familiaris

Record: Slocombe 1973a

Solenopotes capillatus Enderlein, 1904

Site: unspecified

Host: Bos taurus

Dist.: Alta, Ont

Records: Teskey 1960 (Ont); Kennedy and

Kralka 1986 (Alta)

Unidentified lice

Site: [skin]

Host: Bos taurus

Dist.: BC, Man

Record: MacNay 1957 


\section{HOST-PARASITE LIST}



CLASS MAMMALIA

\section{INFRACLASS EUTHERIA}

\section{ORDER ARTIODACTYLA}

FAMILY BOVIDAE

Bostaurus $\mathrm{Ox}$

PROTOZOA

Coccidia gen. sp. (Ont)

Cryptosporidium spp. (Man, Ont)

Eimeria alabamanensis (Alta, Ont)

E. arloingi (Ont)

E. aubernensis (Alta, Ont)

E. bovis (Alta, Ont)

E. bukidnonensis (Ont)

E. canadensis (Alta, Ont)

E. cylindrica (Alta, Ont)

E. ellipsodalis (Alta, Ont)

E. illinoisensis (Alta)

E. wyomingensis (Alta, Ont)

E. zuernii (Alta, Ont)

Eimeria sp. (Alta)

Giardia spp. (Sask)

Sarcocystis cruzi (Sask)

Sarcocystis hirsuta (Sask)

Sarcocystis (Ont, Sask)

Trichomonas spp. (Ont)

Toxoplasma gondii(Ont)

Toxoplasma spp. (Que)

Trypanosoma rutherfordi $(\mathrm{BC})$

T. theileri (Ont)

T. uniforme (Ont)

Trypanosoma spp. (BC)

\section{TREMATODA}

Calicophoron microbothrioides (Que)

Dicrocoelium dendriticum (BC, Nfld)

Fasciola hepatica (Que)

Fascioloides magna (Alta, Ont)

Paramphistomum liorchis (Que)

Paramphistomum spp. (Alta, Ont)

\section{CESTOIDEA}

Moniezia benedeni egg (NB, Que)

M. expansa (Que)

Moniezia spp. (Ont, Que)

Taenia hydatigena (Ont, Que)

Taeniarhyncus saginatus (Ont, Sask)

\section{NEMATODA}

Ascaris lumbricoides larva (Ont)

Ascaris suum (Ont)

Bunostomum phlebotomum (Que)

B. trigonocephalum (Ont, Que)

Bunostomum spp. (Ont, Que)

Capillaria spp. (Ont, Que)

Cooperia mcmasteri (Ont)

Cooperia oncophora (NB, Nfld)
C. punctata (Ont)

Cooperia spp. (Alta, Ont, Que)

Dictyocaulus viviparus (Alta, Nfld, Ont, Que)

Dictyocaulus spp. (Ont)

Haemonchus contortus (Ont, Prairie

Provinces, Que)

Haemonchus spp. (Alta, Ont)

Nematoda gen. sp. (Que)

Nematodirus helvetianus (NB, Ont)

Nematodirus spp. (Ont, Que)

Oesophagostomum radiatum (NB, Nfld, Ont,

Que)

Oesophagostomum spp. (Ont, Que)

Onchocerca gutturosa (Ont, Que)

O. lienalis (Ont, Que)

Onchocerca spp. (Man, Sask)

Ostertagia circumcincta (Ont)

O. lyrata (Ont)

O. ostertagi (NB, Ont, Que)

O. trifurcata (Alta, Ont)

Ostertagia spp. (Alta, Ont, Que)

Parafilaria bovicola (Alta, Que)

Setaria labiatopapillosa (Alta, Man, Ont,

Que)

Stephanofilaria stilesi (Alta)

Strongyloidea gen. sp. (Ont)

Strongyloides papillosus (Que)

Strongyloides spp. (Ont)

Thelazia gulosa (Ont)

T. lacrymalis (Ont)

T. skrjabini (Alta)

Thelazia spp. (Que)

Trichostrongyloidea gen. sp. egg (Ont)

Trichostrongylus axei (NB, Ont, Que)

Trichostrongylus spp. (Alta, Ont)

Trichuridae gen. sp. (Ont)

Trichuris discolor (Ont, NB, Que)

Trichuris ovis (NB, Que)

Trichuris spp. (Alta, NB, NWT, Ont, Sask)

Trichuris spp. egg (NB, Ont)

Unidentified Nematoda (Que, Sask)

Unidentified Rhabditoidea (Ont)

\section{CHELICERATA}

ACARI

Chorioptes bovis (Alta, BC, Ont)

Demodex bovis (Alta, Ont)

Demodex spp. (Ont)

Dermacentor andersoni (Alta, BC, Sask)

D. albipictus (BC, NB, NS, Sask)

Haemaphysalis cinnabarina (Man)

Ixodes cookei (NB, Ont, Que)

Otobius megnini (BC)

Psorergates bos (Alta)

Sarcoptes scabiei (Alta, BC, Ont)

\section{UNIRAMIA}

DIPTERA

Hypoderma bovis larva (Alta, BC, Man, Ont, Que, Sask) 
H. lineatum (Alta, BC, Man)

Hypoderma spp. (Ont, Sask)

Phormia regina $(\mathrm{BC})$

\section{MALLOPHAGA}

Damalinia bovis (Alta, BC, Man, NS, Ont, Que)

Damalinia spp. (Ont)

SIPHUNCULATA

Haematopinus eurysternus (Alta, BC, NS,

Ont, Que)

Haematopinus spp. (Ont)

Lice (unidentified) (BC, Man)

Linognathus vituli (Alta, BC, Ont, Que)

Linognathus spp. (Ont)

Solenopotes capillatus (Alta, Ont)

Caprahircus Domestic Goat

PROTOZOA

Toxoplasma gondii(Ont)

\section{NEMATODA}

Muellerius capillaris (Ont)

Muellerius spp. (Ont)

UNIRAMIA

MALLOPHAGA

Damalinia caprae (BC)

D. limbata(Ont)

SIPHUNCULATA

Linognathus stenopsis (Ont)

\section{Ovis aries Domestic Sheep}

PROTOZOA

Coccidia gen. sp. (Que, Sask)

Eimeria ahsata (Alta, Sask)

E. arloingi (Alta, Ont)

E. crandallis (Alta)

E. faurei (Alta)

E. granulosa (Alta)

E. intricata (Alta)

E. ninakohlyakimovae (Alta, Ont, Sask)

E. ovina (Sask)

E. pallida (Alta)

E. parva (Alta, Ont, Sask)

E. punctata (Alta)

Eimeria spp. (Que)

Sarcocystis spp. (Sask)

Toxoplasma gondii(Ont)

Toxoplasma spp. (Ont, Que)

\section{TREMATODA}

Dicrocoelium dendriticum (NS, Que)

Fasciola hepatica (Canada, BC, NB, Que)

\section{CESTOIDEA}

Moniezia expansa (Canada, Nfld, NS, Ont, Que)
Moniezia spp. (Nfld, Sask)

Taenia hydatigena cysticercus (Alta, Canada, Ont, Que, Sask)

T. ovis (Canada, Man, NS)

Thysanosoma actinioides (Alta, BC, Canada, Sask)

Thysanosoma spp. (Ont, Sask)

\section{NEMATODA}

Ascaris suum (Que)

Ascaris spp. (Canada, Ont)

Bunostomum trigonocephalum (Canada,

Nfld, NS, Ont, Que)

Bunostomum spp. (Que, Sask)

Capillaria longipes (Canada, Que)

Capillaria spp. (NS, Ont, Que)

Chabertia ovina (Canada, NS, Ont, Que)

Chabertia spp. (Ont, Sask)

Cooperia curticei (Canada, NS, Ont, Que)

C. oncophora (Canada, NS, Ont, Que)

Cooperia spp. (Ont, Que, Sask)

Dictyocaulus filaria (Alta, Canada, Nfld, Ont,

PEI, Que)

Dictyocaulus spp. (Ont)

Gongylonema pulchrum (Canada, Ont)

Haemonchus contortus (Canada, NS, Ont, Que)

Haemonchus spp. (Ont, Que, Sask)

Muellerius capillaris (Canada, Ont)

Muellerius spp. (Ont)

Nematodirus abnormalis (Nfld)

N. battus (NS, PEI)

$N$. filicollis (Canada, NS, Ont, Que)

$N$. helvetianus (NB, Ont)

$N$. spathiger (Canada, Nfld, NS, Ont, Que)

Nematodirus spp. (Ont, Que, Sask)

Oesophagostomum columbianum (Canada, NS, Ont, Que)

O. venulosum (Canada, Nfld, NS, Ont, Que)

Oesophagostomum spp. (Nfld, Ont, Que)

Ostertagia circumcincta (Canada, Maritimes,

Nfld, NS, Ont, Que)

O. trifurcata (Maritimes, NS, Que)

Ostertagia spp. (Ont, Que, Sask)

Parelaphostrongylus tenuis (Ont)

Protostrongylus spp. (Ont)

Skrjabinema spp. (Sask)

Strongyloides papillosus (Canada, NS, Ont, Que)

Strongyloides spp. (Ont, Que, Sask)

Telodorsagia davtiani (Maritimes, NS)

Trichostrongylus axei (Canada, NS, Ont, Que)

T. colubriformis (Canada, NS, Ont, Que)

T. vitrinus (Canada, NS, Ont, Que)

Trichostrongylus spp. (Ont, Que, Sask)

Trichuris ovis (Canada, Nfld, NB, NS, Ont, Que) 
Trichuris spp. (Alta, NWT, Ont, Sask)

\section{CHELICERATA \\ ACARINA}

Dermacentor andersoni (Alta, BC)

Otobius megnini (BC)

\section{UNIRAMIA \\ DIPTERA}

Lucilia illustris (Ont)

L. sericata $(\mathrm{Nfld})$

Melophagus ovinus (BC, Ont, Sask)

Oestrus ovis (Ont, Western Canada)

Phormia regina (Ont)

P. terranovae (Ont)

Unidentified Diptera (BC)

SIPHUNCULATA

Linognathus pedalis $(\mathrm{BC})$

\section{FAMILY SUIDAE}

Sus scrofa Domestic Pig

PROTOZOA

Balantidium spp. (Ont)

Cryptosporidium spp. (Ont)

Eimeria dublieki (Ont, Que)

Isospora suis (Ont, Que)

Toxoplasma gondii (Ont)

Toxoplasma spp. (Ont)

Trichomonas spp. (Ont)

Unidentified Eucoccidiida (Que)

TREMATODA

Paragonimus spp. (Ont)

\section{CESTOIDEA}

Echinococcus granulosus hydatid cyst (Ont)

Echinococcus spp. hydatid (Ont)

Multiceps spp. (Ont)

Taenia hydatigena (Que)

T. solium (Man, Ont, Sask)

NEMATODA

Ascaris lumbricoides larva (All Provinces,

Ont)

A. suum (Nfld, Que, Sask)

Ascaris spp. (Ont)

Ascarops spp. (Ont)

Hyostrongylus rubidus (Ont, Que)

Hyostrongylus spp. (Ont)

Metastrongylus apri (NB)

M. pudendotectus (Ont)

M. salmi (NS, Ont, PEI, Que)

Metastrongylus spp. (Ont)

Oesophagostomum dentatum (NB, Ont, Que)

$O$. quadrispinulatum (NB)

Oesophagostomum spp. (NB, Ont, Que)

Ollulanus tricuspis (Ont)

Physocephalus spp. (Ont)
Stephanurus dentatus (Man, NS)

Strongyloides ransomi (NB)

Strongyloides spp. (Ont)

Trichinella spiralis cyst/ larva (BC, Man, NB,

NS, Ont, PEI, Que)

T.s. spiralis (NS)

Trichinella spp. (NB)

Trichuris suis (NB, Ont, Que)

Trichuris spp. (Ont)

ACANTHOCEPHALA

Macracanthorhynchus hirudinaceus (Ont)

\section{CHELICERATA}

ACARI

Demodex phylloides (Ont)

Dermacentor andersoni (BC)

Sarcoptes scabiei (NB, Ont, Que)

UNIRAMIA

SIPHUNCULATA

Haematopinus suis (BC, Ont, Que)

Haematopinus spp. (Ont)

\section{ORDER CARNIVORA}

\section{FAMILY CANIDAE}

Canis familiaris Domestic Dog

PROTOZOA

Eimeria canis (Ont, Que)

Endolimax nana (Sask)

Entamoeba blattae (Que)

E. coli (Que, Sask)

E. histolytica (Que, Sask)

Giardia canis (Que, Sask)

Giardia spp. (Alta, NS, Ont)

Isospora bigemina (Que, Sask)

I. canis (Sask)

I. felis (Ont, Que)

I. ohioensis (Sask)

I. rivolta (Ont, Que)

I. wallacei (Sask)

Isospora spp. (Alta, NB, NWT, Que, Sask)

Toxoplasma gondii (Ont, Que)

Toxoplasma spp. (Ont)

Trichomonas spp. (Ont)

\section{TREMATODA}

Alaria arisaemoides (Sask)

A. canis (Ont)

Alaria spp. (Alta, NWT, Ont, Que, Sask)

Cryptocotyle lingua (Nfld, Que)

Metorchis conjunctus (Alta, Man, NWT,

Ont, Que, Sask)

Metorchis spp. (Sask)

Nanophyetus salmincola $(\mathrm{BC})$

Paragonimus kellicotti(Ont)

Paragonimus spp. (Ont)

Parametorchis complexus (Man)

Unidentified Trematoda (Man, NWT, Que) 


\section{CESTOIDEA}

Diphyllobothrium latum (Alta, Nfld, NWT,

Que, Sask)

Diphyllobothrium spp. (Alta, Man, Nfid,

NWT, Ont, Que, Sask)

Dipylidium caninum (NB, Nfld, NWT, Ont)

Dipylidium spp. (Ont, Sask)

Echinococcus granulosus (Ont, Que)

Mesocestoides spp. (Ont)

Taenia crassiceps (Ont)

T. elliptica (Que)

T. hydatigena (Nfld, Que)

T. pisiformis (Alta, Nfld, NWT, Ont, Que)

T. serialis (Alta, NWT)

Taenia spp. (BC, Man, NWT, Ont, Que,

Sask)

Taenia/Echinococcus spp. (egg) (Alta, NWT,

Sask)

Unidentified Cestoidea (Man, Que)

\section{NEMATODA}

Ancylostomatidae gen. sp. (Alta, NB, NWT,

Que, Sask)

Ancylostomum caninum (Alta, Ont, Que)

Ancylostomum spp. (Ont, Sask)

Ascaris spp. (Que)

Capillaria aerophila (Que)

Capillaria spp. (Ont)

Crenosoma vulpis (Nfld)

Dioctophyma renale (Man, NWT, Ont, Que)

Dipetalonema spp. (Ont)

Dirofilaria immitis (Alta, Ont, Sask)

Dirofilaria spp. (Ont)

Dracunculus insignis (Que)

Filaroides milksi $(\mathrm{BC})$

Filaroides spp. (Ont)

Oslerus osleri (Que)

Pelodera strongyloides (Ont)

Physaloptera rara (Que)

Spirocerca spp. (Ont)

Strongyloides stercoralis (Que)

Strongyloides spp. (Ont)

Toxascaris leonina (Alta, Man, NB, Nfld, NS,

NWT, Ont, Que, Sask)

Toxascaris spp. (Ont, Sask)

Toxocara canis (Alta, Man, NB, Nfld, NS,

NWT, Ont, Que, Sask)

Toxocara spp. (Ont, Sask)

Trichuris vulpis (Nfld, Ont, Que)

Trichuris spp. (Alta, NWT, Ont, Sask)

Trichuris spp. egg (NB, Que, Sask)

Uncinaria stenocephala (Alta, BC, Man, Nfld,

NWT, Ont, Que)

Uncinaria spp. (Ont, Sask)

Unidentified Nematoda (Sask)

Unidentified Rhabditoidea (Ont)

\section{ACANTHOCEPHALA}

Corynosoma semerme (NWT)

\section{CHELICERATA}

ACARI

Cheyletiella spp. (Ont, Sask)

Demodex spp. (Ont)

Dermacentor albipictus (Sask)

D. andersoni $(\mathrm{BC})$

D. variabilis (Man, Ont)

Ixodes cookei (BC, NB, Ont, Que)

I. ricinus (BC)

I. ricinus var. scapularis $(\mathrm{BC})$

Ixodes spp. (Ont)

Notoedres spp. (Ont)

Otobius spp. (Ont)

Otodectes cynotis (Que)

O. cynotis var. canis $(\mathrm{BC})$

Pneumonyssus spp. (Ont)

Rhipicephalus sanguineus (BC, NS, Ont)

Rhipicephalus spp. (Ont)

Sarcoptes scabiei (Ont)

Trombiculidae gen. sp. (Ont)

UNIRAMIA

DIPTERA

Cuterebra spp. (Ont)

MALLOPHAGA

Trichodectes canis $(\mathrm{BC})$

Trichodectes spp. (Ont)

SIPHONAPTERA

Ctenocephalides canis (Alta, BC, NB, Ont)

C. felis (BC, NS)

C.f. felis (BC, Man, NB, Nfld, NS, Ont, Que,

Sask)

Ctenocephalides felis felis (BC, Man, NB,

Nfld, NS, Ont, Que, Sask)

Tamiophila grandis (NB)

\section{SIPHUNCULATA}

Linognathus setosus (BC, Man, Ont, Que)

Linognathus spp. (Ont)

\section{FAMILY FELIDAE}

Felis catus Domestic Cat

PROTOZOA

Isospora felis (Ont)

I. rivolta (Ont)

Isospora spp. (NB)

Toxoplasma gondii (Ont, Sask)

Toxoplasma spp. (Ont, Que)

\section{TREMATODA}

Metorchis conjunctus (Ont, Que)

Paragonimus kellicotti(Ont)

Parametorchis complexus (Alta)

Plagiorchis massino (Nfld) 


\section{CESTOIDEA}

Diphyllobothrium dendriticum (Nfld)

Diphyllobothrium spp. (Ont)

Dipylidium caninum (Nfld, Ont)

Dipylidium spp. (Ont)

Echinococcus multilocularis (Sask)

Taenia taeniaeformis (Nfld, Que)

Taenia spp. (Ont)

\section{NEMATODA}

Aelurostrongylus spp. (Ont)

Ancylostomum spp. (Ont)

Capillaria aerophila (Ont)

Capillaria hepatica (NB)

Capillaria spp. (Ont)

Dirofilaria immitis (Ont)

Ollulanus tricuspis (Alta, Ont, Que)

Rhabditoidea gen. sp. (Ont)

Strongyloides spp. (Ont)

Toxascaris leonina $(\mathrm{NB}, \mathrm{Nfld})$

Toxascaris spp. (Ont)

Toxascaris cati (NB, Nfld, NS, Que)

Toxocara spp. (Ont)

\section{CHELICERATA}

\section{ACARI}

Dermacentor andersoni (BC)

Dermacentor spp. (Ont)

Haemophysalis leporis-palustris (BC)

Ixodes cookei (NB, Ont, Que)

Ixodes spp. (Ont)

Otobius lagophilus (Alta)

O. megnini (BC)

Otodectes cynotis (BC, NB, Que)

O. cynotis var. felis (BC)

Sarcoptes scabiei (unspecified)

\section{UNIRAMIA}

DIPTERA

Calliphoridae gen. sp. (Ont)

Cuterebra spp. (Ont)

\section{MALLOPHAGA}

Felicola subrostratus (BC, Que)

\section{SIPHONAPTERA}

Ctenocephalides canis (Alta, BC)

C. felis (BC, NS)

C. f. felis (Alta, BC, Man, Nfld, NB, NS, Ont,

Que, Sask)

Ctenocephalides spp. (Ont)

Monopsyllus vison (Sask)

\section{ORDER PERISSODACTYLA}

\section{FAMILY EQUIDAE}

\section{Equus caballus Domestic Horse

Eimeria crandallis (Ont)

E. granulosa (Ont)

E. intricata (Ont)

E. leuckarti(Ont)

Klossiella equi (NB)

Sarcocystis spp. (Ont)

Toxoplasma gondii(Ont)

Toxoplasma spp. (Ont, Sask)

Trichomonas spp. (Ont)

Trypanosoma equiperdum (Alta)

\section{CESTOIDEA}

Anoplocephala magna (Ont, Que)

A. perfoliata (Nfld, Ont)

Anoplocephala spp. (Ont)

Paranoplocephala mamillana (BC, Que, Sask)

\section{NEMATODA}

Capillaria hepatica (Alta)

Citellinema bifurcatum (Sask)

Cyathostoma spp. (Nfld)

Cyathostomum catinatum (Alta, Nfld, Sask)

C. coronatum (Sask)

C. labiatum (Alta, Sask)

C. labratum (Alta, Sask)

Cylicocyclus elongatus (Nfld, Sask)

C. insigne (Alta)

C. nassatus (Alta, Nfld, Sask)

C. radiatus (Alta, Sask)

Cylicodontophorus bicornatus (Alta, Sask)

Cylicostephanus calicatus (Alta, Sask)

C. goldi (Alta, Sask)

C. poculatus (Alta, Nfld, Sask)

Dictyocaulus arnfieldi (Ont)

Dictyocaulus spp. (Ont)

Gyalocephalus capitatus (Alta, NB, Ont, Sask)

Gyalocephalus spp. (NB)

Habronema majus (Ont)

H. muscae (Ont)

Habronema spp. (Ont)

Nematoda gen. sp. (Nfld)

Nematodirus spathiger (Que)

Oesophagodontus robustus (Alta, Nfld, Ont,

Que, Sask)

Oesophagodontus spp. (NB)

Onchocerca cervicalis (BC, Que)

Onchocerca sp. (Alta, BC, Man, Sask)

Oxyuris equi (NB, Nfld, Ont, Que)

Oxyuris spp. (Ont)

Parascaris equorum (NB, Nfld, Ont, Que)

Parascaris spp. (Ont)

Poteriostomum spp. (NB)

Probstmayria vivipara (NB, Que)

Setaria equina (Ont, Que)

Setaria spp. (Ont)

Strongyles gen. sp. (NB, Ont, Que)

Strongyloides spp. (Ont)

Strongylus edentatus (Alta, NB, Nfld, Ont,

Que, Sask) 
S. equinus (Alta, NB, Ont, Que, Sask)

S. vulgaris (Alta, NB, Nfld, Ont, Que, Sask)

Strongylus spp. (NB, Ont)

Thelazia lacrymalis (Ont, Que)

Thelazia spp. (Ont)

Triodontophorus brevicauda (Alta, Que,

Sask)

T. minor (Nfld)

T. serratus (Alta, Nfld, Ont, Que, Sask)

T. tenuicollis (Alta, Sask)

Triodontophorus spp. (NB, Ont)

Unidentified Nematoda (Nfld)

\section{CHELICERATA}

\section{ACARI}

Chorioptes spp. (Ont)

Dermacentor albipictus (Alta, BC, NB, NS,

Sask)

D. andersoni (Alta, BC)

Dermacentor spp. (Ont)

Psoroptes spp. (Ont)
UNIRAMIA

DIPTERA

Gasterophilus intestinalis (Alta, Ont)

G. nasalis (Alta, Ont)

Gasterophilus spp. (Man)

Hypoderma spp. (Ont)

\section{MALLOPHAGA}

Damalinia equi (BC)

Damalinia spp. (Ont)

\section{SIPHUNCULATA}

Haematopinus asini (BC, Que)

Haematopinus spp. (Ont) 


\section{References}

ALLEN, G.W. 1962. Acute atypical bovine pneumonia caused by Ascaris lumbricoides. Canadian Journal of Comparative Medicine and Veterinary Science 26: 241-243.

ALLEN, J.A., AND R.A. WARDLE. 1934. Fluke disease in northern Manitoba sledge dogs. Canadian Journal of Research 10: 404-408.

ALLEN, J.R., AND J.H.L. MILLS. 1971. Alaria arisaemoides in Saskatchewan dogs. Canadian Veterinary Journal 12: 24-28.

AMIN, O.M. 1982. Acanthocephala. pp. 933-940. In. Synopsis and Classification of Living Organisms. McGraw-Hill Book Co., New York.

ANDERSON, R.C., A.G. CHABAUD, AND S. WILLMOTT, eds. 1974-1983. CIH Keys to the Nematode Parasites of Vertebrates Nos. 1-10. Commonwealth Agricultural Bureaux, Farnham Royal, Bucks, England.

ANDERSON, R.C., AND U.R. STRELIVE. 1966. Experimental cerebrospinal nematodiasis (Pneumostrongylus tenuis) in sheep. Canadian Journal of Zoology 44: 889-894.

ANVIK, J.O., A.E. HAGUE, AND A. RAHAMAN. 1974. A method of estimating urban dog populations and its application to the assessment of canine fecal pollution and endoparasitism in Saskatchewan. Canadian Veterinary Journal 15: 219-223.

AUSTIN, R.J., AND K.H. DIES. 1981. Klossiella equi in the kidneys of a horse. Canadian Veterinary Journal 22: 159-161.

AXELSON, R.D. 1962. Metorchis conjunctus liver fluke infestation in a cat. Canadian Veterinary Journal 3: 359-360.

AYALEW, L., J.L. FRECHETTE, R. MALO, AND C. BEAUREGARD. 1973a. Gastrointestinal nematode populations in stabled ewes of Rimouski region. Canadian Journal of Comparative Medicine 37: 356-361.

1973b. Studies on the incidence of Dictyocaulus filaria in sheep of Rimouski region. Canadian Veterinary Journal 14: $301-304$

1974. Seasonal fluctuations and inhibited development of populations of Dictyocaulus filaria in ewes and lambs. Canadian Journal of Comparative Medicine 38:448-456.

AYALEW, L., AND H.C. GIBBS. 1973. Seasonal fluctations of nematode populations in breeding ewes and lambs. Canadian Journal of Comparative Medicine 37: 79-89

BARKER, I.K. 1970. Thelazia lacrymalis from the eyes of an Ontario horse. Canadian Veterinary Journal 11: 186-189.

BASIR, M.A. 1950. The morphology and development of the sheep nematode, Strongyloides papillosus (Wedl, 1856). Canadian Journal of Research 28: 173-196.
BATEMAN, D.G., J.D. BAIRD, J.O. SLOCOMBE, K.E LESLIE, R.A. CURTIS, AND P.I. MENZIES. 1986. Verminous pneumonia in adult dairy cows in southern Ontario due to Dictyocaulus viviparous. Canadian Veterinary Journal 27: 233-236.

BEAUREGARD, M., S.E. MAGWOOD, G.L. BANNISTER, A. ROBERTSON, P. BOULANGER, G.M. RUCKERBAUER, AND A.M. APPEL. 1965. A study of toxoplasma infection in chickens and cats on a family farm. Canadian Journal of Comparative Medicine and Veterinary Science 29: 286-291.

BEQUAERT, J.C. 1945. The ticks, or Ixodoidea, of the northeastern United States and eastern Canada. Entomologica Americana 25: 121-224.

BERNARD, M.A. 1970. Feline dirofilariasis. Canadian Veterinary Journal 11: 190-191.

1971. Dirofilariasis in Canada. Canadian Veterinary Journal 12: 139.

BLAKLEY, B.R. 1979. A livestock disease survey from auction mart inspection in Saskatchewan. Canadian Veterinary Journal 20: 358-360.

BLITZ, N.M., AND H.C. GIBBS. 1971. Morphological characterization of the stage of arrested development of Haemonchus contortus in sheep. Canadian Journal of Zoology 49: 991-995.

BOOTH, A.J., L STOGDALE, AND J.A. GRIGOR. 1984. Salmon poisoning in dogs on southern Vancouver Island. Canadian Veterinary Journal 25: 2-6.

BOURNS, T.K.R., L.R. MARIEN, AND D.H. PERCY. 1975. A search for heartworm in southern Ontario dogs. Canadian Veterinary Journal 16: 244.

BOUVRY, M., AND M.E. RAU. 1984. Paramphistomum spp. in dairy cattle in Quebec. Canadian Veterinary Journal 25: 353-356.

1986. Seasonal variations in egg passage of Fasciola hepatica in dairy cows in Quebec. Veterinary Parasitology 22: 267-273.

BROOKS, D.R., R.T. O'GRADY, AND D.R. GLEN. 1985. Phylogenetic analysis of the Digenea (Platyhelminthes: Cercomeria) with comments on their adaptive radiation. Canadian Journal of Zoology 63: 411-443.

BROWN, J.H. 1944a. The fleas (Siphonaptera) of Alberta, with a list of the known vectors of sylvatic plague. Annals of the Entomological Society of America 37: 207-213.

1944b. The spotted fever and other Albertan ticks. Canadian Journal of Research 22: 36-51.

BROWN, J.H., AND G.M. KOHLS. 1950. The ticks of Alberta with special reference to distribution. Canadian Journal of Research 28: 197-205.

BROWN, N.R. 1955. Additional host and distribution records of some eastern Canadian Siphonaptera. Canadian Entomologist 87: 362-365. 
1968. Notes on the Siphonaptera of New Brunswick. Canadian Entomologist 100: 486-498.

BUCKNER, C.H. 1964. Fleas (Siphonaptera) of Manitoba mammals. Canadian Entomologist 96: 850-856.

BURT, A.D., J.S. GAMMIE, AND S.E. ELLIS, 1978. A case of tick paralysis in a dog. Canadian Veterinary Journal 19: 347.

CALDWELL, D. 1984. Ollulanus tricuspis in an Ontario cattery. Canadian Veterinary Journal 25: 314.

CAMERON, A.E., AND J.S. FULTON. 1927. A local outbreak of the winter or moose tick, Dermacentor albipictus, Pack. ( Ixodoidea) in Saskatchewan. Bulletin of Entomological Research 17: 249-257.

CAMERON, T.W.M. 1938. Investigations on trichinosis in Canada. I. A preliminary survey of the incidence of Trichinella spiralis in hogs in eastern Canada. Canadian Journal of Research 16: 89-92.

1939. Investigations on trichinosis in Canada. II. A further survey of the incidence of Trichinella spiralis in hogs in eastern Canada. Canadian Journal of Research 17: 151-153.

1940a. Investigations on trichinosis in Canada. III. On the incidence of trichinosis in garbage-fed hogs. Canadian Journal of Research 18: 83-85.

1940b. Ollulanus tricuspis in the cat in Canada. Canadian Journal of Comparative Medicine 4: 296-297.

CAMERON, T.W.M., I.W. PARNELL, AND L.L. LYSTER. 1940. The helminth parasites of sledge-dogs in Northern Canada and Newfoundland. Canadian Journal of Research 18: 325-332.

CAMPBELL, D.J., D.L. DIAMOND, AND A.A. KINGSCOTE. 1960. Nematodiriasis in calves. Canadian Veterinary Journal 1: 119-122.

CAMPBELL, D.J., AND G.D. WETHERILL. 1957. Parasitic bronchitis in adult cattle in Ontario - a case report. Journal of the American Veterinary Medical Association 131: 273-275.

CHOQUETTE, L.P.E. 1945. Observation on the incidence of the common stomach worm, Haemonchus contortus, in young cattie in a Montreal abattoir. Canadian Journal of Comparative Medicine 9: 330-331.

1956. Observations on experimental infection of dogs with Echinococcus. Canadian Journal of Zoology 34: 190-192.

CHOQUETTE, L.P.E., AND L. de G. GELINAS. 1950. The incidence of intestinal nematodes and protozoa in dogs in the Montreal district. Canadian Journal of Comparative Medicine 14: 33-38.

CLARK, E.G., H.G.G. TOWNSEND, AND N.T. McKENZIE. 1981. Equine protozoal myeloencephalitis: A report of two cases from western Canada. Canadian Veterinary Journal 22: 140-144.

CLERMONT, R. AND R. RUPPANNER. 1975. Aspects epizootiologiques et economiques de l'hypodermose (Hypoderma sp.) bovine dans le comte de megantic. Canadian Veterinary Journal 16: 170-173.
COMFORT, C.M., AND R.D. AXELSON. 1962. Two reports of unusual parasites diagnosed in dogs. Canadian Veterinary Journal 3: 22-24.

CONKLIN, R.L., AND A.D. BAKER. 1930. Presence of the lancet fluke, Dicrocoelium dendriticum (Rudolphi, 1819), in Canada. Journal of Parasitology 17: 18-19.

COOLEY, R.A., AND G.M. KOHLS. 1945. The genus Ixodes in North America. U.S. Public Health Service, National Institute of Health Bulletin No. 184, 246 pp.

CRIBB, A.E., AND D. SPRACKLIN. 1986. Giardiasis in a home. Canadian Veterinary Journal 27: 169.

DAVENPORT, P.G., AND P.H.G. STOCKDALE. 1967. A case of hyostrongylosis in Ontario. Canadian Veterinary Journal 8: 288-290.

DEROTH, L., AND J.L. FRECHETTE. 1975. Dracunculus insignis (Leidy, 1858) chez un berger allemand. Canadian Veterinary Journal 16: 176-178.

DIES, K.H., AND J. PRITCHARD. 1985. Bovine Stephanofilarial dermatitis in Alberta. Canadian Veterinary Journal 26: 361-362.

DUKES, T.W., A. WEBSTER, AND A. BUNDZA. 1981. Mineralized onchocercal lesions resembling tuberculosis in cattle. Report of cases from meat inspection. Canadian Veterinary Journal 22: 12-14.

EMERSON, K.C. 1972. Checklist of the Mallophaga of North America (North of Mexico). Deseret Test Center. Dugway, Utah.

FALLIS, A.M. 1938. A study of the helminth parasites of lambs in Ontario. Transactions of the Royal Canadian Institute 22: 81-128.

1940. Studies on Oestrus ovis L. Canadian Journal of Research 18: 442-446.

FAUBERT, G.M., P. VIENS, AND P. MAGLUILO. 1985. Superiority of the ELISA technique over parasitological methods for detection of trichinellosis in slaughtered pigs in Canada. Canadian Journal of Comparative Medicine 49: 75-78.

FLETCH, A.L. 1969. Pneumothorax in a dog with Dirofilaria immitis infection. Canadian Veterinary Journal 10: 80-82.

FRANK, J.F. 1952. A study of the incidence of Trichinosis in swine in the maritime provinces. Canadian Journal of Comparative Medicine 16: 73-77.

FRECHETTE, J.L., AND H.C. GIBBS. 1971. Studies on the incidence of gastrointestinal helminths of cattle in Quebec. Canadian Veterinary Journal 12: 207-210.

FRECHETTE, J.L., M. BEAUREGARD, A.L. GIROUX, AND D. CLAIRMONT. 1973. Infection de jeunes bovins par Trichuris discolor. Canadian Veterinary Journal 14: 243-246.

FRECHETTE, J.L., M. MARCOUX, AND H. ST. PIERRE. 1976. Presence de Thelazia sp. au Quebec chez le cheval et la Vaache. Canadian Veterinary Journal 17: 114. 
FREEMAN, R.S. 1962. Studies on the biology of Taenia crassiceps (Zeder, 1800) Rudolphi, 1810 (Cestoda). Canadian Journal of Zoology 40: 969-990.

FREEMAN, R.S., A. ADORJAN, AND D.H. PIMLOTT. 1961. Cestodes of wolves, coyotes, and coyote-dog hybrids in Ontario. Canadian Journal of Zoology 39: 527-532.

FRIMETH, J.P., AND H.P. ARAI. 1983. Some potential mosquito vectors of the canine heartworm, Dirofilaria immitis, in the Calgary region of southern Alberta. Canadian Journal of Zoology 61: 1156-1158.

GAJADHAR, A.A., J.P. CARON, AND J.R. ALLEN. 1985. Cryptosporidiosis in two foals. Canadian Veterinary Journal 26: 132-134.

GAJADHAR, A.A., W.D.G. YATES, AND J.R. ALLEN. 1987. Association of Eosinophilic Myositis with an unusual species of Sarcocystis in a beef cow. Canadian Journal of Veterinary Research 51: 373-378.

GALLOWAY, T.D., AND J.A. HARRIES. 1982. A survey for cattle grubs (Diptera: Hypoderma spp.) in slaughtered cattle in Manitoba packing plants, 1980. Canadian Journal of Animal Science 62: 935-941.

GHADIRIAN, E., P. VIENS, H. STRYKOWSKI, AND F. DUBREUIL. 1976. Epidemiology of toxocariasis in the Montreal area. Canadian Journal of Public Health 67: 495-498.

GIBBS, H.C. 1964. Observations on an outbreak of clinical parasitism in ewes during the winter months. Canadian Veterinary Journal 5: 8-11.

GILLICK, A. 1972. Paragonimiasis in a dog. Canadian Veterinary Journal 13: 175-179.

GRAESSER, F.E. 1957. Lungworm disease of cattle in Alberta. Canadian Journal of Comparative Medicine 21: 355-358.

GREENWAY, J.A., AND P.H.G. STOCKDALE. 1970. A case tentatively diagnosed as Filaroides milksi in a dog. Canadian Veterinary Journal 11: 203-204.

GREGSON, J.D. 1935. The production of artificial conditions conducive to winter feeding of ticks. Proceedings of the B.C. Entomological Society (1935): 6-7.

1937. Notes on some phenomenal feeding in ticks. Proceedings of the B.C. Entomological Society (1937): 8-11.

1947. Feeding periods prerequisite to the mating of Dermacentor andersoni. Proceedings of the B.C. Entomological Society (1946) 43: 3-6.

1952. Further studies on tick paralysis. Proceedings of the B.C. Entomological Society 48: 54-58.

1953. Records of the tick Otobius megnini (Duges) from British Columbia (Acarina: Argasidae). Proceedings of the B.C. Entomological Society 49: 30.

1958a. Host susceptibility to paralysis by the tick Dermacentor andersoni Stiles (Acarina: Ixodidae). Canadian Entomologist 90: 421-424. 1958b. Recent cattle grub life-history studies at Kamloops, British Columbia, and Lethbridge, Alberta. 10th International Congress of Entomology 3, (1958): 725-734.

1958c. Tick paralysis in cattle in British Columbia in 1957. Proceedings of the B.C. Entomological Society 55: 6-7.

GRIFFITHS, H.J. 1937. Some observations on the overwintering of certain helminth parasites of sheep in Canada. Canadian Journal of Research 15: 156-162.

1939. Distribution of Fasciola hepatica Linn. and its potential vectors in Canada. Scientific Agriculture 20: 166-168.

GUPTA, R.P., AND H.C. GIBBS. 1969. Studies on the incidence of lungworm (Dictyocaulus viviparous, Bloch, 1782) in Quebec cattle. Canadian Veterinary Journal 10: 279-285.

GUPTA, R.P., AND H.C. GIBBS. 1975. Infection patterns of Dictyocaulus viviparous in calves. Canadian Veterinary Journal 16: 102-108.

HADWEN, S. 1913. On "Tick Paralysis" in sheep and man following bites of Dermacentor venustus, with notes on the biology of the tick. Parasitology 6: 283-297.

HALL, R.R., AND McKIEL, J.A. 1961. Occurrences of the American dog tick, Dermacentor variabilis (Say) in western Nova Scotia. Canadian Entomologist 93: 891-893.

HARVEY, R.V. 1907. British Columbia fleas. Bulletin of the Entomological Society of British Columbia No. 7.

HEARLE, E. 1933. Notes on a serious outbreak of tick paralysis in cattle. Proceedings of the B.C. Entomological Society (1933): 11-16.

1938. The ticks of British Columbia. Scientific Agriculture 18: 341-354.

HEWITT, C.G. 1915. A contribution to a knowledge of Canadian ticks. Transactions of the Royal Society of Canada 9(series 3): 225-239.

HOLLAND, G.P. 1940. Notes on the ecology of Dermacentor andersoni in southern Alberta. Proceedings of the B.C. Entomological Society 36: 11-12.

1984. The fleas of Canada, Alaska and Greenland (Siphonaptera). Memoirs of the Entomological Society of Canada No. 130.631 pp.

HOPKINS, G.H.E. 1960. Notes on some Mallophaga from mammals. Bulletin of the British Museum of Natural History (Entomology) 10: 77-98.

HORTON, G.M.J., AND P.H.G. STOCKDALE. 1981. Oocyst discharge, rumen metabolism and performance of early weaned lambs with naturally occurring coccidiosis fed monensin. Canadian Veterinary Journal 22: $175-178$.

HULLAND, T.J. 1956. Toxoplasmosis in Canada. Journal of the American Veterinary Medical Association 128: 74-79. 
JARVIS, T.D. 1910. The Acarina, with a host index to the species found in Ontario. 40th Annual Report of the Entomological Society of Ontario 36: 82-109.

JOHNSTON, E.F., AND L.W. MacPHERSON. 1956. An outbreak of acute parastic gastroentritis in a herd of cattle in the Ottawa Valley. Canadian Journal of Comparative Medicine 20: 203-205.

JUDD, W.W. 1953. Mammal host records of Acarina and Insecta from the vicinity of London, Ontario. Journal of Mammalogy 34: 137-139.

1954. Some records of ectoparasitic Acarina and Insecta from mammals in Ontario. Journal of Parasitology 40: $483-484$.

JULIAN, R.J. 1971. The isolation of trypanosomes from cattle in Ontario. Canadian Journal of Comparative Medicine 35: 192-194.

KENNEDY, M.J. 1983. A revision of North American species of the Genus Parametorchis Skrjabin, 1913 (Trematoda: Opisthorchiidae). Proceedings of the Helminthological Society of Washington 50: 312-317.

KENNEDY, M.J., AND R.A. KRALKA. 1986. A survey of ectoparasites on cattle in central Alberta, November 1984 - July 1985. Canadian Veterinary Journal 27: $459-460$.

KENNEDY, M.J., AND R.A. KRALKA. 1987. A survey of Eimeria spp. in cattle in central Alberta. Canadian Veterinary Journal 28: 124-125.

KENNEDY, M.J., R.A. KRALKA, AND M. SCHOONDERWOERD. 1984. First report of Ollulanus tricuspis (Nematoda) from western Canada. Journal of Parasitology 70: 319-320.

KENNEDY, M.J., AND D. MORAIKO. 1987. The eyeworm, Thelazia skrjabini in cattle in Canada. Canadian Veterinary Journal 28: 254-255.

KHAN, M.A. 1981. An intradermal test to detect latent warble (Hypoderma spp.) infection in cattle. Canadian Veterinary Journal 22: 36-41.

KHAN, M.A., AND G.B. SCHAALJE. 1985. Chlorpyrifos for control of the short-nosed cattle louse, Haematopinus eurysternus (Nitzsch) (Anoplura, Haematopinidae) during winter. Canadian Journal of Comparative Medicine 49: 361-365.

KRANTZ, G.W. 1978. A manual of Acarology. 2nd ed. Oregan University Book Store Inc. Corvallis. 509 pp.

LaRUE, G.R., AND A.M. FALLIS. 1934. Alaris canis n. sp. (Alaridae), a trematode parasite of the dog in northern Ontario. Journal of Parasitology 20: 335.

LAVIGNE, C., AND H.J. SMITH. 1983. Treatment of sarcoptic manage in Canadian cattle with ivermectin. Canadian Veterinary Journal 24: 389-391.

LEES, M.J., N. KLEIDER, AND T.J. TUDDENHAM. 1983. Cutaneous onchocerciasis in the horse: five cases in southwestern British Columbia. Canadian Veterinary Journal 24:3-5.
LEVINE, N.D. (Chairman). 1980. A newly revised classification of the Protozoa. By The Committee on Systematics and Evolution of the Society of Protozoologists. Journal of Protozoology 27:37-58.

LEWIS, JR., P.D. 1974. Presence of Dicrocoelium dendriticum (Rudolphi, 1819) (Trematoda:

Dicrocoeliidae) in western Canada. Canadian Journal of Zoology 52: 662-663.

LITTLE, P.B. 1972. Cerebrospinal nematodiasis of Equidae. Journal of the American Veterinary Medical Association 160: 1407-1413.

LORD, L.H., AND J.K. McGREGOR. 1960. A case of Strongyloides dermatitis in Canada. Canadian Veterinary Journal 1: 111-114.

LYAL, C.H.C. 1987. Co-evolution of trichodectid lice (Insecta: Phthiraptera) and their mammalian hosts. Journal of Natural History 21: 1-28.

MACNAY, C.G. 1952. Summary of the more important insect infestations and occurrences in Canada in 1951. 82nd Annual Report of the Entomological Society of Ontario (1951). pp. 91-115.

1957. Summary of important insect infestations, occurrences and damage in Canada in 1957. Annual Report of the Entomological Society of Ontario (1957) 88: 63-78.

MADDY, K.T. 1955. Stephanofilarial dermatitis in cattle. The North American Veterinarian 36: 275-280.

MAHRT, J.J. 1969. Prevalence of coccidia in domestic sheep in central Alberta. Canadian Veterinary Journal 10: 176-178.

MALLOY, W.F., AND J.A. EMBIL. 1973. Prevalence of Toxocara sp. in dogs and cats in Halifax. Canadian Journal of Public Health 64: 84.

1978. Prevalence of Toxocara spp. and parasites in dogs and cats in Halifax, Nova Scotia. Canadian Journal of Comparative Medicine 42: 29-31.

MANN, E.D., L.H SEKLA, AND G. EIBISCH. 1987. Cryptosporidium antibodies in Manitoba cattle: A pilot study using an indirect fluorescent antibody procedure. Canadian Veterinary Journal 28: 126-128.

MANN, E.D., L.H. SEKLA, G.P.S. NAYAR, AND C. KOSCHIK. 1986. Infections with Cryptosporidium spp. in humans and cattle in Manitoba. Canadian Journal of Veterinary Research 50: 174-178.

MARCOUX, M., J.L. FRECHETTE, AND M. MORIN. 1977. Infection par Onchocerca cervicalis au Quebec: signes cliniques et methode de diagnostic. Canadian Veterinary Journal 18: 108-110.

MARGOLIS, L., AND J.R. ARTHUR. 1979. Synopsis of the Parasites of Fishes of Canada. Bulletin of the Fisheries Research Board of Canada No. 199. 269 pp.

MARTIN, L.J., H.C. GIBBS, AND J.W. PULLIN. 1974. Gastrointestinal parasites of swine in Quebec. I. An incidence survey. Canadian Veterinary Journal 15: 72-76. 
MARTIN, M., AND T. LETARTE. 1977. Prevalence of toxoplasmosis in Quebec farm dogs. The Veterinary Record 101: 79-80.

MARTINEAU, G.P., J. VAILLANCOURT, AND J.L. FRECHETTE. 1984. Control of Sarcoptes scabiei infestation with ivermectin in large intensive breeding piggery. Canadian Veterinary Journal 25: 235-238.

McCRAW, B.M., E.W. EATON, T.H. OGILVIE, T.E. BLACK WELL, AND D.G. BUTLER 1981.

Transmission and treatment of Muellerius capillaris in goats. Canadian Veterinary Journal 22: 205.

McCRAW, B.M., AND J.P. LAUTENSLAGER. 1971. Pneumonia in calves associated with migrating Ascaris suum larvae. Canadian Veterinary Journal 12: 87-90.

McCRAW, B.M., AND P.I. MENZIES. 1986. Treatment of goats infected with lungworm, Muellerius capillaris. Canadian Veterinary Journal 27: 287-290.

McGREGOR, J.K., AND A.A. KINGSCOTE. 1957. A survey of gastro-intestinal helminths of cattle in Ontario. Canadian Journal of Comparative Medicine 21: 370-373.

McLEOD, J.A. 1967. Dioctophyma renale infections in Manitoba. Canadian Journal of Zoology 45: 505-508.

MEADS, E.B. 1976. Dalmeny disease - another outbreak - probably Sarcocystis. Canadian Veterinary Journal 17: 271.

MILLS, J.H.L., AND R.S. HIRTH. 1968. Lesions caused by the hepatic tramatode Metorchis conjunctus, Cobbold, 1860. Journal of Small Animal Practics 9: 1-6.

MONGEAU, N. 1961. Hepatic distomatosis and infectious canine hepatitis in northern Manitoba. Canadian Veterinary Journal 2: 33-38.

MOOLENBEEK, W.J., AND G.A. SURGEONER. 1980. Southern Ontario survey of eyeworms, Thelazia gulosa and Thelazia lacrymalis in cattle and larvae of Thelazia spp. in the face fly, Musca autumnalis. Canadian Veterinary Journal 21: 50-52.

MORIN, M., Y. ROBINSON, AND D. TURGEON. 1980. Intestinal coccidiosis in baby pig diarrhea.

Canadian Veterinary Journal 21:65.

MORIN, M., D. TURGEON, J. JOLETTE, Y. ROBINSON, J.B. PHANEUF, R. SAUVAGEAU, M. BEAUREGARD, E. TEUSCHER, R. HIGGINS, AND S. LARIVIERE. 1983. Neonatal diarrhea of pigs in Quebec: infectious causes of significant outbreaks. Canadian Journal of Comparative Medicine 47: 11-17.

MOTEANE, M., D.M. MIDDLETON, AND L.R. POLLEY. 1979. A survey of disease conditions in adult and feeder sheep in Saskatchewan. Canadian Veterinary Journal 20: 2-7.

MOYNIHAN, I.W., AND I.W. MUSFELDT. 1949. A study of the incidence of trichinosis in swine in British Columbia. Canadian Journal of Comparative Medicine 13: 224-227.
MURRAY, V.I.E. 1967. Studies on Hypoderma lineatum de Villiers and Hypoderma bovis deGeer (Diptera: Hypodermatidae) in Manitoba. Canadian Veterinary Journal 8: 17-22.

NATION, P.N., AND J.R. ALLEN. 1976. Antibodies of Toxoplasma gondii in Saskatchewan cats, sheep and cattle. Canadian Veterinary Journal 17: 308-310.

NATION, P.N., AND K.H. DIES. 1978. Capillaria hepatica in a horse. Canadian Veterinary Journal 19: 315-316.

NELSON, W.A. 1952. A note on the presence of first-stage larvae of Gasterophilus intestinalis (Degeer) (Diptera: Gasterophilidae) in the mouth of the horse. Canadian Entomologist 84: 356.

NELSON, W.A., AND J. WEINTRAUB. 1972. Hypoderma lineatum (DeVill) (Diptera: Oestridae): Invasion of the bovine skin by newly hatched larvae. Journal of Parasitology 58: 614-624.

NIELSEN, S.W. 1955. Canine paragonimiasis. North American Veterinarian 36: 657-662.

NIILO, L. 1968. Bovine hemorrhagic filariasis in cattle imported into Canada. Canadian Veterinary Journal 9: 132-137.

NIMMO, J.S. 1979. Six cases of verminous pneumonia (Muellerius sp.) in goats. Canadian Veterinary Journal 20: 49-51.

NOWAK, R.M., AND J.L. PARADISO. 1983. Walker's Mammals of the World. 4th Edition, Vols. I and II. The Johns Hopkins University Press. Baltimore. 1362 pp. + XXV.

O'DONOGHUE, J.G. 1958. Clinical trials with Cyanacethydrazide for the treatment of lungworms in cattle and sheep. Canadian Journal of Comparative Medicine 22: 237-239.

OLSON, M.E. 1985. Coccidiosis caused by Isospera ohioensis-like organisms in three dogs. Canadian Veterinary Journal 26: 112-114.

OSLER, W. 1877. Verminous bronchitis in dogs. Veterinarian London 50: 387-397.

OSLER, W., AND A.W. CLEMENT. 1883. An investigation into the parasites in the pork supply of Montreal. 14 pp. Montreal Gazette Printing Co. 1883.

PALLISTER, E., AND P.J.G. PLUMMER. 1941. Multicystic hydatid in the liver of a hog. Canadian Journal of Comparative Medicine 5: 291-292.

PECHERE, J.C., R. LETARTE, Y. BRINDLE, R. HIGGINS, A. TURCOTTE, M. MARTIN. 1977. La toxoplasmose dans un milieu rural Quebecois. Canadian Journal of Public Health 68: 425-429.

POLLEY, L. 1984. Onchocerca in horses from western Canada and the northwestern United States: An abattoir survey of the prevalence of infection. Canadian Veterinary Journal 25: 128-129.

POLLEY, L., M.G. BICKIS. 1987. Gastrointestional nematode parasites in Saskatchewan cattle. Egg count 
distribution in beef animals. Canadian Journal of Veterinary Research 51: 465-469.

POLLEY, L.R., P.E. MOSTERT. 1980. Ascaris suum in Saskatchewan pigs: an abattoir survey of prevalence and intensity of infection. Canadian Veterinary Journal 21: 307-309.

RANSOM, B.H., AND S. HADWEN. 1918. Horse strongyles in Canada. Journal of the American Veterinary Medical Association 6: 202-214.

REID, S., AND D. MADILL. 1987. Trichuris species in cattle. Canadian Veterinary Journal 28:313.

RENDANO, V.T. Jr. 1974. Paragonimiasis in the cat: a review of five cases. Journal of Small Animal Practise 15: 637-644.

RICH, G.B. 1952. A review of studies on the systemic control of livestock insect parasites. Proceedings of the B.C. Entomological Society 48: 58-59.

1957. The ear tick, Otobius megnini (Duges) (Acarina: Argasidae), and its record in British Columbia. Canadian Journal of Comparative Medicine 21: 415-418.

ROBINSON, Y., AND M. MORIN. 1982. Porcine neonatal coccidiosis in Quebec. Canadian Veterinary Journal 23: 212-216.

SANFORD, S.E. 1987. Enteric cryptosporidial infection in pigs: 184 cases (1981 - 1985). Journal of the American Veterinary Medical Association 190: 695-698.

SANFORD, S.E., AND G.K.A. JOSEPHSON. 1981. Porcine neonatal coccidioses. Canadian Veterinary Journal 22: 282-285.

SANFORD, S.E., and G.K.A. JOSEPHSON. 1982. Bovine cryptosporidiosis: Clinical and pathological findings in forty-two scouring neonatal calves. Canadian Veterinary Journal 23: 343-347.

SAUNDERS, L.G. 1949. A survey of helminth and protozoan incidence in man and dogs at Fort Chipewyan, Alberta. Journal of Parasitology 35: 31-34.

SAUVAGEAU, R., AND J.L. FRECHETTE. 1980. Ascaridiose hepatique chez un agneau. Canadian Veterinary Journal 21: 66.

SCHMIDT, G.D. 1986. CRC Handbook of Tapeworm Identification. $688 \mathrm{pp}$.

SCHOLTEN, T.H., K. RONALD, and D.M. MCLEAN. 1962. Parasite fauna of the Manitoulin Island Region. Canadian Journal of Zoology 40: 605-606.

SEAH, S.K.K., G. HUCAL, AND C. LAW. 1975. Dogs and intestinal parasites: a public health problem. Canadian Medical Association Journal 112: 1191-1 194.

SLOCOMBE, J.O.D. 1970. Ostertagia trifurcata (Nematoda: Trichostrongylidae) in cattle in Canada. Canadian Journal of Zoology 48: 1143-1144.

1973a. Parasitisms in domesticated animals in Ontario. I. Ontario Veterinary College Records 1965-1970. Canadian Veterinary Journal 14: 36-42. 1973b. Gastrointestinal parasites in cattle in Ontario. Canadian Veterinary Journal 14: 91-95.

1973c. Morphological types in Haemonchus contortus (Nematoda) populations in Ontario sheep. Canadian Journal of Zoology 51: 1161-1163.

1974a. Abomasal nematodes in cattle in Ontario. Canadian Journal of Comparative Medicine 38: 18-21.

1974b. Overwintering of bovine gastrointestinal nematodes in southwestern Ontario. Canadian Journal of Comparative Medicine 38: 90-93.

1979. Prevalence and treatment of tapeworms in horses. Canadian Veterinary Journal 20: 136-140.

SLOCOMBE, J.O.D., AND J.F. COTE. 1984. Effectiveness of ivermectin paste for removal of nematodes in horses. Canadian Veterinary Journal 25: 386-388.

SLOCOMBE, J.O.D., AND B.M. McCRAW. 1973. Gastrointestinal nematodes in horses in Ontario. Canadian Veterinary Journal 14: 101-105.

SLOCOMBE, J.O.D., J. VALENZUELA, AND M.C. LAKE. 1987. Epidemiology of strongyles in ponies in Ontario. Canadian Journal of Veterinary Research 51: 470-474.

SMITH, F.R., AND W. THRELFALL. 1973. Helminths of some mammals from Newfoundland. American Midland Naturalist 90: 215-218.

SMITH, H.J. 1961. Bovine demodicidosis. I. Incidence in Ontario. Canadian Journal of Comparative Medicine 25: 165-169.

1970. On the development of gastrointestinal parasitisms in bovine yearlings. Canadian Journal of Comparative Medicine 34: 303-308.

1972. Anthelmintic activity of tetramisole against lungworms, roundworms, nodular worms, threadworms, and whipworms in a natural mixed infection of swine. Canadian Veterinary Journal 13: 40-44.

1973. On the immunological response of young calves to light Nematodirus helvetianus infection. Canadian Journal of Comparative Medicine 37: 320-322.

1974. Inhibited development of Ostertagia ostertagi, Cooperia onchophora, and Nematodirus helvetianus in parasite-free calves. American Journal of Veterinary Research 35: 935-938.

1975a. An evaluation of low temperature sterilization of Trichinae infected pork. Canadian Journal of Comparative Medicine 39: 362-363.

1975b. Trichinae in tail musculature of swine. Canadian Journal of Comparative Medicine 39: 362-363.

1976a. Strongyle infections in ponies. I. Response to intermittent thiabendazole treatments. Canadian Journal of Comparative Medicine 40: 327-333.

1976b. Strongyle infections in ponies. II. Reinfection of treated animals. Canadian Journal of Comparative Medicine 40: 334-340. 
1979a. Transmission of Oesophagostomum species in swine on pasture in the Maritime provinces. Canadian Veterinary Journal 20: 184-185.

1979b. Probstmayria vivipara pinworms in ponies. Canadian Journal of Comparative Medicine 43: 341-342.

1986. Transmission of Sarcoptes scabiei in swine by fomites. Canadian Veterinary Journal 27: 252-254.

SMITH, H.J., AND R. McG. ARCHIBALD. 1962. On the presence of Ostertagia species and Teladorsagia davtiani in sheep in Canada. Canadian Journal of Comparative Medicine and Veterinary Science 26: 273-274.

1965. The overwinter survival of ovine gastrointestinal parasites in the Maritime Provinces. Canadian Veterinary Journal 6: 257-267

1968. The effects of age and previous infection on the development of gastrointestinal parasitisms in cattle. Canadian Journal of Comparative Medicine 32: 511-517.

1969. On the survival of overwintering bovine gastrointestinal nematode larvae during the subsequent grazing season. Canadian Journal of Comparative Medicine 33: 44-47.

SMITH, H.J., AND A.B. HAWKES. 1978. Kidney worm infection in feral pigs in Canada with transmission to domestic swine. Canadian Veterinary Journal 19: 40-43.

SMITH, H.J., AND J.G. HINES. 1987. Nematodirus battus in Canadian sheep. Canadian Veterinary Journal 38: 256.

SMITH, H.J., AND E.D. KAY. 1987. Role of rats in the transmission of Trichinella spiralis spiralis to swine. Canadian Veterinary Journal 28: 604.

SMITH, H.J., AND J.P. PERREAULT. 1972. A type II ostertagiasis outbreak in cattle in New Brunswick. Canadian Veterinary Journal 13: 114-117.

SMITH, H.J., AND R.G. STEVENSON. 1970. A clincial outbreak of Trichuris discolor infection in stabled calves. Canadian Veterinary Journal 11: 102-104.

SOEHL, H. 1984a. Cysticercus ovis in Nova Scotia. Communication, Food Production and Inspection Branch, Agriculture Canada 2: 7-9.

1984b. An outbreak of Cysticercus ovis in Nova Scotia. Canadian Veterinary Journal 25: 424-425.

SPECKMAN, G., AND W.A. WEBSTER. 1975. Natural infection and treatment of a dog with Mesocestoides tapeworms. Canadian Veterinary Journal 16: 26-27.

SPENCER, G.J. 1936. A check list of the fleas of British Columbia with a note on fleas in relation to sawdust in homes. Proceedings of the B.C. Entomological Society 32: 11-17.

1938. Ectoparasites of birds and mammals of British Columbia. II. A preliminary list of the Pupipara, louse flies. Proceedings of the B.C. Entomological Society 34: $39-45$.
1939. Ectoparasites of birds and mammals in British Columbia. V. Parasites of domestic animals (Mammals). Proceedings of the B.C. Entomological Society 35: 19-23.

1940. Ectoparasites of birds and mammals in British Columbia. VI. A preliminary list of parasitic mites. Proceedings of the B.C. Entomological Society (1940): 14-18.

1960. The brown dog-tick, Rhipicephalus sanguineus (Latr.) in Vancouver. Proceedings of the B.C. Entomological Society 57: 64-65.

1966. Anoplura from British Columbia and some adjacent areas. Journal of the Entomological Society of British Columbia 63: 23-30.

STOCKDALE, P.H.G., G.K. ASHTON, M.A. HOWES, AND E. EWERT. 1973. Hyostrongylosis in Ontario. Canadian Veterinary Journal 14: 265-268.

STOCKDALE, P.H.G., AND W.N. HARRIES. 1979.

Treatment of feedlost cattle in Alberta for gastrointestinal nematodes. Canadian Veterinary Journal 20: 223-226.

STOCKDALE, P.H.G., AND J.P. LAUTENSLAGER. 1973. Unusual gastric nematodes of swine in Ontario. Canadian Veterinary Journal 14: 215-216.

STOGDALE, L. 1984. Testing for occult heartworm infection. Canadian Veterinary Journal 25: 171-174.

SWALES, W.E. 1933a. A Review of Canadian Helminthology. I. The present status of knowledge of the helminth parasites of domesticated and semidomesticated mammals and economically important birds in Canada, as determined from work published prior to 1933. Canadian Journal of Research 8: 468-477.

1933b. A review of Canadian Helminthology. II. Additions to part I, as determined from a study of parasitic helminths collected in Canada. Canadian Journal of Research 8: 478-482.

1939. Tests of Phenothiazine, a highly efficient anthelmintic. On a means of administration and the indicated uses for the control of parasitic diseases of sheep. Canadian Journal of Comparative Medicine 3: 188-194.

1940a. The helminth parasites and parasitic diseases of sheep in Canada. I. A survey and some preliminary studies on existing problems. Canadian Journal of Research 18: 29-48.

$1940 \mathrm{~b}$. The helminth parasites and parasitic diseases of sheep in Canada. II. Notes on the effect of winter upon the free-living stages of nematode parasites of sheep on the pastures in Eastern Canada. Canadian Journal of Comparative Medicine and Veterinary Science 4: 155-161.

SWALES, W.E., AND L. CHOQUETTE. 1940. Diagnostic et traitement des maladies parasitaires du mouton dans la province de Quebec. Canadian Journal of Comparative Medicine 4: 292-294. 
SWALES, W.E., P.E. SYLVESTRE, AND S.B.

WILLIAMS. 1942. Field trials of control measures for parasitic diseases of sheep. Canadian Journal of Research 20: 115-132.

SWEATMAN, G.K. 1958. Biology of Otodectes cynotis, the ear canker mite of carnivores. Canadian Journal of Zoology 36: 849-862.

SWEATMAN, G.K., AND P.J.G. PLUMMER. 1957. The biology and pathology of the tapeworm Taenia hydatigena in domestic and wild hosts. Canadian Journal of Zoology 35: 93-109.

TAILYOUR, J.M., AND J.R. STEELE. 1960. Trichinella infection in swine in the Vancouver area. Canadian Journal of Public Health 51: 309.

TESKEY, H.J. 1960. Survey of insects affecting livestock in southwestern Ontario. Canadian Entomologist 92: 531-544.

THOMPSON, G.B. 1934. Records of Siphunculata and Mallophaga from Canadian hosts. Canadian Entomologist 66: 279-281.

THRELFALL, W. 1969. Further records of helminths from Newfoundland mammals. Canadian Journal of Zoology 47: 197-201.

TIZARD, I.R., M. CARRINGTON, AND C.H. LAI. 1977. Toxoplamosis in goats in southern Ontario - A public health hazard? Canadian Journal of Comparative Medicine. 42: 177-183.

TIZARD, I.R., J. HARMESON, AND C.H. LAI. 1978. The prevalence of serum antibodies to Toxoplasma gondii in Ontario mammals. Canadian Journal of Comparative Medicine 42: 177-183.

TYRRELL, J.B. 1883. On the occurrence in Canada of two species of parasitic mites. Proceedings of the Canadian Institute 1: 332-342.

UNRUH, D.H.A., J.E. KING, R.D.P. EATON, AND J.R. ALLEN. 1973. Parasites of dogs from Indian settlements in northwestern Canada: A survey with Public Health Implications. Canadian Journal of Comparative Medicine 37: 25-32.

WALLIS, P.M., AND R.M., ZAMMUTO, AND J.M. BUCHANAN-MAPPIN. 1986. Cysts of Giardia spp. in mammals and surface waters in southwestern Alberta. Journal of Wildlife Diseases 22: 115-118.

WARDLE, R.A., AND E.L. McCOLL. 1937. The taxonomy of Diphyllobothrium latum (Linne, 1758) in Western Canada. Canadian Journal of Research 15: 163-175.

WATSON, E.A., AND S. HADWEN. 1912. Trypanosomes found in Canadian mammals. Parasitology 5: 21-26.

WATSON, T.G. 1981. Growth of Metorchis conjunctus (Cobbold, 1860) Looss, 1899 (Trematoda: Opisthorchiidae) in the bile ducts of various definitive hosts. Canadian Journal of Zoology 59: 2014-2019.
WEBSTER, G.A. 1958. On prenatal infection and the migration of Toxocara canis Werner, 1782, in dogs. Canadian Journal of Zoology 36: 435-440.

WEBSTER, W.A., and D.B. WILKINS. 1970. The recovery of Parafilaria bovicola Tubangui, 1934 from an imported Charolais bull. Canadian Veterinary Journal 11: 13-14.

WEBSTER, W.A., T.W. DUKES, AND A. BUNDZA. 1977. Onchocerciasis (Nematoda: Filariodea) in bovine leg joints. Canadian Journal of Zoology 55: 1067-1070.

WEBSTER, W.A., AND T.W. DUKES. 1979. Bovine and equine onchocerciasis in eastern North America with a discussion on cuticular morphology of Onchocerca sp. in cattle. Canadian Journal of Comparative Medicine 43: $330-332$.

WEBSTER, W.A., R.R. MacKAY. 1969. Two new host records for Nematodirus species. Canadian Journal of Zoology 47: 1411.

WEINTRAUB, J. 1952. A preliminary report on acaricides tested for protection against the tick Dermacentor andersoni Stiles on cattle. Proceedings of the B.C. Entomological Society 48: 51-54.

WHITEHEAD, W.E. 1934. Records of some Quebec Mallophaga and Anoplura. Report of the Quebec Society for the Protection of Plants 26: 84-87.

WILKINSON, P.R. 1968. Phenology, behavior, and hostrelations of Dermacentor andersoni Stiles in outdoor "rodentaria," and in nature. Canadian Journal of Zoology 46: 677-689.

1972. Sites of attachment of 'prairie' and 'montane' Dermacentor andersoni (Acarina: Ixodidae) on cattle. Journal of Medical Entomology 9: 133-137.

1981. The proportion of immature stages of the Rocky Mountain wood tick (Dermacentor andersoni) feeding on artificially infested cattle. Journal of the Entomological Society of B.C. 78: 38-42.

1982. The potential for selection of cattle resistant to tick paralysis and attachment of Rocky Mountain wood ticks. Agriculture Canada, Research Highlights 1981 pp. 25-28, Lethbridge, Alberta.

WILKINSON, P.R., AND J.E. LAWSON. 1965. Differences of sites of attachment of Dermacentor andersoni Stiles to cattle in southeastern Alberta and in south central British Columbia, in relation to possible existence of genetically different strains of ticks. Canadian Journal of Zoology 43: 408-411.

WILLSON, P.J. 1982. Giardiasis in two calves. Canadian Veterinary Journal 23: 83.

WITTNICH, C. 1976. Entamoeba histolytica infections in a german shepherd dog. Canadian Veterinary Journal 17: $259-263$.

WOBESER, G.A. 1971. The occurrence of Echinococcus multilocularis (Leuckart, 1863) in cats near Saskatoon, Saskatchewan. Canadian Veterinary Journal 12: 65-68. 
WOO, P., M.A. SOLTYS, AND A.C. GILLICK. 1970.

Trypanosomes in cattle in Southern Ontario. Canadian Journal of Comparative Medicine 34: 142-147.
WRIGHT, B. 1979. Mites, ticks, fleas, and lice in the Nova Scotia Museum and Acadia University Museum collections. Science 29: 185-196.

\section{Supplementary Readings}

ALLEN, J.A., AND A.B. WICK WARE. 1922. A preliminary note on parasites infesting domesticated silver black foxes in Canada. Parasitology 14: 27-28.

ANON. 1931. A note on Parametorchis canadensis (Law) from Ontario mink. Report of the Ontario Veterinary College for 1930: 39 .

1948. Liver fluke control in the Burwash area. Report of the Ontario Veterinary College (for 1947): 49-51.

1950. Survey to ascertain the geographical distribution of the large American liver fluke (Fascioloides magna) in Ontario. Report of the Ontario Veterinary College for 1949: 35.

ARTHUR, D.R., AND K.R. SNOW. 1968. Ixodes pacificus Cooley and Kohls, 1943: Its life-history and occurrence. Parasitology 58: 893-906.

BARON, R.W. AND J. WEINTRAUB. 1987.

Lymphocyte responsiveness in cattle previously infested and uninfested with Hypoderma lineatum (de Vill) and H. bovis (L.) (Diptera: Oestridae). Veterinary Parasitology 24: 285-296.

BATT, H.E. 1926. Internal parasites in foxes. Fur Trade Journal of Canada 3: 7.

BECKLUND, W.W. 1964. Revised check list of internal and external parasites of domestic animals in the United States and possessions and in Canada. American Journal of Veterinary Research 25: 1380-1446.

BOULANGER, P., G.M. RUCKERBAUER, G.L. BANNISTER, R.R. MACKAY, AND N.H. PETER. 1971. Anaplasmosis: control of the first outbreak in Canada by serological identification and slaughter. Canadian Journal of Comparative Medicine 35: 249-257.

BROWN, J.H. 1955. Colorado tick fever in Alberta. Canadian Journal of Zoology 33: 389-390.

BRUCE, E.A. 1920. Information for farmers and ranchers regarding tick paralysis in British Columbia. Dominion of Canada, Department of Agriculture, Bulletin No. $133.7 \mathrm{pp}$.

CAMERON, A.E. 1923. Notes on buffalo: anatomy, pathological conditions, and parasites. The Veterinary Journal 79: 331-336.

1924. Some further notes on buffalo. The Veterinary Journal 80: 413-417.

CAMERON, T.W.M. 1933. The important helminth parasites of stock in the British Empire. British Veterinary Journal 89: 505-514.

1944. The morphology, taxonomy, and life history of Metorchis conjunctus (Cobbold, 1860). Canadian Journal of Research 22: 6-16.
CAMPBELL, D.J. 1960. Parasitic gastro-enteritis of sheep. Canadian Veterinary Journal 1: 151-156.

CHOQUETTE, L.P.E. 1954. Verminous Bronchitis in cattle. Canadian Journal of Comparative Medicine 18: 347-356.

CHURCH, T.L., AND O.M. RADOSTITS. 1981. A retrospective survey of diseases of feedlot cattle in Alberta. Canadian Veterinary Journal 22: 27-30.

COGLEY, T.P., J.R. ANDERSON, AND J.

WEINTRAUB. 1981. Ultrastructure and function of the attachment organ of warble fly eggs (Diptera: Oestridae, Hypodermatinae). International Journal of Insect Morphology and Embryology 10: 7-18.

COSTELLO, R.A. 1986. Control of insects and ticks on livestock. Province of British Columbia, Ministry of Agriculture and Fisheries Publication. 20pp.

CURTICE, C. 1892. Parasites. Being a list of those infesting the domesticated animals and man in the United States. Journal of Comparative and Veterinary Archives (1892): 223-236.

CURTIS, L.C. 1959. Control of the Rocky Mountain wood tick, Dermacentor andersoni Stiles (Acarina: Ixodidae), with ground sprays of Dieldrin and Heptachlor. Proceedings of the B.C. Entomological Society 56: 13-15.

DOHOO, I.R., R.A. CURTIS, AND G.G. FINLEY. 1985. A survey of sheep diseases in Canada. Canadian Journal of Comparative Medicine 49: 239-247.

DUBEY, J.P., AND J.L. MAHRT. 1978. Isospora neorivolta sp. $\mathrm{n}$. from the domestic dog. Journal of Parasiology 64: 1067-1073.

FALLIS, A.M. 1986. Griffith Evans 1835 - 1935. Discoverer of the first pathogenic trypanosome. Canadian Veterinary Journal 27: 336-338.

FAYER, R. 1981. Toxoplasmosis update and public health implications. Canadian Veterinary Journal 22: 344-352.

FRASER, C.M., AND D.J. CAMPBELL. 1966. Variability of resistance of calves to acquisition of infection by Nematodirus helvetianus. Canadian Veterinary Journal 7: 193-198.

FRECHETTE, J.L., AND P. LAMOTHE. 1981. Milk production effect of a morantel tartrate treatment at calving in dairy cows with subclinical parasitism. Canadian Veterinary Journal 22: 252-254.

FRIEND, S.C.E., and P.H.G. STOCKDALE. 1980. Experimental Eimeria bovis infection in calves: a histopathological study. Canadian Journal of Comparative Medicine: 44: 129-140 
FRIMETH, J.P., AND H.P. ARAI. 1984. A survey for the microfilariae of the canine heartworm, Dirofilaria immitis, in the Calgary region of southern Alberta. Canadian Veterinary Journal 25: 14-16.

GADBOIS, P., J.L. FRECHETTE, A. VILLENEUVE, AND B.I. GROVES. 1985. A new approach in the prevention of gastrointestinal parasitic infections in cattle. Canadian Veterinary Journal 26: 127-131.

GAJADHAR, A.A., J.P. CARON, AND J.R. ALLEN. 1985. Cryptosporidiosis in two foals. Canadian Veterinary Journal 26: 132-134.

GOODING, R.H., AND J. WEINTRAUB. 1960. The genitalia of Hypoderma bovis (L.) and H. lineatum (De Vill.) (Diptera: Oestridae). Canadian Journal of Zoology 38: 565-574.

GREGSON, J.D. 1936. Studies on the rate of tick feeding in relation to disease. Proceedings of the B.C.

Entomological Society (1936): 15-21.

1940. The discovery of an ixovotoxin in Dermacentor andersoni eggs. Proceedings of the B.C. Entomological Society (1940): 9-10.

1942a. The coast tick (Ixodes californicus Banks) problem in British Columbia. Canadian Entomologist 74: 3-5.

1942b. Notes on the laboratory rearing of some Canadian ticks. Proceedings of the B.C. Entomological Society (1942): 32-35.

1943. The enigma of tick paralysis. Proceedings of the

B.C. Entomological Society (1943) 40: 19-23.

1951. Notes on the spring activity of the Rocky Mountain wood tick, Dermacentor andersoni Stiles (Acarini: Ixodidae). Proceedings of the B.C. Entomological Society 47: 4-7.

1973. Tick paralysis, an appraisal of natural and experimental data. Canadian Department of Agriculture, Ottawa, Ontario 109pp.

1974. Records of tick paralysis in livestock in British Columbia. Journal of the Entomological Society of British Columbia 63: 13-18.

GREGSON, J.D., AND G.P. HOLLAND. 1944. Devices for charting and obtaining naturally emerged cattle warbles (Diptera: Oestridae). Proceedings of the B.C. Entomological Society (1944) 41: 31-33.

HADWEN, S. 1914. Notes on the life-histories of bloodsucking Diptera of British Columbia, with special reference to the Tabanidae. Proceedings of the B.C. Entomological Society (1914): 46-49.

HEARLE, E. 1938. Insects and allied parasites injurious to livestock and poultry in Canada. Canadian Department of Agriculture Publicate 604 (Farmers Bulletin 53).

HOLLAND, G.P. 1939. Notes on the ecology of Dermacentor andersoni in southern Alberta. Proceedings of the B.C. Entomological Society (1939) 8-11.
ISLER, C.M., J.E.C. BELLAMY, AND G.A.

WOBESER. 1987. Labile neurotoxin in serum of calves with "nervous coccidiosis. Canadian Journal of Veterinary Research 51: 253-260.

1987. Pathogensis of neurological signs associated with bovine enteric coccidiosis. Canadian Journal of Veterinary Research 51: 261-270.

1987. Characteristics of the labile neurotoxin associated with nervous coccidiosis. Canadian Journal of Veterinary Research 51: 271-276.

KENNEDY, A.H. 1950. Paragonimus infection in a ranchraised fox. Report of the Ontario Veterinary College for 1949: 132-137.

KENNEDY, B.J., AND C.I. KIR K. 1984. ELISA testing for heartworm. Canadian Veterinary Journal 25: 406.

KINGSCOTE, A.A. 1930. A case of paragonimiasis in the Ontario domestic fox. Report of the Ontario Veterinary College, p. 40.

1932. A case of Paragonimus kellicotti (lung-fluke) infestation in ranch-raised mink. Report of the Ontario Veterinary College for 1931: 62.

1938. Common parasites of Canadian animals. Canadian Journal of Comparative Medicine 2: 47-55.

1953. Internal parasites of swine. Ontario Department of Agriculture Bulletin No. 496. 36 pp. Toronto, Canada.

LAUTENSLAGER, J.P., AND J.O.D. SLOCOMBE. 1984. Immunodiagnosis of occult heartworm. Canadian Veterinary Journal 25: 407-408.

LAW, R.G. 1930. The mink in captivity. Ontario Department of Game Fish Bulletin 3: 1-23 (see p. 12-18).

LAW, R.G., AND A.C. SECORD. 1931. Control of lung worm in silver foxes. North American Veterinarian 12: $42-44$.

LEVINE, N.D., AND V. IVENS. 1964. Isospora species in the dog. Journal of Parasitology 51: 859-864.

LEVINE, N.D., AND V. IVENS. 1986. The coccidian parasites (Protozoa, Apicomplexa) of Artiodactyla. Illinois Biological Monographs. 55: 265pp.

LINDQUIST, E.E. 1979. 12. Acari pp. 252-290. In H.V. Danks (ed.). Canada and its Insect Fauna. Memoirs of the Entomological Society of Canada 108. 573 pp.

LUBINSKY, G. 1958. On the 12-hooked oncospheres of Canadian strains of Echinococcus. Canadian Journal of Zoology 36: 883-887.

MAIL, G.A. 1942. Tick control with special reference to Dermacentor andersoni Stiles. Canadian Journal of Agriculture Science 23: 59-67.

MARSH, H. 1952. Disease problems in range livestock. Canadian Journal of Comparative Medicine 16: 89-96.

McALLISTER, R.A. 1964. An outbreak of Toxoplasmosis in an Ontario chinchilla herd. Canadian Journal of Comparative Medicine 28: 53-56. 
McCRAW, B.M. 1951. Some aquatic snails of Ontario and their relation to the possible occurrence of trematode diseases: a preliminary report. Report of the Ontario Veterinary College for 1950: 128-139.

McCRAW, B.M., AND J.O.D. SLOCOMBE. 1985. Strongylus equinus: Development and pathological effects in the equine host. Canadian Journal of Comparative Medicine 49: 372-383.

McGREGOR, J.K., AND D.C. MAPLESDEN. 1955. Gastro-intestinal helminthiasis of sheep. A commentary and case report. Canadian Journal of Comparative Medicine 19: 129-131.

MOILLIET, T.K. 1936. A review of tick paralysis in cattle in British Columbia with mites in several cases.

Proceedings of the B.C. Entomological Society (1936): 35-39.

1937. A review of tick paralysis in cattle in British Columbia. Proceedings of the B.C. Entomological Society 33: 35-39.

NELSON, W.A., AND J. WEINTRAUB. 1972. Hypoderma lineatum (De Vill.) (Diptera: Oestridae): invasion of the bovine skin by newly hatched larvae. Journal of Parasitology 58: 614-624.

NIILO, T. 1970. Bovine coccidiosis in Canada. Canadian Veterinary Journal 11: 91-98.

OGWU, D., AND S. NURU. 1981. Transplacental transmission of trypanosomes in animals and man. A review. The Veterinary Bulletin 52: 381-384.

OLIVER, J.H. JR., P.R. WILKINSON, AND G.M. KOHLS. 1972. Observations on hybridization of three species of North American Dermacentor ticks. Journal of Parasitology 58: 380-384.

PARNELL, I.W. 1934. Studies on the bionomics and control of the bursate nematodes of horses and sheep. I. The effect of urine on the eggs and larvae in the feces. Canadian Journal of Research: 532-538.

POLLEY, L. 1986. Strongylid parasites of horses: Experimental ecology of the free-living stages on the Canadian prairie. American Journal of Veterinary Research 47: 1686-1693.

PULLIN, J.W. 1961. A discussion of the common diseases of sheep. Canadian Veterinary Journal 2: 359-368.

RADOSTITS, O.M., AND P.H.G. STOCKDALE. 1980. A brief review of bovine coccidiosis in western Canada. Canadian Veterinary Journal 21: 227-230.

RAPSEY, G.H., AND R.G. LAW. 1928. Liver fluke in Mink. The Black Fox Magazine 12: 10.

SCHILLHORN VAN VEEN, T.W. 1982. Fasciola cercariae in Quebec. Canadian Veterinary Journal 23: 163.

SLOCOMBE, J.O.D. 1978. Heartworm in dogs in Canada in 1977. Canadian Veterinary Journal 19: 244-247.

SLOCOMBE, J.O.D., B.M. McCRAW, P.W. PENNOCK, N. DUCHARME, AND J.D. BAIRD. 1987. Strongylus vulgaris in the tunica media of arteries of ponies and treatment with ivermectin. Canadian Journal of Veterinary Research 51: 232-235.

SLOCOMBE, J.O.D., AND I. McMILLAN. 1979.

Heartworm in dogs in Canada in 1978. Canadian Veterinary Journal 20: 284-289.

1980. Heartworm in dogs in Canada in 1979. Canadian Veterinary Journal 21: 159-161.

1981. Heartworm in dogs in Canada in 1980. Canadian Veterinary Journal 22: 201-203.

1982. Heartworm in dogs in Canada in 1981. Canadian Veterinary Journal 23: 219-221.

1983. Heartworm in dogs in Canada in 1982. Canadian Veterinary Journal 24: 227-229.

1984. Heartworm in dogs in Canada in 1983. Canadian Veterinary Journal 25: 347-350.

1985. Heartworm in dog in Canada in 1984. Canadian Veterinary Journal 26: 323-327.

1986. Heartworm in dogs in Canada in 1985. Canadian Veterinary Journal 27: 324-328

1986. Heartworm in dogs in western Canada in 1985. American Veterinary Medicine Newsletter, April 7 pp 1-3.

1987. Heartworm in dogs in Canada in 1986. Canadian Veterinary Journal 28: 491-495.

SMITH, H.J. 1987. Factors affecting preconditioning of Trichinella spiralis nativa larvae in musculature to low temperatures. Canadian Journal of Veterinary Research 51: 169-173.

1987. Evaluation of the ELISA for the serological diagnosis of trichinosis in swine. Canadian Journal of Veterinary Research 51: 194-197.

SMITH, H.J., AND K.E. SNOWDEN. 1987. Detection of Trichinella spiralis nativa antibodies in porcine sera by ELISA using $T$. spiralis spiralis excretory-secretory antigen. Canadian Journal of Veterinary Research 51: 413-414.

SPENCER, G.J. 1952. The 1951 status knowledge of the insects of British Columbia. Proceedings of the B.C. Entomological Society 48: 36-41.

1958. On the reproductive potential of the sheep nostril fly Oestrus ovis L. (Diptera: Oestridae). Proceedings of the B.C. Entomological Society 55: 25-26.

STOCKDALE, P.H.G. 1977. The pathogenesis of the lesions produced by Eimeria zuernii in calves. Canadian Journal of Comparative Medicine 41: 338-344.

STOCKDALE, P.H.G., A.R. BAINBOROUGH, C.B. BAILEY, and L. NIILO. 1981. Some pathophysiological changes associated with infection of Eimeria zuernii in calves. Canadian Journal of Comparative Medicine 45: 34-37. 
STOCKDALE, P.H.G., AND T.J. HULLAND. 1970. The pathogenesis, route of migration and development of Crenosoma vulpis in the dog. Pathologia Veterinaria 7: $28-42$.

STOCKDALE, P.H.G., and L. NIILO. 1976. Production of bovine coccidiosis with Eimeria zuernii. Canadian Veterinary Journal 17: 35-37.

STOCKDALE, P.H.G., AND M.E. SMART. 1975. Treatment of crenosomiasis in dogs. Research in Veterinary Science 18: 178-181.

STOGDALE, L. 1984. ELISA testing for occult heartworm infection. Canadian Veterinary Journal 25: 406-407.

1984. Testing for occult heartworm infection. Canadian Veterinary Journal 25: 408-409.

STRICKLAND, E.H. 1949. Wohlfahrtia (Diptera, Metopiidae) myiasis of mink in Alberta. Canadian Entomologist 81: 58-60.

SWALES, W.E. 1939. Notes on the diagnosis and treatment of parasitic diseases of sheep in Canada. Canadian Journal of Comparative Medicine 3: 341-344.

1940. Further experiments on the use of phenothiazine as an anthelmintic for sheep. Canadian Journal of Research 18: 266-271.

SWEATMAN, G.K., AND T.C. HENSHALL. 1962. The comparative biology and morphology of Taenia ovis and Taenia krabbei, with observations on the development of $T$. ovis in domestic animals. Canadian Journal of Zoology 40: 1287-1311.

TAILYOUR, J.M., AND M.J. HAMPTON. 1954. A check on the incidence of trichinosis in swine on six piggeries in British Columbia. Canadian Journal of Comparative Medicine 18: 311-312.

TRYPHONAS, L., Z. ZAWIDZKA, M.A. BERNARD, AND E.A. JANZEN. 1977. Visceral Leishmaniasis in a dog: clinical, hematological, and pathological observations. Canadian Journal of Comparative Medicine 41: 1-12.
TYRRELL, J.B. 1883. On the occurrence in Canada of two species of parasitic mites. Proceedings of the Canadian Institute 1: 332-342.

VIENS, P. La "larva migrans viscerale" a Montreal ou le somet de l'iceberg Bordeaux Medical 10: 697-698.

WEBSTER, G.A. 1958. A report on Toxocara canis Werner, 1782. Canadian Journal of Comparative Medicine 22: 272-279.

WHITNEY, H., A. VILLENEUVE, J.L. FRECHETTE, AND B. PINEL-ALLOUL. 1981. Intermediary hosts for Fasciola hepatica identified in Quebec. Canadian Veterinary Journal 22: 334.

WICKWARE, A.B. 1923. Parasites of the silver-black fox. Canadian Veterinary Record 4: 128-129.

WILKINSON, P.R. 1967. The distribution of Dermacentor ticks in Canada in relation to bioclimatic zones. Canadian Journal of Zoology 45: 517-537.

WILLSON, P.J., and S.D. ACRES. 1982. A comparison of dichromate solution floatation and fecal smears for diagnosis of cryptosporidiosis in calves. Canadian Veterinary Journal 23: 240-246.

WOBESER, G., A.A. GAJADHAR, AND H.M. HUNT. 1985. Fascioloides magna: occurrence in Saskatchewan and distribution in Canada. Canadian Veterinary Journal 26: 241-244.

WOOLFGANG, R.W., AND J.B. POOLE. 1956. Distribution of Echinococcus disease in Northwestern Canada. American Journal of Tropical Medicine and Hygiene 5: 869-871.

YANG, Y.H., AND H.C. GRICE. 1964. Klossiella muris parasitism in laboratory mice. Canadian Journal of Comparative Medicine 28: 63-66.

YANG, J., J.S. KEYSTONE, L. McINTYRE, AND H. SPENCE. 1982. Toxocara antibodies in veterinary personnel. Canadian Veterinary Journal 23: 126-128.

ZAUGG, J.L., AND K.L. KUTTLER. 1987. Experimental infectins of Babesia bigemina in American bison. Journal of Wildlife Diseases 23: 99-102. 
Artiodactyla 31

Bos taurus 31

Bovidae 31

Canidae $\quad 33$

Canis familiaris 33

Caprahircus $\quad 32$

Carnivora 33

Cat 34

Dog 33

Equidae $\quad 35$

Equus caballus

Eutheria 31

35

\section{Parasite Index}

Acanthocephala $\quad 23$

Acari 23

Acaridida 23

Acariformes 23

Actinedida 24

Adeleina 5

Adenophorea 12

Aelurostrongylus spp. $\quad 17$

Alaria arisaemoides 9

Alaria canis 9

Alaria spp. 9

Alfortia edentatus see Strongylus edentatus $\quad 28$

Alfortia spp.

see Strongylus spp. $\quad 20$

Amoebida 8

Ancylostomatidae $\quad 17$

Ancylostomatoidea $\quad 17$

Ancylostomum caninum

Ancylostomum spp. $\quad 17$

Angiostrongylidae $\quad 17$

Animalia 5

Anoplocephala magna 10

Anoplocephala mamillana

see Paranoplocephala mamillana 10

Anoplocephala perfoliata

Anoplocephala spp. $\quad 10$

Anoplocephalidae $\quad 10$

Apicomplexa 5

Arachnida 23

Archiacanthocephala

Argasidae $\quad 24$

Ascaridida 13

Ascarididae 13

Ascaridoidea 13

Ascaris lumbricoides

Ascaris suum 13

Ascaris spp. 13

Ascarops spp. 16

Atractidae $\quad 14$
Felidae $\quad 34$

Felis catus $\quad 34$

Goat 32

Horse 35

Mammalia 31

Ovis aries $\quad 32$

Ox 31

Perissodactyla $\quad 35$

Pig 33

Sheep 32

Suidae $\quad 33$

Sus scrofa 33

Balantidium spp. 7

Belascaris mystax see Toxocara cati 14

Bovicola bovis see Damalinia bovis

Bovicola caprae see Damalinia caprae 27

Bunostomum phlebotomum

Bunostomum trigonocephalum

Bunostomum spp. $\quad 17$

Calicophoron microbothrioides

9

Calliphora spp. 26

Calliphoridae 26

Capillaria aerophila 12

Capillaria hepatica 12

Capillaria longipes 13

Capillaria spp. $\quad 13$

Ceratophyllidae $\quad 27$

Cestoidea 10

Chabertia ovina 18

Chabertia spp. $\quad 18$

Chabertiidae $\quad 18$

Chelicerata 23

Cheyletidae 24

Cheyletiella spp. 24

Chorioptes bovis 23

Ciliophora 7

Citellinema bifurcatum $\quad 21$

Cooperia curticei 21

Cooperia mcmasteri 22

Cooperia oncophora 22

Cooperia punctata $\quad 22$

Cooperia spp. 22

Corynosoma semerme 23

Cosmocercoidea 14

Crenosoma vulpis 17

Crenosomatidae $\quad 17$

Cryptocotyle lingua 9

Cryptosporidium spp. 5

Ctenocephalides canis $\quad 27$ 
Ctenocephalides felis 27

Ctenocephalides felis felis

Ctenocephalides spp. 28

Cuterebra spp. 26

Cuterebridae 26

Cyathostoma spp. 20

Cyathostomum catinatum

Cyathostomum coronatum 19

Cyathostomum labiatum 19

Cyathostomum labratum $\quad 19$

Cyclophyllidea $\quad 10$

Cylicocyclus elongatus $\quad 19$

Cylicocyclus insigne 19

Cylicocyclus nassatus $\quad 19$

Cylicocyclus radiatus $\quad 19$

Cylicodontophorus bicornatus

Cylicostephanus calicatus

Cylicostephanus goldi 19

Cylicostephanus poculatus

Cylicostomum bicornatum

see Cylicodontophorus bicornatus

Cylicostomum calicatum see Cylicostephanus calicatus 19

Cylicostomum catinatum see Cyathostomum catinatum

Cylicostomum coronatum see Cyathostomum coronatum

Cylicostomum elongatum see Cylicocyclus elongatus 19

Cylicostomum goldi see Cylicostephanus goldi 19

Cylicostomum insigne see Cylicocyclus insigne 19

Cylicostomum labiatum see Cyathostomum labiatum

Cylicostomum labratum see Cyathostomum labratum

Cylicostomum nassatum see Cylicocyclus nassatus 19

Cylicostomum poculatum see Cylicostephanus poculatus

Cylicostomum radiatum see Cylicocyclus radiatus 19

Cysticercus bovis see Taeniarhyncus saginatus

Cysticercus cellulosae see Taenia solium 11

Cysticercus ovis see Taenia ovis 11

Cysticercus tenuicollis see Taenia hydatigena

Damalinia bovis 27

Damalinia caprae 27

Damalinia equi 27

Damalinia limbata 27

Damalinia spp. 27

Delafondia vulgaris see Strongylus vulgaris 20

Demodex bovis 24

Demodex phylloides

Demodex spp. 24
Demodicidae 24

Dermacentor albipictus 25

Dermacentor andersoni 25

Dermacentor erraticus var. albipictus see Dermacentor albipictus 25

Dermacentor variabilis

25

Dermacentor venustus see Dermacentor andersoni 25

Dermacentor spp. 25

Dermanyssidae 24

Dicrocoeliidae 9

Dicrocoelium dendriticum

Dicrocoelium lanceolatum see Dicrocoelium dendriticum

Dictyocaulidae 21

Dictyocaulus arnfieldi $\quad 21$

Dictyocaulus filaria 21

Dictyocaulus viviparus

Dictyocaulus spp. 21

Digenea 9

Dilepididae 11

Dioctophyma renale 12

Dioctophymatidae $\quad 12$

Dioctophymatoidea 12

Dipetalonema spp. $\quad 15$

Diphyllobothriidae 12

Diphyllobothrium dendriticum

Diphyllobothrium latum

Diphyllobothrium spp.

12

Diplomonadida 7

Diplostomidae 9

Diptera 26

Dipylidium caninum

Dipylidium spp.

Dirofilaria immitis

Dirofilaria spp. $\quad 15$

Dracunculidae 15

Dracunculoidea 15

Dracunculus insignis

Echinococcus granulosus

Echinococcus multilocularis

11

Echinococcus spp.

Eimeria ahsata 5

Eimeria alabamensis 5

Eimeria arloingi 5

Eimeria aubernensis 5

Eimeria bovis 5

Eimeria bukidnonensis 5

Eimeria canadensis 5

Eimeria canis 5

Eimeria crandallis 5

Eimeria cylindrica 5

Eimeria deblieki 5

Eimeria ellipsoidalis 5

Eimeria faurei 5

Eimeria granulosa 6

Eimeria illinoisensis 6

Eimeria intricata 6

Eimeria leuckarti 6

Eimeria ninakohlyakimovae

Eimeria ovina 6 
Eimeria pallida 6

Eimeria parva 6

Eimeria punctata 6

Eimeria wyomingensis

Eimeria zuernii 6

Eimeria spp. 6

Eimeriina 5

Endolimax nana 8

Enoplida 12

Entamoebablattae 8

Entamoeba coli 8

Entamoeba histolytica 8

Eucestoda 10

Eucoccidiida 5

Eumetazoa 9

Fasciola hepatica 9

Fasciolidae 9

Fascioloides magna 9

Felicola subrostratus $\quad 27$

Filarioidea 15

Filaroides milksi 17

Filaroides spp. 17

Filariidae 15

Filaroididae $\quad 17$

Gamasida 24

Gasterophilidae 26

Gasterophilus intestinalis $\quad 26$

Gasterophilus nasalis 26

Gasterophilus spp.

Giardia canis 7

Giardia spp. 7

Gongylonema pulchrum 16

Gongylonematidae $\quad 16$

Gyalocephalus capitatus $\quad 19$

Gyalocephalus spp. 19

Habronema majus 16

Habronema microstoma see Habronema majus

Habronema muscae 16

Habronema spp. 16

Habronematidae $\quad 16$

Habronematoidea 16

Haemaphysalis cinnabarina 25

Haemaphysalis leporis-palustris 25

Haematopinidae 28

Haematopinus asini 28

Haematopinus eurysternus

Haematopinus suis $\quad 28$

Haematopinus spp. $\quad 28$

Haemonchus contortus

Haemonchus spp. 22

Heligmosomidae 21

Heterophyidae 9

Hexamitidae 7

Hippobosidae 26

Hyostrongylus rubidus 22

Hyostrongylus spp. 22

Hypoderma bovis 26

Hypoderma lineatum
Hypoderma spp. $\quad 26$

Hystrichopsyllidae 27

Insecta 26

Ischnocera 27

Isospora bigemina 6

Isospora canis 6

Isospora felis 6

Isospora ohioensis 6

Isospora rivolta 6

Isospora suis 6

Isospora wallacei 6

Isospora spp. 7

Ixodes cookei 25

Ixodes hexagonus var. cookei see Ixodes cookei 25

Ixodes ricinus 25

Ixodes ricinus scapularis $\quad 25$

Ixodes spp. 25

Ixodida 24

Ixodidae 25

Kinetofragminophorea 7

Kinetoplastida 7

Klossiella equi 5

Linognathidae 28

Linognathus pedalis 28

Linognathus piliferus

see Linognathus setosus 28

Linognathus setosus $\quad 28$

Linognathus stenopsis $\quad 28$

Linognathus vituli 28

Linognathus spp. $\quad 28$

Lobosea 8

Lucilia illustris 26

Lucilia sericata $\quad 26$

Macracanthorhynchus hirudinaceus 23

Mallophaga 27

Mastigophora 7

Melophagus ovinus 26

Mesocestoides spp. 11

Mesocestoididae 11

Metastrongylidae $\quad 18$

Metastrongyloidea $\quad 17$

Metastrongylus apri $\quad 18$

Metastrongylus elongatus see Metastrongylus salmi 18

Metastrongylus pudendotectus 18

Metastrongylus salmi 18

Metastrongylus spp. $\quad 18$

Metorchis conjunctus 9

Metorchis spp. 9

Molineidae 21

Moniezia benedeni 10

Moniezia expansa

Moniezia spp. $\quad 10$

Monodontus phlebotomus see Bunostomum phlebotomum 17

Monodontus trigonocephalus

see Bunostomum trigonocephalum 17 
Monodontus spp.

see Bunostomum spp.

Monopsyllus vison 27

Muellerius capillaris $\quad 18$

Muellerius spp. 18

Multiceps spp. 11

Nanophyetidae $\quad 9$

Nanophyetus salmincola 9

Nematoda 12

Nematodirus abnormalis $\quad 21$

Nematodirus battus 21

Nematodirus filicollis 21

Nematodirus helvetianus

Nematodirus spathiger

Nematodirus spp. $\quad 21$

Notoedres spp. $\quad 24$

Oesophagodontus robustus

20

Oesophagodontus spp. 20

Oesophagostomum columbianum

Oesophagostomum dentatum 18

Oesophagostomum quadrispinulatum 18

Oesophagostomum radiatum

Oesophagostomum venulosum

Oesophagostomum spp. $\quad 18$

Oestridae 26

Oestrus ovis 27

Oligacanthorhynchida $\quad 23$

Oligacanthorhynchidae $\quad 23$

Ollulanus tricuspis 21

Onchocerca cervicalis 15

Onchocerca gutturosa 16

Onchocerca lienalis 16

Onchocerca spp. 16

Onchocercidae 15

Opisthorchiidae 9

Oslerus osleri 17

Ostertagia circumcincta

Ostertagia lyrata 22

Ostertagia ostertagi 22

Ostertagia trifurcata 22

Ostertagia spp. 23

Otobius lagophilus 24

Otobius megnini 25

Otobius spp. 25

Otodectes cynotis 24

Otodectes cynotis canis 24

Otodectes cynotis felis

Oxyurida 14

Oxyuridae 14

Oxyuris equi 14

Oxyuris spp. $\quad 14$

Oxyuroidea 14

Palaeacanthocephala $\quad 23$

Parafilaria bovicola 15

Paragonimus kellicotti $\quad 10$

Paragonimus spp. $\quad 10$

Parametorchis complexus 9

Parametorchis manitobensis see Parametorchis complexus 9

Paramphistomidae 9
Paramphistomum liorchis $\quad 10$

Paramphistomum microbothrioides see Calicophoron microbothrioides

Paramphistomum spp. $\quad 10$

Paranoplocephala mamillana 10

Parascaris equorum 13

Parascaris spp. $\quad 14$

Parasitiformes 24

Parelaphostrongylus tenuis

Pelodera strongyloides $\quad 14$

Petrovinema poculatum see Cylicostephanus poculatus

Phaenicia sericata see Lucilia sericata 26

Phormia regina 26

Phormia terranovae 26

Physaloptera rara 16

Physalopteridae $\quad 16$

Physalopteroidea $\quad 16$

Physocephalus spp. 16

Plagiorchiidae $\quad 10$

Plagiorchis massino $\quad 10$

Platyhelminthes 9

Pneumonyssus spp. $\quad 24$

Pneumostrongylus tenuis see Parelaphostrongylus tenuis 18

Polymorphida 23

Polymorphidae 23

Poteriostomum spp. $\quad 20$

Probstmayria vivipara : 14

Protophormia terranovae see Phormia terranovae 26

Protostrongylidae $\quad 18$

Protostrongylus spp. $\quad 18$

Protozoa 5

Pseudophyllidea 12

Psorergates bos 24

Psorergatidae 24

Psoroptes spp. 24

Psoroptidae 23

Pulicidae 27

Rhabditida $\quad 14$

Rhabditidae $\quad 14$

Rhabditis strongyloides see Pelodera strongyloides 14

Rhabditoidea 14

Rhipicephalus sanguineus $\quad 25$

Rhipicephalus spp. 25

Sarcocystis cruzi 7

Sarcocystis hirsuta 7

Sarcocystis spp.

Sarcodina 8

Sarcomastigophora 7

Sarcoptes scabiei 24

Sarcoptidae 24

Secernentea 13

Setaria equina 16

Setaria labiatopapillosa $\quad 16$

Setaria spp. $\quad 16$

Siphonaptera 27 
Siphunculata 28

Skrjabinema spp. $\quad 14$

Solenopotes capillatus

Spirocerca spp. $\quad 16$

Spirocercidae 16

Spirurida 15

Spiruroidea 16

Sporozoea 5

Stephanofilaria stilesi 15

Stephanurus dentatus $\quad 20$

Strongylida 17

Strongylidae $\quad 19$

Strongyloidea 18

Strongyloides papillosus

Strongyloides ransomi

Strongyloides stercoralis

Strongyloides spp. 15

Strongyloididae 15

Strongylus edentatus 20

Strongylus equinus 20

Strongylus vulgaris 20

Strongylus spp. 20

Syngamidae 20

Taenia crassiceps $\quad 11$

Taenia elliptica 11

Taenia hydatigena 11

Taenia ovis 11

Taenia pisiformis

Taenia serialis

Taenia solium 11

Taenia taeniaeformis 11

Taenia spp. $\quad 12$

Taeniarhyncus saginatus $\quad 12$

Taeniidae 11

Tamiophila grandis

Telodorsagia davtiani

Thelazia gulosa 16

Thelazia lacrymalis $\quad 16$

Thelazia skrjabini 16

Thelazia spp. $\quad 17$

Thelaziidae $\quad 16$

Thelazioidea 16

Thysanosoma actinioides

Thysanosoma spp. $\quad 10$

Toxascaris leonina 14

Toxascaris limbata see Toxascaris leonina

Toxascaris spp. $\quad 14$

Toxocara canis 14

Toxocara cati 14

Toxocara mystax see Toxocara cati

Toxocara spp. $\quad 14$

Toxoplasma gondii
Toxoplasma spp. $\quad 7$

$\begin{array}{lcl}\text { Trematoda } \quad 9 & \\ \text { Trichinella spiralis } & 12\end{array}$

Trichinella spiralis spiralis

Trichinella spp. $\quad 12$

Trichinellidae $\quad 12$

Trichinelloidea 12

Trichodectes canis $\quad 27$

Trichodectes equi 27

Trichodectes spp. 27

Trichomonadida 8

Trichomonas spp. $\quad 8$

Trichonema catinatum

see Cyathostomum catinatum 19

Trichostomatida 7

Trichostrongylidae $\quad 21$

Trichostrongyloidea $\quad 21$

Trichostrongylus axei 23

Trichostrongylus colubriformis

Trichostrongylus extenuatus see Trichostrongylus axei 23

Trichostrongylus vitrinus 23

Trichostrongylus spp. 23

Trichuridae 12

Trichuris discolor

Trichuris ovis 13

Trichuris suis 13

Trichuris vulpis 13

Trichuris spp. $\quad 13$

Triodontophorus brevicauda $\quad 20$

Triodontophorus intermedius see Triodontophorus serratus

Triodontophorus minor $\quad 20$

Triodontophorus serratus 20

Triodontophorus tenuicollis $\quad 20$

Triodontophorus spp. 20

Troglotrematidae $\quad 10$

Trombiculidae 24

Trypanosoma equiperdum 7

Trypanosoma rutherfordi 7

Trypanosoma theileri 7

Trypanosoma uniforme 8

Trypanosoma spp. $\quad 8$

Uncinaria stenocephala $\quad 17$

Uncinaria spp. $\quad 17$

Uniramia 26

Warrenius bifurcatus see Citellinema bifurcatum 21

Wohlfahrtia opaca 26

Wohlfahrtia vigil 26

Zoomastigophorea $\quad 7$ 



